

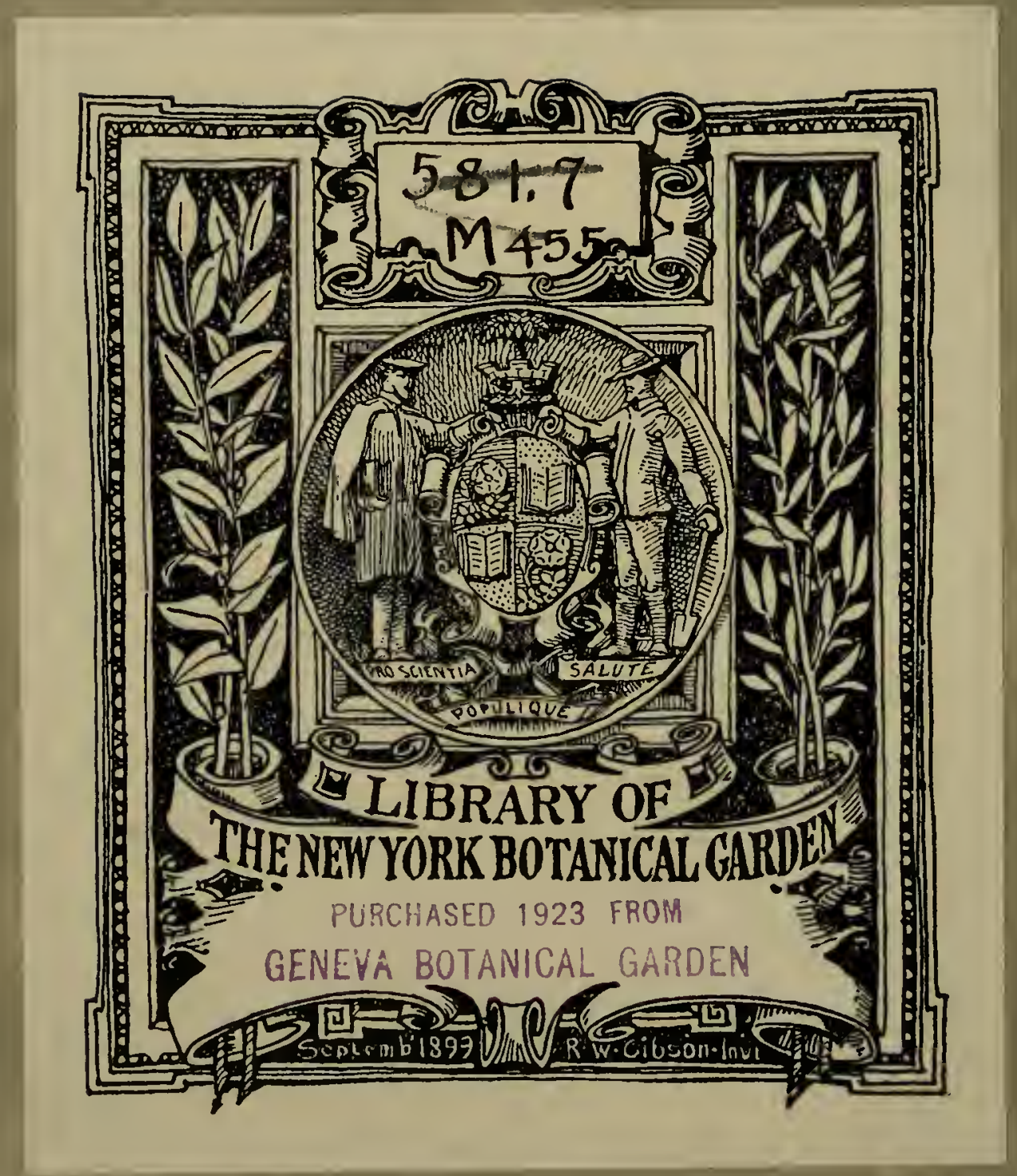






\section{UNIVERSITÉ DE GENĖVE}

\section{ÉTUDES}

\section{D'ANATOMIE COMPAREE}

SUR LES

\section{ORGANES DE VÉGÉTATION \\ DANS LE GENRE CAREX}

THËSE

PRÉSENTÉE A LA FACULTÉ DES SCIENCES POUR L'OBTENTION DU GRADE DE DOCTEUR ÉS-SCIENCES NATURELLES

$$
\begin{aligned}
& \text { PAR } \\
& \text { ANTOINE MAZEL LISAPAR: } \\
& \text { DE GENEYE } \\
& \text { aven } 7 \text { feanches WARDEN }
\end{aligned}
$$

MÉMOIRE COURONNÉ PAR LA FACULTÉ DES SCIENCES

(PRIX DAVY)

\section{GENÈVE}

IMPRIMERIE SOULLIER, RUE DE LA CITÉ, 19

1891 
-636
1439 


\section{INTRODUCTION}

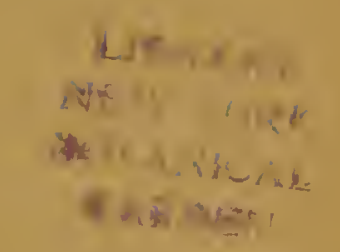

Parmi les nombreuses plantes que nous sommes appelés à rencontrer sur notre chemin, il en est que leur taille morleste, leur peu d'apparence, les. abords souvent difficiles de leurs stations et la difficulté de leur détermination, font volontiers laisser de côté. A cette catégorie appartiennent certainement les Carex et leur congénères.

Et pourtant rien n'est moins mérité que ce dédain-là, car si l'extérieur n'a pas de dehors alléchants pour l'œil, il en est tout autrement lorsque celui-ci s'arme des secours du microscope, et qu'aidé d'un si puissant auxiliaire, il pénètre avec lui dans l'organisation intime de ces végétaux.

En effet, il se trouve là au milieu d'un contingent de faits aussi intéressants sous le rapport de la systématique que sous celui de la physiologie et de l'anatomie végétales.

Le genre Carex, et probablement aussi toute la famille à laquelle il appartient, forme un groupe de plantes admirablement constituées pour une étude d'anatomie ou de physiologie; il en est peu, parmi nos végétaux, qui présentent un système d'adaptations mécaniques, de protections naturelles contre les agents extérieurs plus complet et plus intéressant. 
Aussi, bien que parti avec l'idée de faire une monographie des Cypéracées en général, et cela surtout au point de vue systématique, nous nous sommes laissé entrainer par tant de recherches et d'études intéressantes à n'étudier' que le genre Carex, en détail et au point de vue de l'anatomie seulement, nous réservant de revenir un jour plus spécialernent et en meilleure connaissance de cause, à des recherches ayant pour but la systématique pure.

Qu'il nous soit permis, avant d'aller plus loin, de remercier bien sincèrement notre ancien professeur, Monsieur le Dr J. Müller, de l'ilée qu'il nous a donnée en nous proposant comme thèse les Cypéracées; qu'il veuille bien aussi nous pardonner, si nous nous sommes un peu écarlé du chemin qu'il avait tracé. Il voudra bien comprendre que les circonstances ont été plus fortes que notre volonté. Ce travail, ébauché à Genève, a été presque terminé en Allemagne dans les laboratoires de l'Instilut botanique de Tubingue, sous l'aimable direction de notre savant professeur, le $\mathrm{D}^{\mathrm{r}} \mathrm{II}$. Vöchting. C'est pour nous un devoir agréable entre tous, de lui témoigner ici toute notre reconnaissance.

- Ce fut lui qui nous persuada que, pour mener à bien un travail de systématique aussi considérable, il fallait d'abord posséder son sujet au point de vue de l'anatomie; ce ful lui encore qui conseilla à l'auteur de ce mémoire de le restreindre à l'étude anatomique des organes de végétation.

Il y aurait encore d'autres per'sonnes à remercier ici et qui toutes se sont intéressées d'une laçon ou d'une autre à nos recherches: ce sont d'abord les deux assistants de l'Institut botanique de Tubingue, MM. les docteurs Zimmermann et Rosen, M. le professeur A. de Candolle, qui a bien voulu mettre sa riche bibliothèque à notre disposition, Monsieur le professeur Brun, à qui nous 
devons l'arantage d'aroir pu nous servir de ses excellents instruments, Monsieur le professur Dr Chodat, pour ses nombreux et bons conseils, et Monsieur le professeur Thurý, pour l'intérêt qu'il a pris au succès de ce travail.

Nous savons fort bien que notre : œurre n'est pas la perfection; ce n'est pas après un premier essai qu'on peut prétendre arriver à quelque chose de très bien et de très complet. De tels sujets, surtout quand on n'en a pas l'habitude, sont hérissés de difficultés; aussi n'estce pas sans hésitation que nous avons abordé celte étude; et quelque flatteurs qu'aient été les encourag̨ements, nous nous sommes bien souvenl demandé dans le cours de ce travail, s'il n'était point téméraire de l'entreprendre, et si nous n'aurions pas dù en laisser le soin à de plus capables. Mais la pensée que même arec ses inperfections et ses lacunes, il ne serait peut être point inutile, que sous plus d'un point de vue, il ferait faire plus ample connaissance arec le genre Carex, et que les laits qui y sont conslatés pouriaient servir un jour ou l'autre de point de départ pour des travaux plus complets, celte pensée, disons-nous, le sentiment d'avoir travaillé par nous-mêmes, et d'aroir trouvé ce que nous avons trouvé par nos seuls moyens, a levé nos scrupules et nous a donné le courage de présenter ce travail à la Faculté des Sciences de l'Université.

Cette étude du genre Carex a été divisée en deux parties; dans la première il sera question des organes de végétation à un point de vue général; la seconde comprendra les analyses microscopiques détaillées de plus de quarante espèces, la plupart indigènes et dont quelques-unes seulement sont étrangères à la Suisse. Cette partie analytique aura sans doute quelque actualité, puisque l'anatomie a, de nos jours, une tendance toujours plus grande à nous 
venir en aide dans les difficultés qui surgissent à chaque pas dans la détermination des espèces voisines.

Plusieurs tentatives dans ce sens ont été déjà faites et nous pouvons citer à ce propos quelques travaux tels que celui du Dr Chrisl: Sur quelques espèces du genre Carex. (Bulletin des travaux de la Société Botanique de Genève $n^{0} 5$ ) et quelques autres d'un intèrèt moins direct.

Ce n'est pourtant pas là, la seule littérature contemporaine que l'on possède sur cette tribu. De nos jours ont paru quelques autres mémoires importants, mais qui ne traitent pour la plupart que d'un organe isolé: ce sont entre autres: $10 \mathrm{du}$ Dr Schwendener, die Spaltöffuungen der Gramineen et Cypéraceen (dans les Sitzungsberichte der königlich preussischen Aliademie der Wissenschaften zu Berlin) où l'auteur passe en revue les différentes formes que présente l'appareil stomatique chez les Carex, $2^{\circ}$ de J. Klinge (dans les Mémoires de l'Académie Impériale des Sciences de Saint-Pétersbourg), Vergleichend histologische Untersuchung der Gramineen und Cypéraceen Wurzeln, puis quelques pages isolées dans les auteurs cités à la fin de ce travail.

Quant à nos recherches, elles ont été faites sur environ une cinquantaine d'espèces, provenant soit de nos lierborisations particulières, soit de sujets cultivés aux jardins hotaniques de Genève et de Tubingue, soit sur des échantillons d'herbier qu'on a bien voulu mettre à notre disposition. 


\section{PARTIE GÉNÉRALE}

\section{Etude de la feuille}

Nous verrons que dans cet organe - comme aussi dans la racine, la tige et le rhizôme - nous avons un plan fondamental qui se trouve presque partout, dans chaque espèce, el dont la nature semble ne s'être pas écartée beaucoup.

Ce plan peut être en quelque sorte schématisé pour chacun de ces organes, sans que l'on coure le risque de commettre une erreur trop considérable.

Quiconque s'est occupé un peu de Botanique reconnaît facilement un Carex à son habitus tout particulier, à ses feuilles plus ou moins étroites, linéaires, plus ou moins planes, souvent hérissées d'aspérités, terminées en pointe effilée, presque jamais velues, ou si c'est le cas, pourvues d'un faible duvet, surtout sur la page supérieure et à l'endroit où les gaines foliaires s'entrourrent pour laisser passer la tige. Mais le fait est toujours très rar'e, puisque sur toutes les espèces que nous avons analysées microscopiquement, nous n'en avons trouvé qu'une seule, C. hirta, qui présentàt des poils nettement constitués et très visibles dẻjà à l'œil nu. 
Nous verrons dans la suite que pour ce qui est des papilles, il n'en est plus du tout de même.

Si chez les Carex, le plan général d'un organe est facilement reconnaissable au premier coup d'œil, il n'est pas cependant sans piésenter de notables divergences dans les différentes espèces. C'est ainsi que pour la feuille, nous trouverons une grande variété de formes dans les cellules épidermiques, dans les éléments mécaniques, dans l'appareil stomatique, la structure et la disposition des faisceaux, la nature ou le rôle de certaines cellules. Toute espèce pourra, par exemple, présenter un épiderme formé de cellules égales en volume sur les deux pages de la feuille, telle autre présentera par contre, sur la page supérieure, un épiderme formé de grandes cellules presque rondes et plusieurs fois plus grandes que les éléments correspondants de la page inférieure, qui sont restés petits et allongés dañs le sens du plan de la feuille.

Ces différences se trouvent parfois avoir lieu entre des espèces très voisines, et elles sont si marquées souvent que la détermination par l'analyse microscopique devient beaucoup plus aisée et partant plus rapide que celle où l'on se base sur les caractères floraux, opération toujours plus ou moins compliquée.

Nous aurons donc à considérer pour notre étude détaillée de la feuille les différentes parties suivantes: l'épiderme, y compris les stomates el les cellules dites " de plissement 》 (Einrollungsmechanismus), les faisceaux, le tissu sclérenchymateux et le tissu assimilateur.

Mais auparavant résumons en quelques mots l'aspect extérieur de l'organe foliaire dans le genre Carex.

En général nous avons, comme il l'a été dit plus haut, la forme étroite et linéaire, propre aux feuilles de Monocotylédones, avec nẹrvures courant parallèlement et s'anas- 
tomosant ici et là entre elles. Ces nervures deviennent très évidentes et peuvent facilement s'étudier, lorsqu'on laisse séjourner pendant quelques jour's une feuille dans de l'eau à laquelle on a ajouté quelques gouttes de fuchsine et que l'on décolore ensuite par l'ammoniaque.

D'entre ces nervures, il en est trois qui se font remarquer par leur épaisseur et par le fait qu'elles correspondent toutes trois à un pli de la feuille.

Ces plis sont surtout visibles chez les grandes espèces, telles que C. riparia, C. provincialis, G. maxima, etc.

Nous verrons plus loin que c'est à la nervure médiane que correspondent les cellules de plissement et qu'elles ont une relation étroite avec les phénomènes de turges. cence.

On peut se demander de quelle utilité est pour la feuille la présence de ces trois plis. Il nous semble que celte question trouve sa réponse dans le fait que nous avons affaire ici à une disposition toute mécanique; tout le monde sait en effet qu'une surface très mince et plane présente une résistance beaucoup plus faible aux forces extérieures qu'une autre surface de même épaisseur, mais qui présente une ou plusieurs plicatures, par lesquelles elle acquiert un pouvoir résistant plus grand et par conséquent une plus grande solidité.

Par contre, il existe de nombreuses espèces où la feuille n’étant plus si large ni si mince, n'a plus besoin de cette distribution mécanique. Les revêtements sclérenchymateux des faisceaux suffisent alors pour assurer à l'organe la solidité nécessaire. Pour donner un exemple d'un cas semblable, je citerai celui de C. Davalliana, où nous avons une feuille presque triangulaire dont les angles sont pourvus de groupes sclérenchymateux et où les faisceaux, à pari celui qui correspond à la nervure moyenne, sont le plus souvent ramenés vers le centre et entourés par le tissu vert. 
L'intéricur de la feuille est occupé par quatre grandes lacunes, les deux supérieures étant les plus grandes, correspondant au milieu des deux moitiés de la feuille; les autres s'alignent à partir de là en diminuant de grandeur. Cette structure moins forte correspond lien aux conditions d'habitat d'une plante qui croit dans les prés humides et lourbeux, dont le sol mou ne présente pas beaucoup de résistance el où l'enchevêtrement des autres végétaux croissant très serrés les uns contre les autr'es, affaiblit ainsi les effets mécaniques du vent.

Entre ces leux formes extrêmes des C. riparia, maxima et provincialis d'une part, où le limbe est aplati et très large, et celle de C. Davalliana, d'autre part, où la section transversale est à peu près triangulaire, nous avons toute une série de formes intermédiaires. De plus, chez plusieur's espèces élrangères à nos contrées, nous voyons le limbe prendre des formes différentes sur les détails desquelles nous n'entrerons pas.

\section{Structure de l'épiderme}

PAPILles - - POILS - Gellules DE PlisSEMENT -

SILIGIFIGATION - APFAREIL STOMATIQUE

Si nous comparons les feuilles de deux espèces, -soit Carex r'emota, d'une part, et Carex Schreberi, de l'autre, - nous royons surgir dans la structure des deux épidermes une différence assez considérable. C'est ainsi que chez la première, nous avons un épiderme très puissamment développé sur la page supérieure el occupant un bon tiers de l'épaisseur totale de la feuille, tandis que dans la seconde, nous trourons, il est rrai, deux épidermes d'inégale grandeur, comme c'est toujours plus ou moins le cas, mais diflérant essentiellement de 
celui de C. remota, par la taille et par la présence de cellules développées en papilles de forme pyramidale et parfois très aiguës.

Chez C. distans, les èpidermes parfaitement lisses sauf toutefois sur les bords de la feuille - présentent, à part la différence de grandeur, la même apparence; tous deux sont formés de cellules régulières arrondies, munies de parois fortes sur le côté extérieur, plus allongées dans le sens de la largeur de la feuille sur la page inférieure, tandis que sur l'autre page, les mêmes éléments présentent un allongement dans le sens inverse, perpendiculaire à la direction du premier.

Si nous comparons maintenant les éléments qui forment la couche épidermique de la page supérieure chez le mêrne G. Schreberi avec les éléments correspondants de la page inférieure chez G. atrata, nous constaterons du premier coup que ces cellules ont pris par leur développement en papilles des formes différentes.

Si chez C. Schreberi nous avons prédominance de la forme pyramidale aiguë, qui est en général celle des papilles développées sur la page supérieure, nous trouverons par contre sur l'autre, chez C. atrata (et aussi chez le même C. Schreberi) la forme bouclée à sommets arrondis, particulière à cel épiderme inférieur.

Remarquons en même temps que chez cette dernière espèce, ce développement est général pour toutes les cellules de l'épiderme, tandis que chez C. Schreberi, nous avons toujours intercalation d'une ou plusieurs cellules non développées en papilles.

Il en est ainsi pour une foule d'espèces, parmi lesquelles je citerai encore C. nigra (fig. 40), C. maxima (fig. 109), C. brizoïdes, C. acuta (fig. 246 ).

Un grand nombre de feuilles présentent un épiderme absolument lisse, aplati, plus ou moins vallonné, l'extré- 
mité des cellules faisant un peu saillie en dehors. A ce groupe à épiderme lisse, appartiennent entre autres les espèces: C. arenaria, C. hordeïstichos, C. leporina, C. alba, C. divulsa, C. muricata, C. vulpina, C. Davalliana, C. dioïca, C. strigosa, ete.

Outre la forme pyramidale que présentent les papilles chez C. Schreberi, nous avons encore d'autres formes, par exemple chez C. provincialis (fig. 169 a. a') où les cellules épidermiques placées vis-à-vis des faisceaux eorrespondant aux nervures principales, prennent des contours tantôt arrundis, tantôt plus ou moins pointus, avec renflement dans la partie centrale de la cellule.

Ces sortes de papilles, en général très nettement différenciées des autres cellules épidermiques par leur grandeur, ne se trouvent que sur la page supérieure de la feuille.

D'autres espèces enfin ne portent ies appendices que sur la page inférieure. (C. stricta).

En coupe longitudinale, les papilles, quel que soit leur contour, présentent le même aspect. Vues de face, il n'ẹ est pas toujours de même, la forme variant souvent de l'ovale à la sphère. (Chez $\mathrm{C}$. provincialis, - nous avons des papilles ovales, rondes chez C. riparia (fig. 146) et chez C. glauca (fig. 96).

Dans les espèces pourvues d'un épiderme lisse ou présentant la forme intermédiaire avec saillies ou vallonnements, les coupes longitudinales nous montrent que ces saillies résultent d'un exhaussement de la paroi, qui a toujour's lieu à la même extrémité de l'élément épidermique (C. Buxbaumii, fig. 65); (C. ornithopoda, fig. 6.)

Ce ne sont pas là les seules formes que l'on rencontre. Il existe dans la plupart des espèces mne autre sorte de prolongements des cellules épidermiques, prolongements en papilles qui ont alors un rôle physiologique plus marqué, 
probablement celui de défendre la plante contre les approches des insectes et d'autres animaux en empêchant la reptation. Ces papilles spécialisées se trouvent sur les arêtes de la feuille, comme aussi sur celles des tiges, dans leurs parties supérieures principalement. Elles offrent la forme générale de crochets plus ou moins épaissis, dont les pointes ont toutes la même orientation et présentent une épaisseur variable (voir fig. 92, 95, 77).

C'est grâce à cette orientation uniforme et à leur disposition en série successive et interrompue que les feuilles et les tiges deviennent scâbres sous le doigt et parfois tranchantes. Chez quelques individus, elles ne se bornent pas à occuper seulement le bord des feuilles, elles se répandent aussi sur la surface de l'épiderme, comme chez C. brizoïdes et C. glauca.

Chez cette dernière espèce, elles présentent une forme de quadrilatère allongé dont un des côtés s'est effilé en pointe (fig. 95).

Quel que søit leur aspect, la genèse des papilles a lieu le plus souvent par développement en hauteur de cellules épidermiques. On observe cependant certains cas où elles semblent résulter du cloisonnement d'une cellule-mère primitive, cloisonnement qui a lieu d'abord par une paroi longitudinale, puis par une seconde et enfin, entre ces nouvelles cloisons, par une troisième, perpendiculaire aux deux premières.

On a ainsi formation d'une paire de cellules jumelles, qui suivant les cas peuvent se développer simultanément. ou dont le développement est limité à une seule d'entre elles.

Cette bipartition en cellules intérieures est facile à constater dans les feuilles jeunes de C. distans et sur la face inférieure de celles-ci (tig. 128.)

Il est cependant difficile d'affirmer que ce soit vraiment 
lả la marche de développement suivie par ces papilles, surtout si l'on a égard à la forme et à la position des cellules-jumelles. Il se pourrait en effet fort bien qu'on ait à faire ici aussi à la naissance d'un stomate dont le développement se trouve arrêté par l'apparition d'une cloison qui, au lieu de se produire longitudinalement, a lieu transversalement.

Nous avons dit au commencement de ce mémoire que la présence de poils nettement développés est un fait rare chez les Carex.

Une seule espèce, parmi celles que nous avons étudiées, C. hirta, est pourvue et, cela très abondamment, de ces appendices, qui sont formés d'une seule cellule à parois minces et terminés en pointe acérée et fortement épaissie. Tout le long de leurs parois se trouvent des aspérités formées à l'extérieur par une ondulation de la membrane cellulaire. Ces poils se trouvent sur la tige et sur les deux faces de la feuille, mais en beaucoup plus grand nombre sur la face supérieure, aussi bien à la base de la plante que dans les parties élevées.

Il n'en est pas de même pour les papilles qui sont localisées quelquefois dans les feuilles occupant une position supérieure sur la tige, tandis que celles voisines du sol en sont gėnéralement plus ou moins dépourvues. Nous en avons un exemple dans deux espèces très voisines, C Schreberi et $\mathrm{C}$. brizoïdes.

Jusqu'ici nous n'avons parlé que des cellules épidermiques modifiées en poils ou en papilles; disons maintenant un mot de celles qui conservent leur forme primitive.

Ce seront les cellules de la page supérieure qui nous intéresseront surtout, celles de l'autre ne présentant jamais beaucoup de variété dans leur forme et dans leur grandeur. 
En effet, tandis que sur la page supérieure, nous pouvons avoir des cellules qui arrivent à doubler et à tripler leur volume suivant leur position et le rôle qu'elles ont à remplir, nous n'avons sur l'autre page qu'une succession de cellules dont l'uniformité n'est rompue que par la présence des stomates ou des papilles.

C'est donc les éléments de l'épiderme supérieur que nous devons considérer plus spécialement.

Leur aspect est très variable : tantòt ils se montrent d'une grandeur uniforme, tan lòt d'un diamètre plus étroit, situés au mème niveau, plus haut on plus bas que les autres éléments épidermiques roisins. Il en est ainsi chez C. strigosa, dont la feuille est depourvue de papilles et dont l'épiderme de la page supérieure présente des cellules beaucoup plus grandes que celle de l'inférieure.

En outre, en regard des principaux faisceaux vasculaires, celui-ci se trouve composé de cellules plus petites et plus enfoncées dans le tissu mème, de sorte que la feuille présente des sillons longitudinaux très marqués.

Les Carex ètant pour la plupart constitués pour une vie plus ou moins aquatique et aérienne, ils doivent présenter un épiderme qui assure à la feuille et aux autres organes une résistance suffisante; c'est ce que l'observation montre parfaitement.

En effet les cas sont rares dans le genre, où la paroi externe des élėments épidermiques ne soit pas fortement épaissie. De plus c'est une loi générale - du moins pour toutes les espèces étudiées ici-que vues de face,ces cellules soient munies de parois ondulées s'engrenant les unes dans les autres, disposition qui n'a d'autre effet que d'assurer à la plante un pouvoir de résistance plus grand contre les pressions extérieures.

C'est ainsi que chez C. provincialis, ces parois fortement zigzaguées apparaissent d'une épaisseur considérable. 
Il en est de même chez C. glauca (fig. 96, 97).

Les fig. 146, $703 b$, et 127 représentent encore cette forme d'épiderme chez les C. riparia, acuta, divulsa et distans.

Comme nous le verrons plus loin, ces mêmes cellules peuvent devenir le siège d'une formation de tannin et d'une silicification particulière. Outr'e celte spécialisation, nous pouvons avoir aussi, soit sur la surface de l'épiderme ou à l'intérieur entre les couches de culicularisation, lesquelles sont souvent très apparentes et forment quelquefois des dessins particulier's au lieu de jointure de deux cellules voisines (C. provincialis, fig. 169 c), des dépôts de corps cireux, réfractant très fortement la lumière, insensibles aux agents colorants, difficilement solubles dans l'alcool, mais disparaissant promptement dans un mélange à chaud de ce liquide avec de l'éther et par un séjour prolongé dans l'eau chaude où ces corps se divisent en gouttelettes qui ne tardent pas à se liquéfier.

Nous en trouvons un exemple intéressant et facile à constater chez une espèce très commune dans nos régions, C. glauca. Si nous laisons une coupe transversale très mince d'une feuille bien développée de ce Carex, nous pourrons d'abor'd constater que les cellules épidermiques présentent dans leurs parois externes un fort épaississement et sont pourvus de lumens de forme régulière, quelque peu quadrangulaire. Au-dessus de chacun des lumens se trouve un corps opaque, en forme de croissant, composé de granulations nombreuses qui, au microscope polarisant, grâce à leur pouvoir biréfringent puissant, apparaissent encore plus visiblement (fig. 98, 105).

Evidemment nous avons affaire ici à une mesure de précaution prise par la Nature, mesure qui a pour but de diminuer la vitalité des organes de végétation au bénéfice d'une maturation plus rapide des fruits ou bien d'obvier 
à une trop rapide évaporation de l'eau dans les tissus sous l'influence des rayons solaires, concurremment avec la couche de cuticule, l'enfoncement des stomates sous l'épiderme et la silicification des membranes. Toutes ces mesures de protection correspondent parfaitement avec les conditions de végétation de cetle espèce qui affectionne les taillis, les bords des haies, les endroits humides, mais où l'eau ne reste pas en permanence. Ce serait donc grâce à ces précautions que la plante peut conserver la turgescence de ses tissus, pendant les époques où le sol qui la nourrit devient moins riche en eau. Peut-être devons-nous aussi voir dans ces phénomènes l'explication du fait que l'eau ne mouille pas les organes verts et ne peut séjourner' longtemps à leur surface.

La même espèce citée plus haut nous offre encore un exemple d'une coloration différente, glaucescente, particulière à l'épiderme de la page inférieure.

Ce phénomène, qu'elle partage avec d'autres, telles que C. riparia,-C. paludosa, C. ampullacca, parait avoir pour cause première le jeu des rayons lumineux entre les nombreuses papilles qui hérissent la page inférieure des feuilles, ou un dépôt de matière cireuse à leur surface.

A l'épiderme de la page supérieure se rattache un tissu particulier, dont la fonction est de servir d'axe à la feuille, lorsqu'elle se replie, mouvement auquel le degré de turgescence de ses tissus l'amène. Ces cellules qui forment en général un groupe bien distinct soit par leur forme, qui varie peu, soit par leur disposition centrale, leur manque de chlorophylle, les parois minces de leurs éléments sur les côtés autres que le côté extérieur, se présentent en une série d'éléments allongés comme les par- 
ties d'un éventail, plus ou moins comprimés et toujours vides de contenu ${ }^{3}$.

Leurs parois ont des contours plissés ou plus ou moins ondulés, quelquefois cependant, on les voit tendues et donner aux cellules un aspect vésiculeux. Ce dernier état est celui que l'on trouve dans les feuilles en pleine turgescence. - La paroi extérieure en est tonjours fortement épaissie, et cet épaississement peut dépasser le double et le triple de celui des parois latérales, lesquelles présentent aussi un pouvoir rérringent considérable et se comportent avec les réactifs comme des membranes formées de cellulose pure.

Traitées par les agents colorants, les coupes de ces cellules ne présentent pas de coloration spéciale, tandis que tout le reste des tissus se teinte d'une façon intense; cependant par un traitement à l'acide sulfurique et à la teinture d'iode, on obtient la réaction bleue caractéristique.

Leur distribution varie suivant les espèces : en effet, chez certains Carex, on les voit former une rangée d'éléments allongés dans le plan perpendiculaire à la surface du limbe, tantôt se différenciant brusquement des autres cellules de l'épiderme, tantôt aug mentant leur lumen insensiblement et graduellement de l'une à l'autre, jusqu'à la grandeur maxima qui correspond alors au faisceau de la nervure moyenne.

A ce groupe se rattachent les espèces suivantes:
C. remota
C. Grayi
C. vulpina
C. alba
C. paniculata
C. praccox (fig. 76)
C. arida
C. muricata
C. arenaria
C. divulsa (fig. 2)

1 Tschirch. Beiträge zu der Anatomie und dem Einrollungsmechanismus einiger Grasblätter. Pringsheim's Jahrb. f. wiss. Bot. Vtll. Duval-Jouve. Histotaxie des feuilles de graminées. Ann. des Sc. Série VI., t. I. 
Au contraire, chez les espères qui suivent, nous avons ces éléments disposés sur deux ou plusieurs rangs formant un tissu d'articulation remarquable par la grandeur de ses cellules, le peu d'épaisseur de leurs parois et leur manque de contenu :
C. hirta
C. paludosa
C. vulgaris
C. sylvatica
C. stricta
C. provincialis
C. glauca (fig. 106)
C. riparia (fig. 148)
C. maxima

Toutes ces cellules d'articulation ne sont pas de même taille, seules celles qui font suite à l'épiderme, - celles du rang le plus extérieur - présentent la forme allongée et agrandie, qu'elles ont habiluellement dans les espèces où elles ne se trouvent que sur un rang. Les autres, - c'est-à-dire les cellules sous-jacentes - forment un parenchyme, dont les éléments de grandeur plus réduite, prennent, par la pression réciproque qu'ils exercent les uns sur les autres, des formes plus ou moins polygonales.

Comme nous l'avons déjà dit plus haut, ces éléments sont tantòt brusquement différenciés des autres éléments voisins de l'èpiderme, tantôt vont-croissant par gradation. Comme exemple du premier cas, citons la feuille de C. præcox (fig. 76), C. nigra, C. brizoïdes, et comme types du second, celles de C. glauca (fig. 106), C. pseudoCyperus (fig. 141), C. vésicaria (fig. 157), C. divulsa (fig. 2), etc. Parfois même, elles sont à peine distinctes de leurs voisines, comme chez C. humilis et C. Davalliana.

Duval-Jouve, en étudiant les mêmes organes, dit que ces cellules lui ont paru « dépourvues de canalicules de communication. $\triangleright$ Cependant il existe des cas, rares il est vrai, où l'on peut constater des penctuations sur les parois latérales de ces éléments. Le C. glauca nous en fournit encore un exemple (fig. 109). Le nombre des 
cellules qui composent ces appareils de plissement varie considérablement. Chez telle espèce, l'on en tromve seulement une rangée de 6 a 7 ; chez telle autre, ce nombre augmente ct atteint le chiffre de 12 à 15 el quelquefois plus; il devient alor's assez difficile de déterminer une limite entre ce qui est cellule épidermique proprement dite et cellule de plissement.

Quant à leur relation les unes avec les autres, on a ici aussi quelques particularités à remarquer: ainsi chez le $C$. hordejstichos, nous pourrons constater, outre une épaisseur considérable des parois, une disposilion collenchymateuse des éléments par rapport à leurs jointures (fig. 175). De mème chez C. riparia (fig. 148), C. nigra, C. glauca (fig. 106), C. hirta, mais à un degré moindre toutefois; il y a en effet des formes de transition jusqu'au cas eù ces jointures conservent leur aspect normal ( $C$. brizodes, C. atrata, C. vesicario, C. divulsa (fig. 2) C. præcox (fig. 76).

Ces épaississements collenchymateux ainsi associés à des parois minces, font que ces cellules présentent à la fois une haute solidité et une grande flexibilité.

En général, la paroi extérieure des cellules de plissement, toujour's fortement épaissie, est recouverte d'une couche de cuticule ondulée, dont les plis, souvent très rapprochés les uns des autres, peuvent faire croire à la présence d'une membrane cornée ou granuleuse; ces plis disparaissent plus ou moins dans l'étal d'extension de la leuille, c'est-à-dire lorsque la plante possède un quantum d'eau considérible, réapparaissent quand l'organe, se repliant par la nervure médiane, montre par là que l'ean ne lui arrive plus en quantité suffisante.

Remarquons encore que celte partie de la feuille est toujours dépourvue de stomates el que chez les espéces où ces organes se trouvent en nombre égal sur les deux 
faces, ils se rencontrent toujours en plus grande quantité dans le voisinage de l'appareil de plissemenı, à sa droite el sa gauche.

Ce n'est pas seulement les cellules de plissement qui changent de forme et qui varient de taille; dans toutes les feuilles, nous avons sur les bords un épiderme composé d'éléments présentant un lumen rétréci en même temps qu'un épaississement des parois extérieures qui s'étend aux parois latérales, de manière à leur donner l'aspect de colonnes renflées et fusiformes (fig. 168).

A la résistance offerte par ces éléments ainsi renforcés vient encore se joindre très souvent celle de petits groupes selérenchymateux sous-épidermiques.

Fréquemment aussi, surtout à l'extrénité des feuilles, la cellule terminale qui forme l'arète se développe en crochet pointu.

Une autre particularilé des cellules de l'épiderme est la faculté qu'elles ont de devenir le siège d'une abondante silicification, laquelle a lieu, pour toutes les espèces, à des degrés d'intensité diffërents. La forme sous laquelle s'opèrent ces dépôts de silice est en général celle de petits cônes, qui peuvent se trouver isolés dans une cellule ou en plus grand nombre suivant les cas. Le plus souvent, on trouve ces corps occupant le centre de la cellule en coupe transversale, tandis que, en coupe longitudinale, ils apparaissent formant une rangée de $3,4,5$ et 6 cônes environ.

Quelquefois on constate qu'il en existe deux séries disposées côte à côte. Suivant l'ancienneté du dépôt siliceux, ils peuvent atteindre ou ne pas atteindre la paroi extérieure de l'épiderme. Ils ralient aussi de taille suivant les espèces; parmi celles qui ont èté étudiées ici, c'esı 


\section{2}

Carex hordeistichos qui en a fourni les plus gros spécimens (fig. 176).

Leur nature siliceuse se révèle par leur résistance aux plus forts acides; si l'on traite en effet une coupe contenant de ces cònes par l'acide sulfurique concentré et l'acide chromique, toute la matière organique est détruite et il ne reste comme résidu que des corps pyramidaux, plus ou moins triangulaires (fig. 100).

En coupe tangentielle, ils se présentent sous la forme de carrés plus ou moins parfaits, au centre desquels s'aperçoit un corps pyramidal (c, c').

Dans la plupart des cas, ils se trourent dans les cellules épidermiques des deux faces et cela, de préférence, dans les éléments plus petits, qui font riś-à-vis au sclérenchyme des faisceaux (fig. 99). Dans d'autres parties du tissu foliaire, je n'en ai pu constaler la présence; d'où nous pourons conclure, que dans le genre Carex et ce doit être aussi le cas pour les autres Cypéracées et les Graminées - la silice se dépose surtout dans l'intérieur des éléments épidermiques et probablement aussi dans leur's membranes.

D’après liohl ${ }^{1}$, ce sont surtout les espèces qui croissent sur les rochers et dans les terrains sablonneux qui se distinguent par la richesse de leur contenu en silice. Ce sont par exemple, les C. arenaria, Schreberi, leporina, rigida, supina, ericelorum, præcox, humilis; toutefois les espèces provenant de prairies humides ou tourbeuses s'en montrent encore très richement pourrues, comme le proure le tableau suivant qui donne en $\%$ le contenu siliceux des cendres de quelques espèces:

${ }^{1}$ Kohl Anat.-physiol. Lntersuchung der Kalksalze et Kieselsaüre in der Pflanze, Marburg 1889. 

C. cæspitosa . 53.25 p. c.
C. remota . . 4 40.1 》
C. vesicaria . . 42.6 »

Le cône siliceux est toujours disposé à l'intérieur de la cellule de façon que la base de la pyramide regarde la couche d'éléments sclérenchymateux el que son sommet plus ou moins aigu corresponde à la partie centrale de la paroi extérieure, ceci s'appliquant, bien entendu, au cas où nous n'avons qu'un seul cône en coupe longitudinale, ou une seule rangée de'ces corps en section transiersale.

Très souvent les cellules silicifères communiquent avec les éléments du sclérenchyme sous-jacent par des ponctuations simples. Chez C. hordeïstichos, ces cônes siliceux, très volumineux, arrivent à toucher de leur pointe la paroi extérieure, qui paraît être aussi le siège d'une silicification abondante, gràce à laquelle son contour paraît plus ou moins accidenté, tantôt pourvu de mamelons arrondis, tantòt émettant des prolongements qui traversent comme des ponts toute la cavité restée libre de l'élément épidermique. Ceci est surtout facile à observer chez les espèces pauvres en cônes siliceux.

La grandeur des cellules silicifères n'est pas constante pour une même espèce; il en est encore ainsi du cône lui-même suivant son âge. En effet, dans beaucoup de cellules, on ne trouve qu'une éminence faisant à peine saillie sur le fond el qui forme le point de départ du còne futur.

Peu à peu, par dépôt de nourelles couches, ce cône, primitivement très arrondi, s'élève jusqu'à occuper presque tout l'espace du lumen. A ce moment la cellule ne paraît renfermer plus autre chose que le corps siliceux, tout autre trace de contenu disparaissant petil à petit.

De quelle utilité peut être à ces végétaux la présence 
de ces corps siliceux? C'esı lả une question qui est assez difficile à résoudre et sur laquelle, on ne peut guère faire que des hypothèses. A-t-on ici aussi une mesure de renforcement des tissus extérieurs dans le but de leur assurer une plus grande solidité? C'est possible, bien que l'on trouve ces corps dans beaucoup d'autres végétaux qui ont d'autres moyens plus puissants de résistance.

Peut-être est-ce une manière de faire de la plante, en vue de se débarrasser d'une façon utile de la trop grande quantité de silice que le sol, sur lequel elle croît, lui envoie par les racines?

A notre avis, il nous semble que nous avons plutôt à faire à une mesure défensive contre les attaques des animaux et spécialement contre la morsure des larves de divers insectes. On comprendra en effet facilement le préjudice que doivent causer aux mandibules de ces dernier's, ces corps pierreux, traitreusement cacliés sous un épiderme déjà très résistant.

Du reste - et ceci pourrait peut-être confirmer notre idée - tous ceux qui se sont occupés d'entomologie savent parfaitement que, si l'on veut récolter des chenilles de lépidoptères, ce n'est pas sur les Carex, ni sur les Cypéracées en général, qu'il faut les aller chercher', car ces plantes possèdent le rare privilège de ne servir de nourriture pour ainsi dire à aucune larve — du moins dans nos contrées.

Il est en outre reconnu depuis longtemps que la silicification du tissu cellulaire constitue un moyen de défense : chacun sait qu'il existe dans l'Afrique tropicale des végétaux herbacés dont le contenu en silice est si considérable, qu'ils deviennent non seulement inattaquables pour les animaux inférieurs, mais encore pour les grands vertèbrés et en particulier pour les bestiaux. Au Japon 
même, pour citer encore un exemple, cette silicification énorme de certaines plantes devient un grand obstacle à l'élevage du bétail.

Dans nos pays, les graminées et autres herbes qui présentent aussi cette silicification hautement développée, mais à un degré bien moindre, peuvent servir souvent de nourriture à nos animaux domestiques, mais il n'en est plus de même pour certains mollusques, tels que nos - limaces, nos escargots, etc ${ }^{1}$.

A l'étude de l'épiderme se rattache celle d' l'appareil stomatique, qui présente, dans le genre Carex, une série de formes intéressantes, souvent caractéristiques pour l'espèce, ce qui dans la détermination peut être parfois d'un grand secour's.

Cependant malgré la diversité que présente l'appareil stomatique, on y reconnait encore partout le mème plan; seulement, par le développement plus ou moins considérable que prennent les cellules annexes, le plan se modifie quelque peu.

Avant de passer à un examen détaillé de ces diver'ses formes, disons un mot de la distribution et de la forme respective de ces organes.

On les rencontre principalement, comme c'est le cas habituel, sur la page inférieure de la feuille, souvent en nombre très considérable et tous orientés dans le sens de la longueur de l'organe qui les porte.

Dans quelques cas, mais plus rarement, ils occupent aussi la page supérieure, en général en nombre plus faible que sur l'autre page et rassemblés surtout autour de

${ }^{1}$ Stahl. Pflanzen und Schnecken, Jena 1888. 
l'appareil de plissement ${ }^{3}$. Cependant il existe des espèces où le nombre des ouvertures stomatiques est aussi grand sur lil face supérieure que sur celle qui lui est opposée. (C. vulgaris, C. Buxbaumii.)

Passant à l'anatomie de l'appareil stomatique, nous le voyons, dans les cas les plus simples, c'est-à-dire dans ceux où l'épiderme est lisse ct où le stomate n'est pas enfoncé dans les tissus, composé de deux cellules semblables laissant entre elles une fente allongée. Ces cellules de fermeture sont en général pourvues de forts épaississements et d'un très petit lumen allongé, dont la forme peut varier aussi. (Comparez par exemple les fig. 21 et 25 b.)

La chambre stomatique est plus ou moins grande, tantôt spacieuse, comme chez C. hirta (fig. 176), C. arenaria (fig. 31) et C. alba (fig. 21); tantôt étroite et peu profonde comme chez C. ampullacea (fig. 35 a). C. vulgaris (fig. 36 b) et C. maxima (fig. $25 b$ ).

Tous ces stomates sont pourvus d'un bec très prononcé qui ferme la fente. Leurs cellules annexes varient aussi de grandeur, comme le montrent les figures $32,24 b$, 33, $29 a, 18$. Vus de face el abstraction faite des prolongements de parois au-dessus de l'ouverture, ils se présentent généralement ( $y$ compris les cellules annexes) sous une forme (lig. 38 ) sphèrique, dont les pôles auraient été considérablement aplatis.

D'autres fois, la forme est parfaitement celle d'un ovale allongé dans le sens de la longueur de la feuille.

${ }^{1}$ Dans cette catigorie se rangent les feuilles des especes suivantes:
C. riparia.
C. pseudo-Cyperus.
C. vesicaria.
C. ampullacea.
C. hordeïsticlıos. 
Dans la majorité de: cas, la vue du lumen de chacune des cellules stomatiques se trouve obstruée par une portion de l'épaississement qui porte les deux becs et qui forme ainsi une bande souvent très épaisse, présentant son maximum de largeur à la hautcur de l'ouverture du stomate (fig. $39 a$ el 39 b). Le lumen est le plus souvent placé obliquement par rapport à la surface de l'épiderme et n'est séparé de la cellule annexe que par une cloison très peu épaisse, tandis qu'à l'opposé, vers la fente stomatique, il se trouve écarté de celte dernière par une paroi très forte. Les figures $39 a, 39 b$, donnent la disposition de ces différentes parties dans leurs relations réciproques.

Tel est le type ordinaire; mais très souvent cet aspect se trouve modifié, quand la feuille acquiert un certain âge el quand l'épiderme est pourvu de papilles. Alors se montrent une quantité de formes plus ou moins élégantes, suivant le degré de développement de ces papilles et des cellules annexes.

Tantôt on aura simplement prolongement de ces dernières comme si elles voulaient se rejoindre au-dessus de l'ouverture, c'est le cas par exemple pour C. vulgaris (fig. $36 \mathrm{~b}$ ), C. acuta (fig. 24 b). C'est aussi ce qu'on trouve, mais déjà différant quelque peu chez C. Buxbaumii (fig. $32 a$ ), C. maxima (fig. $25 b$ ), où les cellules annexes ne font que de s'élever perpendiculairement à la surface de la feuille.

Chez C. stricta, les cellules annexes en forme de haricot proéminent fortement sur les éléments épidermiques voisins qui sont développés en papilles épaissies et viennent entourer le stomate par dessus en formant un puits très profond (fig. 29 a).

D'autres fois, ce sont les éléments entourant l'appareil stomatique qui, se développant fortement, en même 
temps que la paroi supérieure des cellules annexes, viennent en s'avançant toutes deux en sens inver'se, produire un puits trèsprofond, à parois considérablement épaissies, dont le canal s'ouvre au dessus du stomate en forme de cheminée. Une semblable distribution se rencontre chez un grand nombre d'espèces, dont nous citerons seulement les plus importantes:
C. glauca (fig. $33 a$ )
C. paniculata (fig. 306)
C. provincialis (fig. 19. 20)
C. atrata (fig. 41)
C. riparia (fig. $23 a, 23 b$ )
C. ampullacea (fig. 35 a)
C. nigra (fig. 40)

Vus de face, ces stonates ainsi protégés, présentent des dessins variés et souvent élégants. Etudions d'un peu plus près quelques-unes de ces formes curieuses.

1). Carex provincialis. - Chez cette espèce les cellules de fermelure disparaissent complètement sous 3 lobes épaissis et échancrés développés sur les parois des cellules annexes (fig. 19.20).

2). Chez $C$. riparia nous avons formation de 2 corps en forme de $\mathrm{V}$, à branches très fortes et dont les parties ouvertes se font vis-à-ris, cachant à moitié seulement la fente du stomate (fig. 23, $a . b . c$ ).

3). Carex paniculata nous montre des épaississements irrégulièrement distribués; sur les côtés latéraux s'observe en outre une interruption, c'est-à-dire un point où les parois ont conservé leur épaisseur primilive (fig. $30 a b$ ).

4). Chez C. glauca, la forme des dessins se rapproche de celle que nous trourons chez C. provincialis, mais ici les formes restent plus régulières (fig. 34, $33 a$ ).

5). C. ampullacea présente un dessin plus compliqué en ce sens que les extrémités des $V$ paraissent, quelquefois, sans doute suivant la profondeur de la coupe, reliés entre elles par un pont en arc de cercle (fig. 35) et (fig. $35 a$ ). 
Chez les espèces où c'est la cellule annexe qui forme un puits en s'élevant seule au-dessus du niveau de l'épiderme, le stomate présente, vu de face, en regard de son ouverture, un sinus étroit, plus ou moins ouvert suivant les espèces. Comparez entre eux les stomates des espèces suivantes :

C. stricta (fig. 29 b) C. vulgaris (fig. 36 a) C. acuta (fig. $24 a$ ).

A côté de ces formes compliquées, viennent se placer celles qui sont régulières, jusqu'à la fin de leur développement. Ces formes simples sont en majorité.

A ce groupe appartiennent les espèces suivantes :

C. divulsa (fig. 3)

C. Oederi (fig. 18)

C. arida (fig. 28)

C. arenaria (fig. 31)

C. hirta (fig. 17 a. b)

C. alba (fig. 21)

C. distans (fig. 37)

C. depauperata (fig. 26).

C. hordeïstichos (fig. 22)

C. baldensis (fig. 27)

Dans beaucoup de cas, on peut rencontrer, mêlés à d'autres qui sont superficiels, des stomates paraissant enfoncés dans les tissus ou tout au moins dans l'épiderme, grâce à la présence dans leur voisinage immédiat de papilles fortement développées en longueur (C. acuta, C. atrata). Ces papilles, chez C. nigra oü le stomate est entouré de 4 cellules semblables, viennent quelquefois se rabatire sur l'ouverture.

En coupe longitudinale, les cellules de fermeture présentent la même forme que chez les Graminées, c'est-àdire, celle d'un I majuscule, dont les extrémités se seraient arrondies. Suivant les espèces, cet I peut être accompagné d'une ou plusieurs figures en cercles, qui représentent les sections des papilles voisines.

Les stomates ne diffèrent pas seulement par leur forme extérieure, mais encore par leur contenu : dans beaucoup d'entre eux en effet, nous rencontrons des substances autres 
que le noyau, la chlorophylle, l'amidon et le simple protoplasma; beaucoup servent de magasins pour des substances d'assimilation.

Ces matières sont le plus souvent des huiles qui se révèlent dans le champ du microscope par leur grande réfringence, leur quantité considérable, souvent par la taille des gouttelettes et par des réactions dont nous allons dire quelques mots, afin de n'avoir pas besoin d'y revenir.

Le procédé le plus pratique pour déterminer la nature de ces substances a pour base la teinture d'or'canelte. Pour cela, on fait une coupe pas trop mince, que l'on place sur un porte-objet; sur ce porte-objet, et sur la coupe même, on dépose un fragment bien lavé de la racine en question, puis sur le tout, un cover. On ajoutc alors quelques gouttes d'alcool fort et on laisse agir une demi-heure environ.

Au bout de ce temps toute l'huile - à l'exclusion des autres substances, - s'est colorée en rose. Si le séjour sous le cover est trop long, la coloration s'étend aussi aux autres particules qui peuvent se trouver dans les cellules.

Ces huiles, dans tous les essais que nous avons faits, se sont montrées solubles dans un mélange d'alcool et d'éther ou simplement dans l'alcool chaud.

L'acide osmiqne très dilué $(1 \%)$ colore aussi ces goutteleltes en noir ou en brun, mais ce réaclif a l'inconvénient de colorer aussi les tannins et une foule d'autres substances dont la nature est douteuse.

Ces huiles se trouvent soit sous forme d'une très grosse goutte ovale, soit sous celle d'une multitude de gouttelettes répandues sur tout le stomate (fig. 28) et quelquefois dans les cellules avoisinantes, si l'on a pressé quelque peu sur le couvre-objet.

Elles sont surtout très apparentes dans les stomates des espèces suivantes : 

C. arida Muhlbg (fig. 28)
C. humilis
C. vulgaris (fig. 36 a)
C. alba

Chez C. muricata et son proche parent C. divulsa, nous avons bien constaté la présence de corps arrondis très réfringents, colorables aussi par les procédés indiqués ci-dessus, mais les demi-résultats obtenus ne nous permettent pas de certifier chez ces espèces la présence d'huile dans leur appareil stomatique ${ }^{1}$.

\section{Genèse des stomntes}

C'est sur les leuilles de C. distans que ces organes sont le plus faciles à observer dans leur développement.

Si nous faisons des coupes minces langentielles de l'épiderme sur la page inférieure d'une jeune feuille, nous aurons toute une série de stomates dans les différents stades de leur développement. Il est alors facile de voir qu'ils résultent comme chez les Liliaciées, de la division d'une cellule épidermique, qui reste plus petite que les autres et qui est elle-même déjà le résultat d'un cloisonnement transversal dans un des éléments zigzagués de l'épiderme.

Cette division achevée, il y a généralement étranglement entre les deux nouvelles cellules. La plus grande restera élément épidermique, tandis que la seconde donnera naissance au stomate.

Pour cela, il se fera d'abord un cloisonnement longitudinal divisant la cellule initiale en deux cellules de fermeture ; pendant ce temps, celles qui sont situées sur les

1 En suite de nouveaux essais pratiqués sur d'autres échantillons, la présence d'un corps huileux a pu ètre aussi constatée et confirmée chez ces stomales. La présénce et la quantité des gouttes paraissent varier suivant l'époque de l'année. 
côtés se cloisonneront de façon à ne donner qu'un seul segment latéral. On aura ainsi un stomate bordé, en haut et en bas, par deux éléments épidermiques et, sur les flancs, par deux autres qui ne sont primitivement que des segments de ces deux éléments voisins latéraux (fig. 38, 1-6).

Tout le développement se résume donc en un premier cloisonnement transversal, puis en un second longitudinal et en un troisième dans le même sens et double qui a pour théâtre chacun des éléments voisins et pour but la formation des cellules annexes.

Dans les jeunes feuilles, les stomates présentent souvent des différences d'aspect assez marquées. Ainsi chez C. riparia, où ces organes sont dans les vieilles feuilles fortement enfoncés sous l'épiderme épaissi et allongé en protubérances, le stomate devient presque superficiel, les papilles de la surface ne s'étant pas encore complètement développées. On observe en effet que celles-ci, dans les jeunes feuilies, ne forment qu'une simple éminence et ne viennent pas s'étaler au-dessus de l'ouverture stomatique. Ce n'est que beaucoup plus tard, que quittant leur position d'écartement et épaississant leurs parois, les cellules épidermiques forment les dessins compliqués et caractéristiques pour cette espèce.

Chez C. provincialis, nous observons le même phénomène. Ici toutefois, le stomate se trouve constamment enfoncé, et cela profondément, sous l'épiderme. La différence consiste alors en ceci, c'est qu'au lieu d'avoir, comme chez les vieilles feuilles de cette espèce, une sorte de cheminée s'ouvrant par un canal étroit au-dessus du stomate et dont les murs sont formés par les prolongements des cellules épidermiques très épaissies en cet endroit, ces parties de la cellule ont au contraire conservé leur aspect bouclé et une épaisseur de paroi uniforme et peu considérable. 
Chez C. glauca qui possèdle des stomates analoğues, nous avons aussi observé le même processus. - Il va de soi que les parois restant minces, on obtiendra un lumen plus grand, et plus arrondi (fig. 102; el 33 c.b. a).

Dans le tout premier stade, les éléments qui doivent former le stomate, apparaissent à niveau des autres éléments de l'épiderme; c'est à peine, si, par endroit, l'on aperçoit, sur le pourtour de la feuille, une dépression au fond de laquelle se préparent les cellules stomatiques, cellules dont le développement marchera le plus souvent de pair avec celui (sur la page supérieure surtoul) de papilles longues et très aiguës (lig. 103).

\section{Développenent de In feuille}

Avant d'entreprendre l'analyse des tissus assimilateur's et conducteur's de la feuille, disons un mot de celle-ci dans son premier âge.

La toute jeune feuille, quelque temps après sa sorlie de la graine, présente en général une forme un peu différente de celle qu'elle acquerra plus tard à l'état adulte : elle est plıs arrondie, pourvue d'un épiderme déjà bien constilué, qui, composé sur la page supérieure d'élćments grands, incolores, à parois minces à l'intérieur, plus fortes à l'extérieur, et se distinguant très lacilement du parenchyme vert par leur forme.

Les autres cellules épidermiques, qu'on les prenne sur l'une ou l'autre des faces de la feuille, présentent la môme forme régulière, mais déjà plus allongée dans le sens tangentiel sur la page inférieure.

A cet àge, en fait de cellules mécaniques, il n'y a que deux ou trois endroits où l'on puisse constater la présence 
de petits groupes d'éléments fibreux ; c'est de chaque côté des cellules de plissement, à gauche et à droite sous l'épiderme (fig. 103) $(\mathrm{m})$.

L'espace qui plus tard sera occupé par les cellules de plissement, l'est en ce moment par un groupe d'éléments, très gros, à parois minces, à grande cavité, de forme polygonale et disposés sur deux rangs, ceux du rang extérieur égalant en grandeur ceux de l'intérieur et repoussant ces derniers dans les tissus dans la direction de la nervure médiane $(e, e)$.

Quant aux faisceaux libéru-ligneux, on commence à en distinguer trois, l'un très gros, correspondant à la nervure principale; les deux autres, au milieu des deux moitiés du limbe, entourés encore de cellules incolores rondes formant une gaine parenchymateuse (cellules aqueuses).

Entre ces trois faisceaux, on peut, suivant les coupes, constater encore une différenciation en vaisseaux chez quelques éléments situés au centre du mésophylle. Tout le reste de l'espace compris entre les épidermes est rempli par des cellules rondes, riches en chlorophylle.

Outre les éléments fibreux qui se rencontrent sous l'épiderme, de chaque côté des cellules de plissement, on voil apparaître aux extrémités du faisceau médian un commencement de gaine fibreuse, représentée par quelques cellules déjà lignifiées, à parois épaisses et à lumens étroits.

Ces cellules vont se substituer peu à peu à la gaine parenchymateuse, qui se trouve être de plus en plus refoulée sur les côtés. A leur tour, ces cellules aqueuses prenant du développement, augmentent de diamètre, tout en gardant des parois très minces, de sorte qu'au bout de peu de temps, grâce à leur contenu incolore, à leur volume plus grand et à leur forme ronde, elles tranchent vivement sur l'ensemble du tissu assimilateur vert. 
De leur côté, les éléments de la gaine fasciculaire ${ }^{1}$ se différencient: de minces (ju'ils étaient d'abord, comme les autres cellules voisines, ils s'épaississent de plus en plus, sur leur côté interne surtout.

Lorsque les cellules épidermiques de la page supérieure ont pris un développernent plus considérable, on les voit dépasser en grandeur celles de la page inférieure, les éléments constituants de l'appareil de plissement, surtout ceux du premier rang s'allongent, puis augmentant de volume, se compriment mutuellement les uns les autres, de façon à prendre plus ou moins la forme d'un éventail à demi-ouvert.

A ce moment seulement se montrent les cônes siliceux ; à mesure que les revêtements fibreux se développent, ils apparaissent en plus grand nombre. Il en est aussi de même pour les cellules sécrélrices dans le tissu assimilateur; toutefois, chez certains Carex (C. glauca), ces cellules apparaissent dès l'origine et en très grande quantité.

Dans les feuilles très jeunes, la partie centrale des tissus ne renferme point encore de lacunes. Celles-ci n'apparaissent que plus tard et semblent résulter de la résor'ption d'un groupe de quatre ou cinq cellules situées entre les faisceaux.

On observe, en effet à cet endroil du mésophylle, au commencement de la formation de ces espaces, que plusieurs éléments perdent leurs grains de chlorophylle et

'Afin d'éviter toute confusion, nous emploierons pour désigner la rangée de cellules à parois épaisses et sıuvent poncluées, qui enveloppent les deux parties du faisceau, le terme de gaine fisciculaire ou simple_ ment de gaine, réservant celui de gaine fibreuse ou de revètement, pour l'enveloppe de cellules sclérenchymaleuses, généralement formée de plusieurs couches, qui protège lout le faisceau y compris sa gaine fasciculaire. 
s'agrandissent, surtout ceux qui occupent le centre du groupe.

On a ainsi formation d'une rosette de cellules incolores, dont les parois par leur résorption successive, laquelle se continuera plus tard, formeront ces lacunes aérifères, qui sont constantes pour toutes les espèces. Il est possible encore qu'elles résultent des rosettes centrales par formation entre les parois des cellules de méats, qui allant croissant toujours, finiraient par former ainsi un espace à l'intérieur de la feuille. Mais l'examen des diver'ses coupes que nous avons faites, semblent plutôt indiquer une lésorption successive des cloisons.

Telle est la façon dont se comportent les différentes parties de la feuille dans son jeune âge. Voyons maintenant ce qu'elles deviennent, lor'sque celle-ci est arrivée à son complet développement. L'épiderme a déjà été truité, il nous reste donc à parler du tissu assimilateur, des fais-. ceaux et de leurs parties constitutives.

\section{Tissu assimilateur}

Les intervalles entre les nervures du limbe et les deux épidermes sont remplis par les lacunes et par une couche plus ou moins épaisse de parenchyme riche en chlorophylle.

Ce parenchyme se présente sous les trois aspects habituels, à savoir sous celui de tissu spongieux, de tissu palissadé et de tissu à cellules plus ou moins hexagonales pouvant laisser ou ne pas laisser entre elles de méats intercellulaires. Comme chez beaucoup d'autres monocotylédones, ces cellules son t disposées à partir de l'épiderme en séries radiales et tangentielles, laissant entre elles des méats, surtout dans la région qui avoisine l'épiderme de 
la page inférieure. Leur forme est sur l'autre face de la feuille généralement plus allongée dans le sens du plan perpendiculaire à la surface du limbe, de manière à présenter un aspect un peu palissadé. Beaucoup de ces éléments offrent, surtout en coupe longitudinale, de petits cercles intérieurs, qui résultent de la section transversale d'unc cellule en palissade, vue par dessus.

Rarement le tissu parenchymateux parait vert sur toute son étendue ; le plus souvent en s'approchant de la région des lacunes, les cellules deviennent plus grandes et forment un tissu en mailles làches qui ne contiennent plus que fort peu de chlorophylle. Par contre, les méats cellulaires s'y trouvent développés sur une grande échelle, si bien que toutes les parois des éléments qui constituentces mailles, prennent des contours ondulés et s'ernboîtent les uncs dans les autres. Telle est par exemple la structure du mésophylle chez la plupart des espèces, et notamment chez C. pseudocyperus, G. distans el C. hordeistichos fig. 179) (fig. 142).

Chez la première de ces espèces, ces cellules à méats forment une série continue oủ toutes les membranes présentent un ou plusieurs de ces méats, le plus souvent un grand suivi de deux plus petits (fig. 142).

Ce ne sont cependant pas lá les seuls endroits où l'on rencontre ces espaces - ou pour être plus précis ces hifurcations partielles des membranes de deux cellules contiguës - on les retrouve aussi, mais isolées el disséminées, dans le tissu palissadé, principalement dans les régions qui avoisinent l'épiderme des faces inférieure et supérieure (fig. 119 et 145), oủ leur grandeur peut devenir très considérable.

En général leur orientation est la même, c'est-à-dire qu'elles se rencontrent le plus souvent, lorsqu'on considère une coupe transversale, sur les parois radiales des cellules. 
Dans beaucoup de cas aussi, leur distribution parait ne pas présenter d'uniformité; on les trouve en effet sur toutes les parois indifféremment, qu'elles soient radiales ou tangentielles.

La présence de ces bifurcations, dont on retrouve en partie l'analogue dans la racine et dans le rhizome, nous paraît avoir plusieurs fins:

10 Une action mécanique qui a pour but de fournir au limbe de la feuille une résistance plus grande par le fail de ses courbures dans le milieu des membranes. Ce serait là l'unique but de leur présence dans ces grandes cellules centrales et incolores.

$2^{0}$ Une fonction physiologique : augmenter dans les cellules à chlorophylle la surface interne de la membrane cellulaire et y engendrer plus d'espace pour la multiplication des grains de chlorophylle.

$3^{\circ}$ Une autre fonction physiologique touchant la circulation et rendue plus probable et plus vraisemblable par le fail que les bifurcations sont beaucoup plus visibles en coupe longiludinale qu'autrement; à savoir que, par suite de leur orientation uniforme, elles contribuent au principe du transport le plus rapide possible des produits d'assimilation, en les empêchant de se disperser dans la masse des tissus en tous sens.

$4^{0}$ Une troisième fonclion, physiologique aussi : l'aération des tissus, au même titre que les lacunes dans le mésophylle.

Sur une coupe tangentielle, le tissu sous-épidermique, plus ou moins palissadé, apparaît le plus souvent pourvu de nombreux méats intercollulaires qui donnent à toute cette partie de la feuille un aspect spongieux; c'est du reste de tout l'organe celte partie qui est la plus riche en chlorophylle, cette dernière diminuant à mesure yu'on s'approche du centre. Sur les coupes transversales, ces 
méats deviennent invisibles pour la plupart et disparaissent quelquefois compiètement.

Nous avons vu plus haut quelle était l'origine des grandes lacunes qui occupent le centre des tissus. Dans les feuilles parvenues à leur complet développement, elles prennent une forme plus ou moins arrondie on carrée et ont leur grand diamètre orienté de deux façons; tantôt dans le sens du plan de la feuille (C. vesicaria) le cas en général pour celles qui sont situées sur les côtes de la nervure médiane - tantôt perpendiculairement à la surface de la feuille (G. humilis), souvent aussi on trouve les deux cas réunis dans. le même organe (C. riparia).

Chez certains Carex, comme C. pseudo-cyperus et C. vesicaria, les lacunes prennent un tel développement qu'elles digèrent presque toutes les cellules assimilatrices, ne laissant de celles-ci que deux ou trois couches au plus autour des faisceaux et sous l'épiderme.

Chez d'autres, au contraire, elles restent assez petites (C. provincialis), s'arrondissent ou prennent des formes irrégulières (C. Davalliana).

Faisceaux libéro-ligneux. - Ils présentent le type des faisceaux normaux des Monocotylédones. On y remarque d'abord une gaine qui les environne complètement ; cette gaîne est, suivan les cas, plus ou moins distincte des cellules fibreuses qui l'entourent et présente des épaisseurs variables dans les parois de ses éléments. En effet, tantôt l'anneau est composé de cellules présentant une même force sur tuut le pourtour, tantôt les épaississements sont localisés dans les éléments qui entourent le liber ou répartis dans les deux ou trois cellules qui, de chaque côté, font vis-à-vis à la ligne de séparation du bois et du phloëme. Ces épaississements ne regardent toujours que le côté interne de la cellule, qui 
acquiert alors un nombre plus ou moins grand de ponctuations.

Les éléments de celte gaine fasciculaire peuvent acquérir une épaisseur remarquable, comme cela a lieu chez certaines espèces qui habitent les marais ou les terrains rocheux de la montagne (fig. 177).

$\Lambda$ l'intéricur se l'ouvent en général les deux parties du faisceau bien distinctes. L'une, la partie libérienne, est formée de cellules à lumens larges et d'autres plus petites à lumens plus étroits, disposées suivant un ordre assez rógulier (cellules annexes). Tout ce tissu prend sous l'action dur chlorme de zinc iodé, une couleur blene qui dénote la non-lignification de ses membranes; très souvent aussi, à l'extrémité dı phloëme ci-dessus décrit, on apcrçoit un groupe d'éléments à membranes gonflées et à lumens très étroits, qui forment une protophloëme neltement circonscrit.

L'autre partie, constituée par les éléments du bois, porte sur les côtés et au milieu du faisceau total deux lumens larges, qui sont les sections de deux gros vaisseaux réticulés, reliés entre eux transversalement par une bande de vaisseaux plus petits. A la suite de ceux-ci s'élagent, tantôt deux par deuxx, tantôt plus irrégulièrement, d'autres vaisseaux (annelés, scalariformes, réticulés, etc.,) accolés pour la plupart les uns aux autres sans interposition de parenchyme ligneux, à parois épaisses, de façon à donner à la partie bois une forme plus on moins allongée.

Les deux partics, liber et bois, sont enclicissées dans un revêtement de cellules sclérenchymateuses, se colorant en brun rouge par le chlorure de zinc iodé, en rouge vif par la phloroglucine et l'acide chlorhydrique ou encore par la fuchisine ammoniacale.

Ce revêtement est formé de cellules plus grosses, à 


\section{1}

lumens plus grands aux abords du faisceau, mais qui, peu à peu, à mesure qu'elles approchent de l'épiderme, deviennent ordinairement plus petites et plus résistantes dans leurs parois. Sur les flancs du faisceau le revêtement se trouve presque toujours interrompu de façon a permettre au parenchyme de s'approcher de la gaine; on remarque alor's que c'est précisément là, que pour les éléments de cette dernière, a lieu l'épaississement maximum des parois internes.

Très souvent sur les coupes transversales, il arrive que deux faisceaux voisins s'anastomosent entre eux en s'incurvant et en envoyant à travers les lacunes une ramification. Ces conjugaisons interfasciculaires sont drjjà visibles à l'œil nu et deviennent très nettes, lorsqu'on traite la feuille par la fuchsine ammoniacale qui colore les fibres d'un beall rouge à l'exclusion de tout autre tissu.

Les faisceaux ront en diminuant de taille a parlir de la nervure médiane et des deux nervures principales. Les plus développés sont donc ceux qui correspondent aux grandes nervures et en particulier à la nervure médiane.

Là, en effel, on ohserve que, concurremment avec les cellules de l'épiderme déreloppées en cellules de plissement, ces faisceaux munis de leurs revêtements sclérenchymateux, occupent toute la largeur de la feuille; rarement entre les deux parties s'interposent quelques éléments du tissu chlorophyllien. Pour les autres faisceaux, on observe quelques différences dans la distribution des éléments mécaniques. Ceci nous amène donc à parler du stéréôme dans la feuille des Carex.

\section{Appareil de soutien de la feuille}

Les Carex élant, pour la plupart, des plantes habitant. les marais, les cours d'eau el aussi, dans beaucoup de 
cas, des terrains plus ou moins durs, arides et dénudés, ont besoin, pour pouvoir résister aux efforts des rents sur leurs feuilles minces et allongées, ainsi que sur leurs tiges élevées, et pour pouvoir acquérir des points d'appui suffisamment résistants, d'une distribution spéciale dans lous leurs tissus, d'élérnents chargés de pourvoir à celte fonction.

Nous avons déjà constaté, dans les lignes qui précèdent, plusieurs autres dispositions analogues, concourant au même but : c'étaient d'abord les zigzags des cellules épidermiques, s'emboitant les unes dans les autres, c'était aussi l'anastomose de quelques faisceaux pendant leur course, la cuticularisation puissante des membranes extérieures de l'épiderme, leur silicification et la présence à l'intérieur des tissus de membranes bifurquées ayant pour fonction, celle d'empêcher le ployement trop brusque et par conséquent la rupture du limbe.

A toutes ces mesures prises par la nature contre les déchirements et contre des chocs venant de l'extérieur, vient s'ajouter l'effet des cellules du stéréôme, cellulesqui présentent, comme on le verra, une distribution différente suivant les espèces.

D’abord nous pouvons constater une chose, c'est que tout faisceau, pourvu qu'il soit développé quelque peu, présente à ses deux extrémités un groupe de cellules fibreuses, qui en coupe transversale, ont une forme polygonale et un lumen petit, et en coupe longitudinale par contre sont très allongées, fusiformes pourvues de parois, fortement lignifiées et richement ponctuées.

Chez les jeunes faisceaux qui sont d'abord entourés par une gaine de cellules parenchynateuses incolores, on voit bientôt celle-ci faire place à une ou deux cellules à parois épaisses, de forme différente, qui apparaissent successivement aux deux extrémités du faisceau. 
On a donc ainsi deux points de départ à partir desquels ces cellules se développent de plus en plus et finissent par former deux petils groupes en forme de croissants qui vont à la rencontre l'un de l'autre. Peu à peu le groupe inférieur multipliant ses éléments, arrive à toucher l'épiderme, dont il comprime les cellules qui deviennent plus étroites et se pourvoient de cônes siliceux. A l'autre extrémité du faisceau, les choses ne se passent plus de même. En effet, ou bien le revêtement formé, s'étendant le long du bois, garde sa forme primitive de croissant, ou bien il y a en outre prolongement du stéréôme vers l'épiderme supérieur, sous forme de colonnes plus ou moins étranglées, formées suivant les endroits par une série d'éléments allant deux par deux et accompagnés durant tout leur trajet par les éléments incolores de la gaine parenchymateuse.

Quelquefois ces colonnes sont formées d'un plus grand nombre de cellules et présentent des rétrécissements où viennent se loger les cellules du parenchyme ambiant. C'est le cas par exemple pour C. provincialis (fig. 169 a.s.) où les colonnes se présentent tantôt formées par deux cellules juxtaposées, tantôt par quatre ou cinq et même plus. En règle générale, elles finissent toutes par s'étaler sous l'épiderme supérieur où elles provoquent un aplatissement des éléments et l'apparition des cônes siliceux (fig. 99 el 105).

Les deux croissants ainsi formés viennent à la rencontre l'un de l'autre sur les côtés du faisceau; ils ne sont souvent séparés que par une cellule du parenchyme; d'autres fois, ils restent à une assez grande distance l'un de l'autre; c'est le cas pour les espèces qui possèdent des faisceaux très allongès (C. humilis).

Chez C. Hordeïstichos, ces revêtements très puissamment développés aux deux bouts du faisceau, traversent 
tout le parenchyme formant une colonne de soutien très épaisse, qui s'étale largement sous les deux épidermes (fig. 178).

Dans la feuille de G. pseudo-Gyperus, nous avons la même disposition, mais ici la partie du stéréôme qui touche à l'épiderme inférieur est plus développée que l'autre, cette dernière s'étant allongée en colonne mince pour venir se terminer sous l'épiderme supérieur en forme de massue (fig. 140).

Il existe aussi des espèces chez lesquelles les colonnes, si elles se forment, we le font que très tardivement ( $C$. Davalliana nous en offre un exemple). Il se forme à leur place, sous l'épiderme supérieur en regard des faisceaux, un petit groupe de cellules fibreuses qui tendent à se propager à la rencontre du revètement du faisceau, de sorte que dans les feuilles àgées, la jonction arrive à se faire plus ou moins complètement. De semblables groupes se forment anssi aux bords des feuilles, mais restent isolés sous l'épiderme, c'est-à-dire n'ont pas de relations avec les faisceaux qui sont dans cette région très petits et entourés complètement de leurs gaines de cellules aqueuses. Deux groupes fibreux semblables sont représentés dans la fig. 1 (en $\mathrm{m}$ et $\left.\mathrm{m}^{\prime}\right)$.

Dans les vieilles feuilles ou les lacunes ont acquis une grandeur très considérable, c'est contre ces colonnes, en même temps que sous l'épiderme, que viennent se réligier les cellules assimilantes.

Nous aurons du reste encore l'occasion de revenir au stéréôme en parlant de la tige.

\section{Tissus parenchymateux}

\section{CELlules aQueUses - CELlules TANNIFìres}

A l'étude des faisceaux et de leurs colonnes se rattache aussi celle de ccs cellules grandes, incolores, qui les 


\section{5}

accompagnent constamment, en formant autour d'eux des gaines qui, lorsqu'ils sont jeunes, les entourent complètement.

Ces cellules nous ont paru ne pas renfermer autre chose qu'un protoplasma aqueux, incolore, sans aucune trace de chlorophylle el ne donnent pas de réaction avec les réactifs ordinaires. Leurs fonctions consistent probablement à servir de pont entre le tissu assimilateur et le tissu conducteur, en donnant passage aux matières assimilées. Elles ont toujours des parois minces et présentent en coupe longitudinale une forme tabulaire allongée.

Dans la plupart des cas, elles se trouvent, dans une feuille bien développée, représentées par quelques éléments disposés en série sur les côtés des faisceaux, série qui se continue le long des éléments mécaniques, mais le plus souvent avec inter'ruptions.

Dans d'autres cas, on les voit former au-dessus des faisceaux et entre les groupes sclérenchymateux situés sous l'épiderne, des colonnes plus ou moins épaisses, lesquelles se continuent quelquefois des deux côtés sous l'épiderme, se rejoignent avec les mêmes cellules du faisceau voisin de façon à enclore presque complètement le tissu assimilateur. C'est le cas qui se présente chez C. provincialis (fig. 170 et169 a). Nais le plus souvent on n'observe pas de jonction sous l'épiderme, ces cellules se contentant de former un tissu incolore à l'extrémité supérieure du faisceau et sur ses còtés. C'est dans ce tissu qu'apparaissent plus tard les cellules lignifiées qui devront constituer la colonne de fibres (fig. 1, C. divulsa).

\section{Cellules tannifères}

Les cellules parenchymateuses disposées en gaîne autour des faisceaux ne sont pas les seuls éléments qui 
se fassent remarquer au milieu du tissu vert, par leur aspect incolore. Il en existe encorc d'autres qui ont la mème forme en coupe transversale et dont la nature ne nous a pas été révélée dès l'abord.

Elles sont tantôt placécs au hasard, disséminées dans le tissu vert au voisinage des lacunes, sous l'épiderme et mème aussi au milieu des débris de cellules qui remplissent les lacunes. Leur indifférence aux réactifs nous les a d'abord fait regarder comme étant les équivalents des éléments décrits dans le paragraphe précédent, mais d'une part, leur présence en grand nombre, leur position souvent régulière à gauche et à droite des revêtements mécaniques, et d'autre part, en coupe longitudi. nale, leur aspect allongé en forme de sac à contours plus ou moins arrondis, nous ont bien vite persuadé qu'il s'agissait d'un autre genre de cellules.

En faisant agir l'acide osmique, le contenu de ces cellules incolores devient d'un beau noir, mais en faisant la réaction avec la teinture d'orcanette, on n'obtient aucune coloration rouge. Ce ne pouvait donc être une matière huileuse emmagasinée. Nous eùmes alors l'idée d'appliquer les réactifs du tannin et ce fut alors avec un plein succès.

Les réactions employées pour nous convaincre de la présence du tannin ont été les suivantes:

10 Perchlorure de fer. Les coupes traitées par cette substance ont donné la réaction caractéristique du tannin, c'est-à-dire une coloration blene, plus ou moins verdâtre ou noirâtre.

Le procédé au fer n'est pas très rapide; il est préférable, pour le bon succès de la recherche, de laisser séjourner une feuille pendant 24 heures dans la solution. On est àlors sùr d'obtenir une coloration générale, mieux qu'avec une simple coupe; il arrive en effet très souvent 
que le rasoir en sectionnant ces cellules, les font se vider en partie ou complètement, ce qui fait que l'on n'obtient plus de coloration.

$2^{\circ}$ Bichromate de Potassium. La réaction est ici instantanée et donne une coloration jaune-brune, beaucoup plus forte et plus générale, lorsque les coupes on séjourné quelques heures dans le liquide.

$3^{0}$ Molybdate d'Ammonium et chlorure de même base. Cette réaction indiquée par Gardiner ${ }^{1}$ et recommandée par Strassburger est en tous points excellente. On fait dissoudre dans une dissolution concentrée de chlorure d'Ammonium un excès de molybdate de même base. Puis dans la dissolution obtenue, on laisse tremper les coupes préparées.

Instantanément aussi, ces cellules incolores prennent une couleur rouge-feu caracléristique, en même temps qu'il y a formation d'un fin précipité granuleux.

Celte dernière réaction et la précédente constituent deux réactions propres au tannin, et ne laissent aucun doute sur la présence de ce corps dans ceriaines feuilles du genre Garex.

Les Cypéracées peuvent donc, elles aussi, être pourvues de cellules sécrétrices, malgré l'opinion jusqu'à présent reçue ${ }^{2}$ que certaines familles, entre autres celle qui nous occupe, étaient complètement dépourvues d'organes sécréteurs.

Les espèces de Carex chez lesquelles se rencontrent ces cellules tannifères sont assez nombreuses. Voici, à titre de renseignement les principales d'entre elles ${ }^{3}$; les

1 Gardiner. Proceedings of the Cambridge Phil. Soc. Vol. IV.

2 Julius Sachs dans ses « Vorlesungen über' Pllanzenphysiologie»1887. dit page 186: «Relativement rares sont parmi les Phianérogames, les familles qui ne possédent aucun organe (réservoir) à sécrétion [Secretbehälter], comme les Graminées. les Ciypéracées et les Renonculacées. »

${ }^{3}$ Pour les espèces qui en sont dépourvues, voir la partie spéciale. 
premic̀res indiquées sont celles où on les rencontre en plus grand nombre) :
C. glauca
C. stricta
C. Buxbaumii
C. distans
C. humilis
C. nitida
C. punctata
C. provincialis
C. alpestris
C. strigosa
C. maxima
C. riparia

La plupart du temps, elles se trouvent comme il l'est dit plus haut, disséminées dans le tissu, en plus grand nombre sur le pourtour des lacunes, mais on peut aussi les rencontrer dans l'épiderme tnême el à l'intérieur des faisceaux.

Leur forme est généralement ronde, plus allongée en coupe longitudinale; quelquefois pourtant elles prennent aussi la même forme en coupe transversale (voir fig. 120, 130 chez C. distans; - fig. 115 C. strigosa; fig. 131 chez C. punctata; - fig, 105 chez C. glauca, où elles présentent la disposition régulièr'e à droite et à gauche de chaque colonne de sclérenchyme.)

Chez les espèces douèes de cellules tannifères (comme C. glauca,) l'étude des jeunes feuilles nous montre qu'elles apparaissent dès le premier âge et même en grand nombre.

Quant au rôle que doivent jouer ces réservoirs à tannin, il n'est guère facile de se l'expliquer d'une façon certaine.

On sait cependant que dans les plantes le tannin joue divers rôles. D'abord il peut n'être qu'une forme chimique, sous laquelle la plante se débarrasse des substances qui lui sont inutiles ou nuisibles en les confinant dans ces cellules qui ont terminé leur existence et qui deviennent ainsi un lieu de dépôt pour ces substances désormais sans emploi.

D'autre part, il est bien certain qu'il possède des propriétés tout à fait spéciales à lui, propriétés qui consistent premièrement à être une des formes transitoires de 
produits destinés à l'assimilation, secondement à rendre imputrescibles les tissus qu'il imprègne, à protéger la surface des plantes, à rendre l'écorce, le bois de cneur des arbres plus résistant et troisièmement peut-ètre, par son hygroscopicité, à ètre un obstacle au dessèchement de ces tissus et un moyen de reconstituer en eux la turgescence qu'ils ont plus ou moins perdue.

On peut sans doute se représenter ainsi le mode d'action, l'utilité de pareilles quantités de cette substance, qui, la plupart du temps, - demeure dans certains tissus, sans subir de transformations ultérieures.

Mais, à notre avis, il nous semble que nous avons plutôt affaire ici aussi à une mesure de défense contre l'attaque des animaux inférieurs. L'action du tannin leur est en effet nuisible, un grand nombre d'expériences ont été faites à ce sujet, el toutes tendent à prouver que le rôle des tannins dans certaines plantes est l'équiralent de celui de la silice déposée dans les cellules de l'épiderme. Peut-être aussi, est-ce tous ces rôles réunis qu'il faut attribuer aux cellules tannifères?

Avant de terminer avec la feuille, récapitulons un peu les moyens de défense que la nature a mis au service de ces plantes en apparence si modestes.

Ils peuvent ètre de deux sortes:

a) ou chimiques,

b) ou résulter d'une disposition particulière, d'une adaptation d'un organe en vue du but proposé.

C'est ainsi que nous avons:

1) Une forte silicification des cellules épidermiques, silicification qui se traduit par la formation de cones siliceux destinés à former un squelette de résistance à la plante et à la protéger contre les attaques des insectes et des animaux plus gros.

2) Du tannin, répandu à profusion dans les tissus, 
tannin qui peut être ou bien une forme chimique provisoire des substances d'assimilation, ou bien une autre lorme chimique dans laquelle viennent se convertir les substances inutiles à la plante, ou bien encore une arthe défensive contre les anirraux comme plus haut.

3) Une forte culicularisation yui aura pour but d'empècher une trop rapide évaporation de l'eau dins les lissus. Ceci concurremment avec les stomales enfoncés sous l'épiderme.

4.) Des dépôts de matière cireuse sur la surface et dans les couches d'épaississement de la paroi extérieure de l'épiderme, dans le inême but que précédlemment (en 3) et peut-être aussi comme moyen de délense analogue au tannin et la silice.

5) Des crochets acérés, orientés tous d'une facon semblable, rabattus sur l'organe qui les porte, de manière à empêcher la reptation ou la morsure de certains animaux.

6) Des matières huileuses enfermées dans les stomates et qui sont une forme chimique sous laquelle des substances nécessaires à la plante se trouvent mises en réserve.

7) Des bifurcations ou plissements de membranes, des lacunes dans les tissus, un système cormplet de cellules formant pour ainsi dire la charpente de l'organe, qui assurent à lit plante une plus grande résistance aux attaques des vents el autres pressions extérieures.

Nous voyons donc que sous tous les rapports, nos Carex sont des mieux pourvus pour la lutte de l'existence. Ce qui d'ailleurs est dit ici pour la feuille se répète aussi plus ou moins pour la tige, le rhizome et la racine, à l'étude desquels nous allons procéder. 


\section{1}

\section{ETUDE DE LA TIGE}

Cette étude peut se diviser en deux grandes parties: dans la première nous trailerons des liges rertes aériennes, dans la seconde nous analyserons les différentes parties qui composent liz tige souterraine, autrement dit le rhizome.

\section{A) De Ia tige aérienne en général}

Nous avons ici, comme pour la feuille, un plan général, une uniformité de dispositions qui se retroure partout, peut-être encore d'une façon plus marquée que pour l'or'gane précédent. En effel, nous ne trourons pas ici de différenciation dans les cellules épidermiques en cellules de plissement, nous n'y trourons pas non plus deux sortes d'épidermes, différant de forme, parce que l'un étant supérieur, l'autre est inférieur; à peine peuton même observer quelques divergences dans le nombre des faisceaux, leur arrangement, etc.

Cette étude d'ailleurs présentera de nombreux points d'analogie avec celle de la feuille. Nous verrons apparaitre ici une foule de caraclères communs: c'est ainsi que nous aurons aussi des cellules i cônes siliceux, des stomates identiques, des bifurcations dans les membranes, des cellules tannifères, des poils, des papilles, et ainsi de suite.

La tige, qui a pour origine un point régétatif arrondi, recouvert par les jeunes feuilles qui forment au-dessus de lui une sorte de casque protecteur, présente, lorsqu'elle a terminé sa croissance, la forme triangulaire d'une façon plus ou moins tranchée. C'est le cas par exemple pour 
les C. riparia, G. vésicaria, C. vulpina, C. atrata el pour beaucoup d'autres. Nais cette forme, pour une même tige, varie quelque pen suivant l'endroit considéré : telle espèce présentera à la base des angles beaucoup plus arrondis, jusqu'à prendre souvent une forme à peu près ronde, puis a mesure que l'on remonte cette tige, les angles s'aecentuent et la forme devient dans le haut nettement triangulaire; telle autre, an contraire, commencant dans le bas par les mèmes contours arrondis conservera plus ou moins ces formes jusque dans le haut de la tige, près de son somment. D'autres enfin resteront de haut en bas nettement triangulaires arec des angles très aigus. A la première catégoric se rattachent les espèces suivantes:
C. chorhiza
G. Baldensis
C. Oederi
G. hirta.

A la seconde :

C. Davalliana,

et à la troisième, les espèces citées plus haut, à savoir :
C. resicaria,
C. atrata.
C. riparia,

En général, toutes ces tiges portent dans le haut surtoul, sur leurs arêtes les mêmes poils-crochets, dits "physiologiques" que l'on a étudiés sur les bords des feuilles; leur fonction y est évidemment la même; ce sont eux qui donnent au doigt celte sensation rugueuse bien caractéristique, lorsqu'on le promène sur les arêtes de la lige.

Bien souvent, au lieu d'être disposés sur une seule série longitudinale, on les voit disposés sur deux rangs juxtaposés; des cellules voisines qui les forment, l'une est en général plus développée que l'autre et se termine souvent en une pointe très acérée qui peut prendre des dimensions considérables (fig. 87 b.) 
A part ces poils physiologiques, nous trourons des tiges munies des mêmes papilles observées chez les feuilles.

Si nous comparons en effet l'épiderme de ces organes chez C. glauca avec celui de la lige, nous n'y remons aucune différence dans l'arrangement, la nature, la distribution de ces appendices; tout au plus constateronsnous sur la tige une petite diminution dans leur nombre. Il en est de mème chez C. Riparia.

Les cellules qui forment l'épiderme conservent la plupart du temps la mêne forme plus ou moins ovale ou arrondie. Parfois elle approchent de très près de cette dernière (C. montana), on bien, - surtout chez celles qui ont une tendance à former des papilles bouclées, c'est la paroi extérieure qui fait légèrement saillie, et s'épaissit de façon à donner au lumen une forme pyramidale (C. glauca). Mais le plus sourent ce sont les contours ovales, allongés et aplatis dans le sens de la tangente qui dominent.

Comme dans la feuille, ces cellules épidermiques sont susceptibles de silicification et peurent ètre munies de cònes, surtout en regard des revètements fibreux des faisceaux.

L'appareil stomatique présente le même aspect et la mème structure que celui de la feuille, c'est-à dire que chez les espèces où les stomates sont enfoncés sous l'épiderme, les cellules annexes faisant saillie au-dessus de l'ouverture, ces organes se présentent aussi cachés par les cellules épidermiques, lesquelles sont pourrues également de parois zigzaguées.

Le système lacunaire est représenté par des espaces de forme plus ou moins arrondie, irrégulière, placés en alternance arec les faisceaux, à la périphérie et séparés de l'épiderme par quelques couches seulement de lissu 
chlorophyllien. Parfois aussi, ces lacunes prennent un développement tel qu'elles refoulent les cellules vertes contre l'épiderme el les colonnes d'éléments sclérenchymateux (C. paludosa, C. vesicaria); dans d'autres cas par contre, elles sont à peine ébauchées, comme le montrent les parties supérieures de beacoup de tiges.

Faisceaux libéro-ligneux. - Ces faisceaux, composés sur le même modèle que ceux de la feuille, sont ici aussi collatéranx et fermés par une gaîne dont les éléments présentent des épaississements variables suivant les espèces: tantôt en effel on aura un épaississement général, intéressant à la fois toutes les cellules qui constituent la gaine, tantôt ces parois massives se trouvent localisées dans les éléments qui entourent le liber ou seulement dans deux ou trois cellules latérales placées en regard de la gaine parenchymateuse cellules aqueuses).

Leur arrangement et leur nombre varient suivant les espèces. Le plus souvent dans une lige parvenue à son entier développement, ils sont disposés sur deux rangs, l'un extérieur marginal, l'autre plus intérieur et sensiblement en alternance avec le premier. Dans certaines espèces enfin, on trouve encore un troisième el mème un quatrième rang. (C. Grayï., fig. 183). De tous ces faisceaux, il n'y a que ceux du rang marginal, qui présentent des revêtements mécaniques en colonnes, plus ou moins considerables suivant les espèces.

Au groupe de ceux qui ont les faisceanx disposés sur deux rangs se rattachent les formes suivantes:
C. Oederi
C. punclata
C. Baldensis
C. glauca (fig. 108)
C. riparia
C. sylvatica
C. hirta 
Nous avons au contraire un seul rang périphérique chez :
C. paniculata (fig. 15)
C. alpestris
C. leporina (fig. 62)
C. virens (fig. 4)
C. chordorthiza
C. distans (fig. 121)

Certaines espèces sont richement pourvues sous le rapport du tissu conducteur, d'autres le sont moins comparatirement à celles-ci. C'est ainsi que chez C. Grayï, les sections transversales peuvent renfermer jusqu'à 65 faisceanx cnviron; ce nombre descend ailleurs considérablement et l'on oblient alor's le chiffre approximalif de 40 (C. glauca), de 30 (C. paniculata) et de 15 (C. montana, ornithopoda). Ces nombres varient naturellement pour une mème espèce suivant l'endroit de la tige où la section a ćté faite ou le nombre d'anastomoses et de bifurcations qui se sont produites.

La structure intérieurc de ces faisceaux a la plus grande ressemblance avec celle des faisceaux foliaires. Dans le bois se trouvent les mèmes éléments, c'est-à-dire des raisseaux réticulés, annelés et plus rarement des vaisseaux spiralés.

Les gaines fasciculaires sont en tous points semblables à celles de la leuille pour une même espèce.

Parenchyme de la tige. - Ici encore grande analogie: sous l'épiderme sc trourent des cellules présentant entre elles de grands méats et appondues bout à bout, formant aiusi un véritable lissu spongienx (fig. 63, C. leporina). On y retrouve les mèmes bifurcations de membranes, ainsi que les cellules tannifères, lesquelles peurent ètre disséminées dans le tissu ou disposées symétriquement à grauche et à droite des colonnes de sclérenchyme (figures 108, 121, 132).

Les cellules à chlorophylle occupent toujours par rappor't au volume total de la tige un espace assez petit. Si 
on tire en effet une ligne passant par les extrémités internes des faisceaux libéro-ligneux du second rang, on aura ainsi la limite intéricure pour les cellules vertes, tout le reste du corps de la tige, si elle ne se creuse pas, est occupé par une sorte de moelle, dont les éléments incolores possèdent une forme arrondie, rendue plus ou moins polygonale par leur pression réciproque et sont pourvus à leurs jointures de petits méats intercellulaires.

Tous les faisceaux sont encore entourés de la mème gaine parenchymateuse incolore que nous avons déjà constatée dans les faisceaux foliaires.

Dans la plupart des espèces les tiges se creusent de bonne heure ; rares sont les cas (C. Buxbaumii) où elles restent pleines en lraut comme en bas. Nous pourrons en outre observer chez quelques-unes, directement sous l'épiderme une formation de collenchyme qui concourt avec les colonnes fibreuses des faisceaux à la solidité générale de la lige (C. Hordeïstichos).

Sléréôme. - Le stéréôme, lui, diffère quelque peu dans sa répartition de celui que nous avons étudié chez les feuilles. Il est formé sur l'une des extrémités du faisceau de colonnes épaisses plus ou moins longues, et sur l'autre, de groupes mécaniques arrangés en demi-lunes. Tels sont les revêtements des faisceaux situés à la périphérie : pour ceux qui occupent l'intérieur de la tige, nous avons en général inégalité de développement à l'une des extrémilès, c'est-à-dire que le revêtement présente sur ce côté un plus grand nombre de couches de cellules. Les deux portions de l'enveloppe fibreuse marchent à la rencontre l'une de l'autre et se soudent quelquefois, il en résulte que le faisceau peut se trouver complétement entouré par ses éléments mécaniques.

Cette disposition reste la même dans le bas comme dans le milieu de la tige; mais lorsqu'on s'approche de 
l'épi, on voit alors les faisceaux quitter la périphérie el se rapprocher. La tige devenant plus petite, les revètements fibreux se soudent entre eux par l'extrémité interne de façon à former une assise continue d'éléments mécaniques.

Chez C. leporina, cette disposition commence déjà vers le milieu el l'on peut voir que les petits faisceaux, alternant arec les grands, sont reliés entre eux par leurs extrémités internes, séparant ainsi d'une façon nelle et tranchée le tissu chlorophyllien du lissu médullaire (fig. 62).

Chez C. Grayi, le stéréòme prend un développement encore plus puissant. Ici, tous les faisceaux anastomosent leurs éléments mécaniques, de façon à former une masse sclérenchymateuse compacte, dans laquelle se trouvent enchâssées, comme de petites oasis, les lacunes entourées de quelques cellules assimilantes (fig: 182). Sur des coupes pratiquées encore plus haut lans la tige, on voit les faisceaux quilter pour la plupart - avec ou sans leur gaine fibreuse - leur position sous-épiderınique, s'anastomoser entre eux par la parlie externe de leur revêtement de manière à former une première assise continue d'élćments fibreux, puis aussi par la partie interne pour former une seconde zône de même tissu. Dans le bas de celte mème lige, au contraire, on voit les faisceaux marginaux, munis de leur stéréôme, s'étaler en éventail sous l'épiderme, el se rejoindre parfois de faisceau à faiscerı, formant ici encore une assise compacte interrompuc seulement çà et là par du parenchyme (fig. 183).

Il arrive encore que dans des tiges, un còté - probablement celui qui fail face au vent dominant ou au courant de l'eau, - présente un développement mécanique remarquablement plus complet que celui des autres côtés. Le tissu sclérenchymateux forme alors une masse com- 
pacte assez épaisse courant sous l'épiderme en plusieurs assises de cellules, tandis que sur les côtés voisins, il se laisse encore diviser en segments qui correspondent cliacun à un fáisceau.

Il existe de nombreux cas de structure mécanique puissante. Chez C vesicaria, par exemple, les tiges hiangulaires sont pourvues d'arêtes très développées el de faisceaux disposés comme d'ordinaire sur deux rangs. Mais au lieu d'avoir simplement une colonne d'éléments mécaniques formant le revêtement externe du faisceau et une gaine des mêmes éléments protègeant la pointe interne, nous les voyons - surloul chez les petits faisceaux qui se trouvent opposés à la base des arêtes, - former aussi des colonnes à cette pointe interne. Celles-ci se continueront dans le parencliyme et formeront une série de petits groupes mécaniques, qui souvent rejoignent les revêtements des faisceaux voisins en formant avec eux une sorte de pont.

En général le bas des tiges montre une structure moins forle que les parties les plus élevées du même organe.

Ceci s'explique facilement si l'on considère le fait que celle partie de la lige est entourée d'enveloppes nombreuses, consistant en gaines foliaires superposées qui sont elles-mêmes déjà pourvues des mêmes moyens de résistance que le limbe élirgi des feuilles. Ces gaines renferment en effet des faisceaux libéro-ligneux à colonnes sclérenchymateuses el des épidermes à parois externes épaissies.

Les pressions extérieures seront donc considérablement amoindries dans ces parties, d'où inutilité d'un système ınécanique très développé.

\section{B) Du Rhizome en général}

L`s conditions d'existence de lit tige souterraine aménent dans ses tissus de notables changements, sous le rapport de leur distribution et de leur nature. 
Mais ici aussi nous avons une forme cornmune qui sert de type et autour de laquelle viennent se grouper d'autres formes en somme assez peu différentes.

C'est d'abord dans l'aspect extérieur que les caractères varient: le genre Carex possède en effet-des rhizòmes dont la structure extérieure est déjà employée en systématique pour la détermination des espèces.

Ces rhizsmes peuvent ètre respiteux. c'est-à-dire se. présenter sous une forme plus ou moins noueuse où les direrses parties de la tige souterraine, restant courtes se sont soudées entre elles, en s'encherêtrant les unes dans les autres. fls peuvent être aussi traçants, c'est-à-dire formés de liges droites, régulières, pourvus d'entrenouds bien distincts el raỵonnant dans tous les sens.

Quant aux caractères intérieurs, ils présentent une plus grande variété.

Prenons un rhizome, comme celui figuré (fig. 139) et pratiquons-y une section transversale dans une partie qui ne soit pas trop noueuse; nous y verrons diffèrentes régions qui s'étagent comme suit:

Tout d'abord, à la périphérie, une couche de cellules pouvant ètre regardées comme épidermiques, en général peu différenciées par leur lorme de celles yui se trouvent all-dessous, si ce n'est qu'elles sont cutinisées. Ces cellules sont fortement reliées entre elles latéralement et par leurs parois externes le plus souvent zigzaguées, tandis que la plupart du temps, elles ont relativement peu de cohésion avec les éléments sous-jacents. Comme dans la tige aérienne, il peut arriver - surtout en regard des groupes de sclérenchyrae, que ces cellules deviennent le siège d'une silicification, laquelle n'est jamais aussi considérable que celle qui a lieu dans les parties correspondantes de la tige aćrienne.

Chez C. atrata et $C$. glauca, les cônes siliceux sont faciles à observer el assez communs (fig. 84 et 107). 
Outre cette silicification, la cellule épidermique peut devenir, ainsi que tout le tissu environnant, un licu de dépòt pour des matières huileuses, à l'instar des stomates dans les feuilles. Ces matières se présentent en goultelettes rondes, très réfringentes, se colorant en r'ouge foncé par la teinture d'orcanelte et réunies toujours par groupes dans un angle de la cellule ou éclıelonnées le long les parois (C. Schreberi, fig. 54). C'est à ces corps huileux qu'est due l'odeur particulière qu'exhalent certains rhizomes.

Sous l'épiderme s'étend le tissu cortical, lequel peutêtre divisé en deux. grandes parties principales: la plus extérieure, primitivement non différenciée de l'intérieure et formée d'éléments se cloisonnant tangentiellement dans ses premières couches, ne tarde pas à épaissir ses parois et à se lignifier plus ou moins. On obtient alor's un tissu sclérenchymateux, richement pourvu de ponctuations (C. montana, fig. 75), dont les cellules présentent la forme tabulaire allongée en coupe longitudinale el une forme polygonale en section transversale.

Les premières rangées de ce sclérenchyme ne présentent pas de niéats intercellulaires, mais à mesure qu'on s'approche du cylindre central, ceux-ci font leur apparition. Une autre forme d'épaississement et qu'on retrouve presque toujour's mélangée à celle dont on vient de parler, est la forme collenchymateuse (C. punclati, C. Baldensis).

La disposition de toutes ces couches cellalaires varic pour chaque espèce; c'est aillsi que pour C. glauca on n’aräa qu'une ou deux assises d'éléments sclérenchymatcux, le reste de l'anneau se composant de cedlules lignifiées, mais à membrines minces; tandis que chez C. montana ou chez C. punctata, ce tissu occupe la moitié des couches corticales. Dans ce dernicr cas cncore, il peut y aroir: prédominance dans cet anneaı des pre- 
mières assises sur les autres sous le rapport de l'épaisseur des parois cellulaires.

En général l'anneà sclérenchymateux n'est pas continu, on observe en effet à la périphérie, directement sous l'épiderme, des g̨roupes de cellules fibreuses à parois beaucoup plus massives et à petits lumens, serrées les unes contre les autres, se distinguant encore par la coloration spéciale qu'elles prennent aux réactifs ou par celle qu'elles ont déjà sur les coupes non'encore traitées.

Ces groupes sont disposés sur tout le pourtour à intervalles plus ou moins égaux, et ont par ce fait évidemment pour but de renforcer encore la résistance des Lissus périphériques en venant ajouter leur effet mécanique à celui de l'anneau sclérenchymateux tout entier.

La partie la plus interne de l'écorce est, suivant l'espèce, forméc de cellules arrondies, à parois minces (C. punclata, fig. 139 et C. montana), disposées sans ordre régulier, ou au contraire composée d'éléments à parois massives, très fortement poncluées (fig. 171, 172) et garnies de méats qui alteignent souvent des dimensions considérables, C. acuta, fig. 72, C. divulsa fig. 5).

Ces ponctuations sont quelquefois si proches les unes des autres, que les parois sur lesquelles elles se trouvent présentent l'aspect de chapelets dont les grains seraient fixés à une petite distance les uns des autres.

Suivant leur orientation elles apparaissent sous la forme de petits trous entourés d'un cercle qui les enveloppe un par un ou deux par deux (fig. 172, 171 a).

[Comparer entre elles les figures ci-dessus (C. provincialis ; fig. 143, C. pseudo-Cyperus, fig. 72 (C. acuta); fig. 69 (C. stricta), fig. 12 (C. vulpina); C oederi (fig. 130.)]

Chez quelques espèces, le tissu parenchymateux prend un aspect différent, lorsque le rhizome acquiert un certain àge; tantôt on a dè grandes lacunes plus ou moins 
quadrangulaires qui sont rangées régulièreınent en cercle sous l'anneau de sclérenchyme et qui prennent parfois un développement tel, que tout le tissu se résorbe, séparant ainsi l'écorce exlérieur'e du cylindre central; mais le plus souvent le développement de ces lacunes ne va pas si loin; il reste alors entre chacune d'elles, formant colonnes, des bandes de parenchyme à parois minces, plus ou moins larges, dirigées suivant le rayon, et qui relient l'anneau sclérenclyymateux au cylindre central. On a ainsi une tige demi-creuse portant dans les colonnes des faisceaux libéro-ligneux.

A ce groupe appartiennent les espèces suivantes:
C. riparia
C. brizoïdes
C. ampullacea
C. chordormiza
C. Schreberi
C. arenaria.

Dans d'autres groupes, on trouve ou hien un tissu làche, laissant entre ses éléments des méats intercellulaires en grand nombre et mal définis dans leurs contour's (C. hirta, C. Grayi), ou bien encor'e une résorption par files radiales partant du cylindre central et intéressant plusieurs rangées de cellules, de sorte que l'on obtient un parenchyme rayonné. Dans ce nombre se rangent entre autres:
C. stricta
C. maxima
C. Buxbaumii
G. vulgaris.

Enfin d'autres rhizomes, comme ceux des C. montana, distans. paraissent ne pas avoir de lacmnes dans leur parenchyme.

Chez certaines espèces, nous rencontrons des formes de rhizome qui différent plus du type habituel. Il en est ainsi par exemple, chez C. chordorriziza.

Nous voyons en effet dans les stolons de celte plante la structure de la tige aérienne conservée dans son ensemble à peu près. D'abord no'us n'avons pas de corps 
central, mais à sa place une lacune autour de laquelle vient se placer un rang de laisceaux. Dans le parenchyme cortical s'ouvrent d'autres espaces aérifères, lesquels partent aussi des faisceaux et vont aboutir à un anneau de sclérenchyme épais.

L'épiderme, chez cette espèce, se trouve aussi différer considérablement. Au lieu d'avoir, comme c'est le cas ordinaire, une assise périphérique de cellules tabulaires à parois minces, ses éléments revètent chez ce Carex des parois massives à l'extérieur, comme sur la tige aérienne. Ce n'est pas à proprement parler un véritable rhizome, mais une forme de transition vers la tige proprement dite.

Très souvent on observe le long des parois des cellules qui entourent les lacunes, dans le parenchyme en voie de résorption, des renflements plus ou moins considérables, semblables à des nodosités. Ces corps noueux, examinés à un fort grossissement, sont lormés cliez C. ampullacea (fig. 154), par des courbures de la membrane cellulaire. Ces courbures amènent la formation de zigzags irréguliers, de sinus plus ou moins profonds, qui, sous l'influence de la pression exercée sur eux par les tissus voisins, collapsent entre eux et viennent se souder intimément à la paroi commune (c). Cependant ${ }^{1}$ toutes ces nodosités n'ont pas la même origine, car on en voit qui résultent de l'application, par suite des mêmes pressions, d'un fragment de paroi rompue contre une autre paroi voisine, c'est le phénomène qu'on troure représenté dans la mème fig̣ure en $s, s^{\prime} s^{\prime \prime}$. Nous aurons l'occasion de revenir sur ces uodosités à propos de corps analogues qui se trourent dans l'écor'ce de la racine.

1 Le tissu cortical du rhizome de C. riparia présente aussi des nodosités semblables; mais leur forme est plus allongée et présente des étranglements loc:aux (fig. 150). 
6'

Nous avons vu que le tissu mécanique est ici représenté de différentes manières, d'abor'l par un anneau sons-épidermique de cellules à parois tantôt très ćpaisses, tantôt relativement minces, polygonales, dont les premières couches sous l'épiderme se cloisonnent tangentiellement, et sont toujours lignifiées; nous avons vu ensuite que ce tissu peut prendre facilement l'aspect collenchymateux et former une couche continue, laquelle peut pousser des prolongements vers le centre ou former des faisceaux courant parallèlement le long de la tige, comme le font les petits groupes scléreux à éléments massils disposés sous l'épiderme de distance en distance.

Outre ces différents moyens de résistance, il y en a encore un autre qui consiste dans la présence entre les parois contiguës de deux cellules, de méats locaux logés entre les deux membranes cellulaires. Ces méats - que nous appelons bifurcations, pour les distinguer des méats intercellalaires proprement dits qui se produisent aux points de jointure de plusieurs cellules - sont répandus sur la plupart des parois dans le tissu cortical interne, formant par places de longues séries de bifurcations appondues les unes aux autres.

Comme rôle à remplir, on ne peut guère leur assigner autre chose que l'aération et une résistance à opposer aux effets mécaniques. Il est mème à notre avis beaucoup plus probable que c'est surtout à cette dernière fonction que nous aurons à faire. Elles se rencontrent en effet de préférence chez les Carex aquatiques qui doivent évidemment présenter une structure plus forte leur permettant de résister avec facilité à l'action mécanique de l'eau et du vent. De plus elles apparaissent surtout sur les sections longitudinales, où elles sont orientées toutes d'une façon analogue, à savoir parallèlement à l'axe de la tige. 
65

Chacun sait aussi qu'un corps, tel qu'une paroi cellulaire, résultant de l'application l'une sur l'autre de deux membranes, présentera une résistance beaucoup plus forte au ployement, si dans son milieu il se forme un ou plusieurs espaces sur lesquels le collement n'a pas eu lieu et où se sont formés des vides intercellulaires arrondis. C'est en effet comme si l'en collait deux feuilles de papier l'une sur l'autre très intimément; ainsi disposée, lá nouvelle feuille double résultante sera, il est vrai, plus résistante, mais elle se pliera aussi facilement qu'auparavant sous une action mécanique quelconque. Il en sera bien autrement, si en quelques points de la surface, on a ménagé entre les deux feuilles des espaces remplis d'air formant vessies; en exposant le tout aux forces extérieures, on verra de suite que tout le système a acquis par ce fait une rigidité bealucoup plus grande et que pour vaincre celle-ci, il faudra un effort beaucoup plus considérable.

Cette structure à bifurcations n'est pas spéciale à l'écorce du rhizome, nous l'avons déjà observée chez les feuilles, d'une façon non moins frappante, nous la verrons reparaitre dans le parenchyme ligneux du cylindre central.

Cellules tannifères. - Comme les fevilles et les tiges aériennes, le parenchyme cortical et anssi le parenchyme ligneux à l'intérieur de l'endoderme présentent quelques cellules qui deviennent des réservoirs à tannin.

Ces cellules se distinguent par leur aspect incolore au milieu du tissu cortical souvent richement pourvu d'amidon et par leur grandeur plus considérable.

Traitées par les réactifs du tannin, le bichromate de polasse et le molybdate d'ammoniaque dissout dans le chlorure d'ammonium, elles présentent immédiatement la couleur caractéristique; les unes apparaissent rem- 
plies de précipité, les autres au contraire, qui se sont à moitić vidées, ne se colorent que partiellement. Pas plus que dans la feuille, on n'a affaire ici à des canaux continus ou à des séries de cellules appondues bout à bout, car en coupe longitudinale comme en coupe transversale, ces cellules tannifères se présentent isulées et avec la mêrne forme arrondie, parfois, il est vrai, quelıue peu allongée.

Elles existent en grand nombre dans les rhizomes des C. strigosa, distans (fig. 124) et C. punctata.

Outre ces cellules spécialisées en dépôt de tannin, le parenchyme cortical renferme aussi des faisceaux libéroligneux de structure collatérale, entourés toujour's d'une épaisse couche d'éléments mécaniques lignifiés. Nous aurons l'occasion d'en reparler à propos des faisceaux du cylindre central.

\section{Cylindre central}

Nous diviserons cette étude en deux parties: dans la première nous passerons en revue les différentes formes que présentent les éléments de l'endoderme, et dans la seconde nous étudierons le système conducteur, ses variations, ses rapports avec le tissu voisin et, d'une façon générale, la marche des faisceaux et leur passage du rhizome dans la lige aérienne.

Endoderme. - L'assise la plus interne du lissu cortical ou, si l'on veut, l'assise la plus externe du cylindre central, l'endoderme sépare d'une façon très tranchée les deux parties constitutives du rhizome.

Jans le jeune àge, - mais la chose est toujours tròs difficile à observer, - il offre sur les parois latérales de ses cellules, dans leur moitié interne, des plis qui forment 
des points obscurs (points de Caspary) et qui (fig. 101) les caractérisent comme tissu subérisé, analogue en tous points à celui de la racine.

Ce qui différencie plus spécialement l'endoderme chez les Carex, c'est l'èpaississement considérable dont sont susceptibles ses parois. Cet épaississement affecte toujour's la face interne des éléments; il s'étend aussi aux autres parois, mais on observe alors presque constamment que c'est sur le côté interne qu'il a été poussé le plus loin.

On aura donc, si nous considérons une cellule endodermique en coupe longitudinale, un corps allongé de forme tabulaire, dont les parois peuvent avoị la même épaisseur sur tout le pourtour de la cellule, ou bien, passant à l'extrême, présenter du côté du cylindre central, une épaisseur deux, trois, quatre et cinq fois plus considérable. Toules ces cloisons, les longitudinales comme les transversales, lesquelles sont le plus souvent obliques, sont richement sculptées de ponctuations très profondes.

La grande majorité des rhizomes montrent des endodermes massifs; quelques espèces seulement sont moins bien pourvues, telle est C. Schreberi, dont l'endoderme (fig. 55) est composé d'éléments tantôt ovales, tantôt pyramidaux de forme - celle-ci varie en effet pour un même rhizome, surtout s'il est cespiteux, suivant la partie sectionnée - et dont les parois présentent une épaisseur à peu près égale sur toutes les faces de la cellule. Il en est à peu près de même chez C. præcox (fig. 77) où nous avons un endoderme composé d'éléments arrondis.

Si nous passons à un degré un peu plus élevé, nous arrivons à Carex brizoïdes, qui présente peu de différence avec son voisin, C. Schreberi, puis à C. riparia (fig. 149) où les parois s'accentuent déjà beaucoup plus de façon à intéresser tous les côtés de la cellule. Nous arrivons ensuite à C. ampullacea (fig. 154), où la forme de l'élément 
68

devient parfois polygonale, à C. maxima (fig. 112) oủ les lumens sont pyriformes et à $\mathrm{C}$. distans, où nous trouvons une forme de croissant, analogue à celle de la fig. 133.

Celle dernière forme, qui peut devenir aussi celle d'un $U$, quand le grand axe de la cellule se trouve allongi en direction radiale, se rencontre dans la grande majorité des espèces.

Parmi les espèces ayant un endoderme en forme de croissant, nous avons :
C. punctata (fig. 133)
C. distans (fig. 123)
C. multiflora (fig. 68 b)
C. haldensis (fig. 164)
C. pseudo-Gyperus (fig. 143 a)
C. præcox (fig. 77) et beaucoup d'autres.

Dans les formes en $\mathrm{V}$, nous aurons :
C. hordeïstichos (fig. 180)
C. divulsa (fig. 5)
C. Schreberi (fig. 55)
C. muricata (fig. 6)
C. riparia (fig. 149)
C. alba (fig. 90)
C. depauperata.

Chez C. alba, nous trouvons déjà un endoderme si considérablement renforcé sur le côté interne que le lumen de ses cellules se trouve réduit de beaucoup.

La même chose a lieu, et d'une façon plus exagérée. encore, chez $\mathrm{C}$. acuta (fig. 71).

L'élément endodermique se rencontre plus rarement avec des contours arrondis, nous avons déjà cité le cas de C. præcox, nous ajouterons celui encore plus caractéristique de C. Grayï (fig. 184 a)

A l'endoderme est contigu une sorte de péricambium qui forme une couche d'épaisseur variable el dont les parois des éléments prennent diver's aspects, suivant qu'elles se sont lignifiées ou qu'elles se sont épaissies.

Le plus souvent, en effet, il se convertit en un anneau 
sclérenchymateux simple ou double (C. stricla, C. alba, fig. 90) contre lequel et dans lequel viennent s'appuyer les faisceaux libéro-ligneux (C. Grayï, fig. 110), C. acuta (fig. 7I) - - et dont les éléments de la couche la plus extérieure envoient des prolongements qui viennent s'intercaler entre les cellules de l'endoderme (fig. $110 a$ ). Les lamelles moyennes primaires dans ce tissu montrent par leurs réactions qu'elles sont fortement lignitiées.

\section{Tissu conducteur}

Les Cypéracées présentent une grande variété de structure dans leurs faisceaux libéro-ligneux; ces différences sont encore plus marquées dans le seul genre Carex.

En effet, nous avons ici suivant l'espèce des faisceaux à structure tantòt collatérale, tantôt concentriques, c'est-àdire que d'une part, on peut avoir des faisceaux oú les deux parties, bois et liber, se trouvent juxtaposées, en regard l'une de l'autre et enfermées dans une gaine de tissu sclérenchymateux plus ou moins épaisse, el que d'autre part, il existe une autre structure, ou c'est le bois qui entoure complètement la parlie libérienne, tout en restant lui-même enfermé dans une gaaine des mèmes cellules fibreuses. Bien plus, il est pas mal d'espèces où ces deux types de faisceaux, collatéraux et concentriques, se trouvent côte à còte (fig. 111), offrant la plupart du ternps, en section transversale, toutes les formes intermédiaires de passage. Ces dernières se rencontrent généralement à la périphérie du cylindre central, où les faisceaux s'anasto mosent sur une grande échelle en ne laissant presque pas de place au parenchyme.

Les faisceaux collatéraux du rhizome ressemblent en tous points à ceux que nous arons étudiés dans la feuille et dans la tige aérienne; nous ý voyons figurer les mêmes 
éléments : un phloëme composé de tubes criblés plus grands et de cellules annexes plus petites, un xylème plus ou moins allongé, et pourvu sur ses côtés de deux grands vaisseaux.

Les espèces de Carex qui possèdent de semblables faisceaux ne sont pas en majorité ; parmi celles-ci citons :
C. hirta
C. riparia
C. Grayï
C. ampullacea

On peut regarder le rhizome de C. Grayï, comme le type des rhizomes à faisceaux collatéraux, les stades de transition à la forme concentrique yétant très peu répandus et résultant d'anastomose et de fusion avec des faisceaux voisins (fig. 184).

Ces faisceaux sont disposés d'une façon irrégulière dans tout le tissu fondamental, mais de préférence dans les parties qui avoisinent l'endoderme, laissant ainsi toute la partie centrale occupée par du parenchyrne ligneux à méats intercellulaires souvent très prononcés et formé de cellules arrondies à parois minces (fig. $173 \measuredangle$ ).

Considérés en coupe longitudinale, les éléments toujours très ponctués de ce tissu fondamental présentent une forme allongée avec parois transverses, tantôt droites, tantôt obliques (fig. $173 a$ ).

Les plus intérieurs parmi ces faisceaux sont enfermés dans une gaine de cellules fibreuses, qui lcur est propre. Cette gaine présente en gènéral son maximum d'épaisseur sur l'extrémité interne, où elle se trouve formée de quatre à cinq couches de cellules épaissies, tandis que sur les côtés et à l'autre extrémité, ce nombre descend à trois el à deux.

Sous l'endoderme, par contre, toutes ces gaines s'anastomosent, de sorte qu'on obtient un anneau irrégulier zigzagué de tissu mécanique qui fait le tour du cylindre central à l'intérieur en suivant l'endoderme. Celte asso- 


\section{1}

ciation de tous ces éléments sclérenchymateux concourt à donner à l'organe total une faculté de résistance considérable.

Les faisceaux qui se trouvent cnfermés dans cet anneau, présentent au contraire de ceux qui sont situés plus à l'intérieur et qui ont une forme collatérale parfaite, des irrégularités dans la position de leurs deux parties constitutives.

La plupart du temps, dans les formes franchement collatérales, la partie bois ne prend pas un développement considérable; on n'a plus, comme dans la tige ou dans la feuille un xylème très allongé, où les vaisseaux sont rangés deux par deux; bien au contraire, le bois, ici très réduit, ne dépasse guère en élendue l'espace circonscrit par les gros vaisseaux, sans parler encore de cas assez nombreux où il n'existe qu'un seul de ces gros canaux.

L'espace silué entre ceux-ci est rempli par des éléments ligneux, éléments qui sont des vaisseaux spiralés ou annelés. Quant aux gros vaisseaux mèmes (vaisseaux scalariformes), ils sont ou directement contigus à la gaine fibreuse et entourés par des cellules à parois épaissies, aplaties tangenticliement, ou bien aussi complètement séparés de la dite gaine par un cercle complet de ces mêmes cellules aplaties.

Comme espèces présentant les deux formes, collatérale et concentrique, réunies dans le mèıne rlizome, à divers degrés de transition, on peut citer parmi celles qui ont les faisceaux en majorité collatéraux, C. hirta, et parmi celles qui, au contraire les ont sous la forme concentrique prédominante, C. maxima. Quelquefois mêrne celte espèce présente les deux formes opposées se touchant l'une et l'autre, comme dans la figure 111.

Les individus pourvus de faisceaux collatéraux présentent quelquefois des divergences notables suivant l'endroit du rhizome où l'on pratique les coupes. 
Il en est ainsi du C. dioïca, qui présente dans cet organe des parties traçantes régulières el d'autres noueuses dont la structure intérieure diffère considérablement. Dans ces dernières en effel, qui sont absolument souterraines, nous voyons tous les firisceaux disposer concentriquement leurs éléments, tandis que dans les slolons, nous avons une structure qui se rapproclse déjà beaucoup (cas analogue à celui de C. chordorrhiza) de celle de la tige aérienne.

Les faisceaux libéro-ligneux y sont collatéraux, serrés en uı rang, contre les cellules de l'endoderme et présentant ici et là une forme intermédiaire.

Dans tous ces faisceaux, les gaines fibreuses s'anastomosent aux deux extrémités, de sorte que l'on obtient deux cylindres creux concentriques formés de tissu mécanique et dont les contours sont ondulés.

Etudions maintenant les faisceaux concentriques. C'est la forme la plus commune et qui se trouve dans tous les rhizomes cespileux, comme aussi dans beaucoup de rhizomes réguliers (C. Schreberi), C. brizöides (fig. 158).

Ils diffèrent entre eux suivant les espèces, mais ces différences ne sont jamais que tout à fait secondaires.

C'est d'abord par leurs phloêmes qui, chez les uns (C. provincialis, C. Schreberi, C. brizoïdes) sont formés de tubes criblés el de cellules annexes présentant l'arrangement habituel typique (v. fig. 159) el qui chez les autres - si toutefois les mêmes cellules annexes y existent. - ne fournissent pas une différence aussi malquée entre les deux sortes d'éléments et où l'on a par conséquent tubes criblés et cellules annexes sensiblement de même gorandeur (C. glauca, C. rulpina. Ensuite vient le xylème, lequel entoure complètement la partie criblée dont il est séparé par du parenchyıne ligneux à parois minces.

C'est aussi ce même parenchyme qui sépare les pre- 
miers vaisseaux des éléments sclérenchymateus de la gaine, laquelle est constante pour ces faisceaux concentriques, mais qui varie suivant les espèces en forme el en épaisseur.

Cependant on observe des cas où les vaisseaux confinent directement à cette gaine.

Les premiers vaisseaux que l'on rencontre dans le xylème d'un faisceau présentent un grand lumen plus ou moins arrondi; ils forment un cercle simple ou double et peurent ètre par place directement contigus entre eux; mais le plus souvent il s'intercale entre leurs parois, rayonnant de la couche parenchymateuse qui les sépare du liber, des bandes de ce mème parenchyme qui peurent être simples, doubles ou triples.

Il existe toujours, le plus souvent sur un des côtés du faisceau, plus rarernent sur les deux, une bande de ce parenchyme qui est plus large que toutes les autres et qui semble mettre en relation plus intime le tissu fondamental avec le tissu intérieur du faisceau. Chez C. provincialis, et quelques autres, celte bande est à peine distincte de celles qui séparent entre eux les différents éléments du xylèıne.

Ceci s'explique peut-être par le fait qu'ici, la gaine ellemême remplit un peu le rôle de la bande large, les grains d'amidon qu'elle renferme prouvant qu'elle prend part aussi à l'acte de l'assimilation.

Chez C. stricta, les gros vaisseaux se trouvent sourent sur deux cercles concentriques séparés par du parenchyme ligneux. C. muricata présente lui aussi un déreloppement de vaisseaux semblable el qui peut nième aller encore plus loin. Nous avons en effet le plus souvent deux rangs, mais il peut arriver que l'on en rencontre trois, qui offrent une particularite en ce sens que tous les gros vaisseaux sont directement soudés les uns aux autres sans interposition de parenchyme ligneux. 
Laux ${ }^{1}$, dans un travail sur les rhizomes des Monocotylédones, a divisé ces différents organes, d'après la collatéralité, la concentricité des faisceaux ct la présence de lacunes dans le parenchyme cortical, en neuf types, dont le premier est représenté par $G$. chordorrhiza.

Or, d'après lui, ce Carex serait une espèce pourvue de faisceaux collatéraux normalement construits.

Ceci est vrai pour les rhizomes - si rhizome est un terme bon à employer dans ce cas - qui rampent sur le sol.

Ici, en effet, nous avons des faisceaux nettement collatéraux, des lacunes sous l'épiderme, lequel, comme nous l'avons déjà dit, est fortement épaissi.

Mais outre lit partie traçante, il y a un véritable rhizome. Celui-ci, comme tel, est souterrain el présente des faisceaux qui ne sont plus du tout collatéraux, mais au contraire nettement concentriques.

De ce qui précède et de ce que nous avons déjà dit de celte lige souterraine el de celle de C. dioïca (roir plus haut), il résulte que la forme de rhizome que cet auteur a prise comme type, n'est qu'une lorme de transition entre la lige aérienne et la tigge souteraine.

Le passage de la forme collatérale qui existe dans la tige, à la forme concentrique, qui est celle de la plıpart des faisceaux dans le rhizome, a lieu dans les nœuds de celui-ci, par suite de la juxtaposition à un autre faiscean intérieur d'un faisceau collatéral foliaire qui traverse le parenchyme cortical et se trouve toujours entouré d'une gaine fibreuse de forme arrondie.

Ceci dit, examinons ce qui se passe durant le trajet et l'entrée des faisceaux dans le cylindre central.

'Ein Beitrag aur Kenntniss der Leitbïndel im Rhizın Monocotyler P'tlanzen. Laux. Berlin 1887. 
Quelle que soit la nature de ceux-ci, on peut toujours observer que c'est au voisinage de l'endoderme qu'ils se trouvent en plus grand nomlsre.

Ceci provient du fait que, parmi les faisceaux foliaires, le médian pénètre en cet endroil dans le cylindre central jusque bien en avant à l'intérieur, pour se rapprocher ensuite de l'endoderme. Les faisceaux latéraux de la feuille suivent à peu près le même chemin, sans cependant s'enfoncer autant que le médian.

Il n'y a donc rien d'étonnant à ce qu'on trouve dans celte région un grand nombre de faisceaux serrés les uns contre les autres ou réunis par leurs gaines fibreuses.

Pour étudier les changements de forme que subissent les faisceaux, lorsque la tige, en changeant de milieu, perd sa qualité de tige aérienne pour devenir tige souterraine, il faut pratiquer une série de coupes à traver's les derniers entre-nouds, en commençant par le milieu d'un entrenœud normal bien développé.

Nous verrons que dans ces parties non régulières du rhizome, on peut constater au centre du cylindre central une tendance prédominante des faisceaux libéro-ligneux à la forme collatérale (ceci s'applique bien entendu au cas d'un rhizome à faisceaux collatéraux), tandis qu'à la périphérie plusieurs d'entre eux, en même temps qu'ils anastomosent leurs gaines fibreuses, passent au type concentrique plus ou moins parfait, pour reprendre ensuite leur première forme. Dans le cas des rhizomes à faisceaux concentriques, ces dernier's, une fois qu'ils ont revêtu cette forme, la conservent désormais durant toute leur course souterraine.

La transition délinitive de la forme concentrique à la forme collatérale a lieu seulement dans les derniers de ces entre-nœuds qui sont toujour's très courts.

En effel, si l'on suil dans un rhizome à faisceaux concentriques l'un de ceux-ci en partant du milieu de l'espace 
qui sépare deux nœuds consécutifs, l'on verra que déjà au passage du premier des nœuds rapprochés, la partie libé rienne du faisceau, quittant la position centrale, tend toujours plus à gagner le côté extérieur. Il y a en mêrne temps allongement de tout le système.

Cette disposition persiste à travers les entre-nœuds courts jusqu'au niveau du dernier nœud, où l'on voit le liber gagner décidément l'extrémité extéricure, pendant que deux gros vaisseaux se laissent déjà distinguer sur les côtés. Peu à peu il se produit une désagrégation dans les gaines fibreuses, puis chaque faisceau s'individualisant, se rapproche de l'épiderme où l'on commence à voir plusicurs d'entre cux oceuper à la périphérie la position qu'ils doivent avoir désormais.

L'endoderme qui jusqu'alor's présentait des éléments épaissis d'aspect particulier et formait un cercle autour du cylindre central, cesse d'apparaitre et à sa place se montrent des éléments prosenchymateux lignifiés analogues à ceux des gaines fibreuses.

L’anneau sclércnchymateux du rhizome persiste encore, mais peu à peu, il n'est plus représenté que par une couche de cellules lignifiées à parois minces, courant sous l'épiderme.

Bientôt aussi apparaissent les lacunes à la périphérie sous l'anneau. Chacune d'elles est séparée par une mince bande de parenchyme qui renlerme un faisceau dont les éléments de la gaine montrent à l'extrémité extérieure une tendance marquée à se relier aux cellules de l'anneau sclérenchymateux. Cette jonction a lieu enfin et l'on voit alor's les laisceaux de la périphéric toucber à l'épiderme par leur's revêtements, tanılis que l'anneau se trouve interrompu en regard des lacunes par le tissu chlorophyllien. Il ne subsiste bientôt plus que sous la forme de revêteinents mécaniques pour les faisceaux, et dans les parties 
qu'il occupait primitivement, il se troure remplacé par des cellules épidermiques qui prennent quelquefois la forme collenchymateuse.

Remarquons enfin pour terminer le sujet, que les faisceaux libéro-ligneux du rhizome diffèrent de ceux de la tige aérienne en ce qu'ils n'ont pas de gaine fasciculaire nettement différenciée par sil forme des éléments fibreux constituant le revêtement; où en d'autres termes, en ce qu'ils ne possèdent pas, à leur pourtour, de gaines dont les cellules, très épaissies et disposées sur une couche, enveloppent les deux parties du faisceau, tout en les séparant franchement des éléments fibreux qui les protègent.

\section{ÉTUDE DE LA RACINE}

Disons tout d'abord un mot de l'état de l'organe à ses premiers débuts.

Si nous prenons une loute jeune racine, et si nous y faisons une coupe transversale, nous trouverons le tissu déjà nettement différencié en plusieurs régions.

C'est ainsi, par exemple, que nous aurons à lạ périphérie deux rangées de cellules à grands lumens et à parois minces, allongées - surtout celles du rang extérieur - dans le sens du ravon et se cloisonnant tangentiellement. Celles du rang interne sont déjà plus arrondies, moins comprimées, faisant ainsi transition avec la zône plus intérieure qui leur succède et qui est formée de cellules polygonales plus petites, à parois plus fortes, disposées sur quatre ou cinq courhes d'épaisseur et présentant ici et là un élément plus grand que tous les autres. Toutes ces cellules ne laissent entre elles aucun méat. 
Le tissu sous-jacent est constitué par des éléments plus ou moins ovales, arrondis, disposés en séries radiales régulières, diminuant de volume en s'approchant ver's le cylindre central, et laissant entre eux - aussi en rangées régulières radiales - de grands méats intercellulaires, qui ont la forme de losanges parfaits. Au voisinage de l'endoderme, ils épaississent quelque peu leurs parois, formant sur deux, el parfois sur plusieurs conches d'épaisseur, un collier de cellules quadrangulaires dont les méals vont en se prononçant toujours davantage.

L'endoderme vient alors terminer ces séries radiales, mais ses éléments ont encore la même épaisseur de parois. Ils diffèrent cependant déjà du tissu environnant par leur forme plus allongée dans le sens du rayon et par les points obscurs caractéristiques qui sont 'quelquefois assez apparents.

Suivant l'espèce, l'endoderme confine sur tout son pourtour à une assise péricambiale non encore lignifiée, interrompue ou non par les raisseaux primaires qui sont dejà fortement lignifiés et donnent, lorsqu'on les traite par la fuchsine ammoniacale, ou la phloroglucine, une belle coloration rouge. Ces vaisseaux primaires sont les seuls éléments qui, dans ce jeune stade, présentent cette coloration.

Peu à peu de son côté, le parenchyme du cylindre central acquiert de l'épaisseur dans ses parois. Il se lignifie aussi, en même temps que celui de l'écorce qui commence à montrer une distribution spéciale, par laquelle certaines de ces rangées radiales allongent leurs éléments dans le sens de la tangente, tandis que d'autres persistent dans leur forme primitive ; tout cela, de manière à donner enfin au tissu cortical moyen, l'aspect d'une toile d'araignée, aspect qui se retrouve presque constamment dans les racines des Caricinées (fig. $114 A$ ). 
Examinons maintenant de plus près la partie corticale et le cylindre central dans une racine qui a atteint son développement complet.

\section{Tissu contical}

La première assise est formée de cellules allongées dans le sens du rayon, à parois minces et dont plusieurs se développent en poils (fig. 66, 251) qui s'allongent quelquefois en longs boyaux et sont toujours unicellulaires, malgré certains épaississements ou dépôts de protoplasme desséché dans leur intérieur que l'on peut prendre à tort pour des cloisons transversales.

Parfois ils prennent une forme de massue, mais toujours d'une façon peu accentuée.

Cette assise pilifère n'a le plus souvent qu'une très courte existence ; en effet, on la voit prendre bientôt une couleur jaunâtre et se dissocier peu à peu pour disparaitre tout à fait, lor'sque les cellules des autres tissus ont achevé leur développement.

Chez certaines espèces (C. resicaria), les cellules périphériques (épidermiques, si toutefois ce terme peut ètre employé) augmentent d'épaisseur èt, en persistant, font corps avec l'anneau sclérenchymateux.

L'assise qui leur succéde vers l'intérieur, formée de cellules hexagonales à parois d'égale épaisseur, parait dans bien des cas subir le même sort; dans d'autres au contraire, on voit ses cellules, d'incolores qu'elles étaient d'abord, prendre aussi une couleur jaunâtre, persister et se subérifier de manière à former un tissu protecteur aux assises plus internes, à mesure que la couche pilifère disparaìt.

Aux couches ci-dessus, plus ou moins dissociées, succède une autre dont les éléments cloisonnés obliquement 
se colorent en rouge par les réactifs de la lignine, lorsque la racine a acquis un certain âge.

A mesure qu'on pénètre plus dlans l'intérieur, ce tissu présente des parois plus épaisses, de sorte qu'on a folmation l'un anneau de sclérenchyme dont les couches internes sont pourvues de cellules plus petites, mais plus fortes de parois, ct dont l'épaisseur totale comprend quatre à cinq rangées de cellules.

En continuant notre marche vers l'endoderme, nous pourrons constater que les éléments sont encore pourvus de parois solides, parfois collenchymateuses, mais non plus lignifiées. Cette sorte de sclérenchyme secondaire présente la même épaisseur que le premier.

La différence de nature qui existe entre les cellules qui remplissent les fonctions d'épiderme el l'assise sous-jacente des éléments hexagonaux se distingue facilement par le chlorure de zinc iodé. Les premiers prennent une couleur brune, tandis que les seconds deviennent jaunes.

On peut en outre constater dans certains cas une gélificalion plus ou moins complète des cellules épidermiques.

Le tissu cortical peut être divisé en tissu cortical externe et tissu cortical interne; tous deux se distinguent facilement par leur aspect différent.

La première partie se laisse discerner aisément par l'absence complète de méats intercellulaires; les cellules y sont de plus disposées sans ordre régulier.

Dans le tissu cortical interne (fig. 85, 46, 156) au contraire, elles sont disposées en files radiales, régulières, laissant entre elles des méats intercellulaires.

Dans chaque file, la cellule la plus interne, c'est-à-dire celle qui est contiguë à l'endoderme est la plus petite, la suivante est déjà plus grande et ainsi de suite en augmentant de diamètre du centre à l'extérieur.

La couche corticale interne est d'ordinaire du double 
ou du triple plus épaisse que l'externe. Ses éléments se divisent par des cloisons tangentielles, centripètes, ce qui maintient l'ordonnance régulière du tissu.

\section{IFrohifieations}

Lés deux tissus qui forment l'écorce ne persistent pas longtemps dans cet état, où ils ne sont différenciès l'un que par le manque, l'autre que par la présence de méats intercellulaires en files radiales.

Dans le tissu périphérique, il se forme, comme nous l'avons vu, un anneau silérenchymateux el parfois du collenchyme.

Dans la zone interne, la partie qui s'épaissit est au contraire celle qui touche au cylindre central. On observe en effet que les couches cellulaires immédiatement en rapport avec l'endoder'me prennent une forme ovale très accentuée et des parois plus massives dont l'épaisseur augmente a mesure qu'on approche du centre. Au contraire, en marchant vers la périphérie, on voit les cellules de ces files radiales diminuer et de force et de diamètre, de façon que dans la partie supérieure de celte zone interne, nous n'ayons plus que des membranes très minces, qui finissent par se résorber dans les vieilles racines, laissant le sclérenchyme former une enveloppe ou fourreau au corps central.

Cette résorption atteint aussi dans certains cas la partie la plus interne de l'écorce sans jamais cependant l'envahir tout entière; on remarque en effet toujours que l'endoderme reste protégé à l'extérieur par une ou deux rangées des cellules ovales ci-dessus mentionnées qui sont devenues plus ou moins brunâtres.

Lorsqu'on n'a pas résorption du tissu dans le parenchyme moyen, on a alors formation d'un tissu spécial 


\section{2}

formé d’une part par de grandes cellules (fig. 114 A) à parois minces, dont le grand diamètre est dirigé tangentiellement (a), et dont les parois parallèles à ce grand diamètre forment une sorte de pont entre des rayons de cellules rondes $(b)$ beaucoup plus petites qui sont restées en files radiales tantôt sur un rang, tantôt sur deux, et qui simulent assez bien les rayons d'une roue.

Nous avons donc ainsi un tissu en toile d'araignée formé d'une part de séries radiales de cellules reliant entre eux le cylindre et le sclérenchyme cortical, et d'autre part de membranes cellulaires, courant parallèlement entre elles et tangentiellement par rapport au corps total de la racine (C. Buxbaumii, fig. 67), C. Davalliana (fig. 46). Cette structure est très apparente chez $\mathrm{C}$. maxima (voir fig. $114 \mathrm{~A}$ ).

Elle se trouve de plus dans toutes les espèces ici étudiées, mais plus ou moins modifiées ou altérées par les pressions et résorptions qui se produisent dans la racine.

L'anneau sclérenchymateux peut, lui aussi, subir de pareilles modifications et présenter dans beaucoup de cás des couches internes collapsant entre elles, de manière à former un tissu compact, où les lumens des cellules, plus ou moins oblitérés, ne sont plus représentés que par des fentes allongées dans un même sens.

Nodosités. - En même temps que se forme le tissu en toile d'araignée, apparaissent, sur les parois des cellule étirées tangentiellement, des corps analogues à ceux que l'on a observés déjà chez quelques rhizomes.

Ces corps sont des nodosités qui souvent se montrent comme des bifurcations survenues à l'intérieur des membranes (C. paniculata, Davalliana (fig. $4 T$ ) el C. maxima fig. $114 A$ ).

Sur les coupes longitudinales, ces nodosités offrent sensiblement la rnême forme, toutefois un peu plus globuleuse. Dans d'autres cas, elles montrent une origine ana- 
logue à celle des mêmes corps qui se trouvent chez les rhizomes, oủ l'on a affairc à des plissements survenus dans les parois par suite de la pression des tissus environnants, plissements qui se sont plus ou moins rompus et dont la partie détachée estvenue se souder à la paroi qui la portait.

D'après Klinge ${ }^{1}$, on doil l'origine de ces nodosités à un collapsus des parois dans les cellules du tissu cortical moyen. Cet auteur est parvenu à distinguer dans la racine d'une Cypéracée non déterminée quant au genre auquel elle appirtient, non seulement les membranes tangentielles collapsées, mais encore les cloisons radiales, qui figurent en traits fins, noir's, au milieu de la nodosité.

Ce serait donc toute la paroi d'une cellule qui, en collapsant avec sa voisine, amènerait la formation de ces corps. Les cellules du parenchyme cortical nayant entre elles qu'une faible liaison, leurs parois se trouvent par suite de l'accroissement de la racine, allongées tangentiellement, et sous l'effet de cet allongement, elles se trouvent rapprochées les unes des autres, plus ou moins intimément. Mais, d'autre part, les cloisons radiales échappant au colIapsus, empêchent en certains endroits les deux parois de s'appliquer entièrement l'une sur l'autre, de sorte qu'on obtient une nodosité, laquelle laisse apercevoir on son milieu un trait noir le plus souvent courbé, reste de la cloison radiale primitive, ou bien ne laisse plus rien voir, et alors la nodosité prend l'aspect d'une bifurcation de membrane plus ou moins régulière de contours (fig. 114a) (a-f).

Parmi les Carex étudiés ici, il faut citer comme présentant ce phénomène d'une façon bien évidente : C. Davalliana (fig. 47), C. paniculata, el surtout C. Baldensis (fig. 167).

'Klinge. Vergleichende histolog. Untersuchung der Gramineen- und Cyperaceen Wurzeln. Mémoires de l'Acad. Imp. des Sciences de SaintPétersbourg. VIIe série, t. XXVI. 
Non seulement ce collapsus peut être tangentiel, mais il peut encore intèresser les parois radiales des cellules qui forment les rayons de la roue. On aura donc modification dans l'arrangement des tissus (C. maxima). On ne verra plus alor's autant de ces séries de cellules rondes, disposées en rayons. De celles-ci, les unes auront persisté, tandis que les autres seront remplacées par une ligne zigzaguée, à chaque courbure de laquelle viendra s'attacher alternativement de chaque côté, un des filaments tangentiels pourvu de nodosités (C. arida).

Les nodosités se trouvent sur les coupes longitudinales à des places déterminées, à intervalles égaux qui correspondent assez bien aux points de jonction des cellules primitives; ce fait confirme donc leur origine. Dans beaucoup de racines, il n'est plus guère possible de retrouver cette régularitć dans la distribution des nodosites, l'organe ayant été appelé à subir des changements de forme, des pressions et des déchirements en grand nombre.

Dans le tissu qui entoure l'endoderme, tissu qui est formé en général de cellules rondes ou ovales à parois épaisses, on peut trouver aussi des nodosités, mais elles présentent un aspect différent (fig. 8) provenant du fait que les parois qui les portent, sont beaucoup plus épaisses. Dans tous les cas, il est à remarquer que les deux ou trois couches qui entourent l'endoderme restent toujours étrangères à la formation de corps semblables. Ces nodosités proviennent sans doute de plissements, comme dans fig. 150, 154.

Doit-on voir dans la présence de ces nodosités une mesure de protection, en vue de rendre les parois résorbées plus solides et utiles à quelque chose en les faisant collaborer à la consolidation de l'organe? Il est en effet possible, qu'ainsi pourvus de nodosités, ces filaments qui représentent une double membrane, exigent 
85

pour la rupture un plus grand effort. Mais cependant, il semble que leur effet doit être bien secondaire, comparativement à celui de l'anneau sclérenchymateux d'une part et à celui des cellules si puissantes qui composent l'endoderme d'autre part.

\section{Endoderne}

Il en est de l'endoderme de la racine comme de celui du rhizome. Nous y trouvons la même variété d'aspect et les formes en $\mathrm{U}$ et en croissant pour les éléments qui le composent.

La grande majorité des endodermes présentent la première de ces formes, et pour citer des exemples, nous dirons qu'il en est ainsi chez :

C. alba (fig. 91 a)

C. Schreberi (fig. 56)

C. leporina (fig. 64)

C. distans (fig. 126)

C. punctata

C. Oederi (fig. 137)

G. divulsa (fig. 8)

C. pracox (fig. 78)

C. muricata

C. depauperata (fig. 129)

C. vulpina (fig. 13).

Comme précédemment, nous pourrons avoir aussi un égal épaississement sur tout le pourtour de l'élément endodermique, C. arenaria (fig. 162), C. præcox (fig. 78), C. distans (fig. 126), C. Schreberi (fig. 56) on bien cet épaississement n’intéressera que la moitié interne, laquelle fera alors un contraste marqué avec les parois antérieures comme chez:

G. Buxbaumii (fig. 67), C. multiflora (fig. 58)

C. Oederi (fig. 137).

D'autres fois l'épaississement que subissent les cellules sur leur face interne devient très considérable el donne 
au lumen une forme de poire; c'est le cas entre autres pour: C. Buxbaumii (fig. 67)

C. Oederi (fig. 137)

C. atrata (fig. 85)

C. maxima (fig. 112).

Tous ces éléments richement pourvus de ponctuations, communiquent par elles avec le cylindre central et les premières couches corticales. Quelques-uns d'entre eux montrent quelques jolis exemples de ponctuations ramifiées (C. acuta fig. 73).

Dans leur état jeune, ils laissent apercevoir assez facilement les points de Caspary qui sont situés, comme dans les rhizomes, sur la moitié interne des parois latérales.

Toutes ces parois sont alors si minces qu'on les reconnaît à peine au milieu du tissu cortical yui les environne.

\section{Cylindre central}

Le parenchyme ligneux se distingue dès les premiers temps par la forme polygonale de ses cellules qui font brusque contraste avec celles de l'écorce interne où l'on a une forme carrée et une disposition par séries radiales.

Le jeune faisceau, lui aussi, consiste en cellules à parois minces, hexagonales, dont la forme peut se modifier et devenir parfois cubique.

Les tissus du cylindre central s'accroissent rapidement et présentent des réactions différentes avec les divers réactifs colorants. C'est ainsi que le centre du corps occupé par le parencliyme médullaire, se colore en violet avec le chlorure de zinc iodé, tandis que les autres parties prennent une couleur jaunâtre différente.

Considéré dans son ensemble, il présente trois parties principales : 
$1^{\circ}$ La zone péricambiale,

$2 \circ$ Les lames vasculaires,

$3^{\circ}$ Le parenchyme médullaire.

Nous allons étudier sépirément ces diverses parties.

Zòne péricambiale. - Elle est en général simple, c'est-àdire formée d'une seule assise d'éléments, continue, ou (suivant les espèces et dans la majorité des cas) interrompue par les vaisseaux primaires qui viennent se placer directement sous l'endoderme, lignifiée ou non (comme chez C. arenaria), présentant quelquefois en regard des lames vasculaires une ou deux cellules dont le diamètre dépasse celui des autres éléments. Dans d'autres cas au contraire, la cellule comprise entre les vaisseaux el l'endoderme se trouve aplatie el prend ainsi un lumen plus étroit.

Entre ces deux formes où le péricambium constitue une zone continue, et celle où celle même assise est constamment interrompue par les vaisseaux primaires, il y a des formes intermédiaires dans lesquelles on rencontre des faisceaux confinant directement à l'endoderme et d'autres qui en sont séparés par l'épaisseur du péricambium.

Chez certaines espèces l'une ou l'autre de ces disposilions pourra prédominer; on pourra ainsi avoir une espèce dans laquelle il y a prédominance des endroits où le péricambiurn est coupé, et une autre où ces interruptions ne seront pour ainsi dire qu'une exception. On trouvera même des racines chez lesquelles il $y$ a attenance de groupes-xylèmes contigus à l'endoderme avec d'autres groupes non contigus.

Les individus qui présentent un anneau péricambial continu ne sont pas nombreux, parmi ceux que nous avons rencontrés, nous pourrons citer : 
C. arenaria (fig. 162) C. glauca

C. humilis

C. alba (fig. 91 a)

C. depaupérata (fig. 129) C. alpestris.

D'autres enfin, comme C. provincialis, C. nitida, C. muricata, C. divulsa, C. præcox, présentent un anneau péricambial interrompu seulement en un ou deux endroits tout au plus.

Chez C. atrata, C. nigra, C. Baldensis, l'interruption régulière par chaque vaisseau primairẹ devient au contraire la règle.

Tissu conducteur. - Ce tissu présente chez les Carex la disposition normale des racines de Monocotylédones.

Le nombre des faisceaux ligneux qui alternent contre la zone péricambiale varie beaucoup d'espèce à espèce et d'une racine à l'autre, suivant la grosseur de la racine et le diamètre du corps central. C'est ainsi que chez une racine jeune de $\mathrm{C}$. arida, nous aurons seulement quatorze vaisseaux primaires el vingt-huil dans une autre racine plus âgée de la même plante.

L'arrangement des faisceaux ligneux en séries radiales centripètes varie aussi suivant les espèces: tantôt leurs vaisseaux sont disposés régulièrement en lames continues, tantôt ils sont placés sans ordre les uns au-dessus des aulres et interrompus par du tissu conjonctif.

Le plus souvent, le xylème ne correspond pas directement avec les grands vaisseaux centraux, mais on a deux groupes xylématiques correspondant à un gros vaisseau, et alternant en même temps avec leux faisccaux libériens.

Si nous observons en coupe longitudinale les faisceaux ligneux qui composent le cylindre central, nous constaterons, comme l'a déjà fait aussi Klinge, qu'ils renferment tous les genres possibles de vaisseaux (ponctués, réliculés, scalariformes) à l'exclusion des vaisseaux annelés et spiralés. Chez C. Davalliana (fig. 187, 4), nous 
trouvons entre autres de fort jolis spécimens de vaisseaux finement réticulés, dont le réseau semble formé par deux spires très serrées et très fines marchant parallèlement à la rencontre l'une de l'autre en se coupant un grand nombre de fois.

Examinons maintenant les relations qui existent entre la tige et la racine, puis entre celle-ci et les radicelles.

L'insertion des racines sur le rhizome a lieu suivant la règle habituelle, par développement endogène, et comme les faisceaux du rhizome sont à la périphérie serrés les uns contre les autres, il s'en suit que l'espace qu'ils laissent entre eux est le plus sonvent très étroit, ce qui facilite l'insertion d'une racine. On voit en effet sur des sections longitudinales passant par le point d'insertion, les vaisseaux des deux faisceaux qui limitent l'espace rhizogène s'infléchir, puis pénétrer dans le mamelon conique qui forme la première ébauche de la future racine.

Peu à peu ce mamelon s'allonge, perce les lissus qui lui sont superposés, les digère et arrive enfin au nireau de l'épiderme.

Pour l'insertion des radicelles sur la racine-mère, nous arons une processus analogue; seulement elle peut avoir lieu de deux inanières différentes suivant quc l'anneau péricambial est ou n'est pas interrompu par les vaisseaux primaires du xylème.

Les cas normaux sont ceux où les radicelles prennent naissance de cellules situćes dans la zône péricambiale, en regard des raisseaux du bois. Ce cas se rencontre chez les Carex pourrus d'un péricambium continu, mais l'on sait que la plus grande partie des espèces ne répondent pas à cette structure normale. Comment aura donc lieu l'insertion des radicelles dans les espèces dont 
le péricambium se trouve interrompu par les vaisseaux primaires?

C'est ce que nous montrent les coupes transversales laites sur les racines de Carex tels que C. atrata et C. ornithopoda (fig. 185).

Ces coupes prouvent que celle insertion, yui ne peut avoir lieu ris-à-vis d'un vaisseau primaire, puisqu'il n'existe pas en cet endroil d'arc rhizogène, s'effectue entre deux lames vasculaires en regard des vaisseaux libériens où l'assise péricambiale existe toujour's (figg. 185). La sortie des jeunes radicelles, qu'elle ait lieu vis-a-vis ou en dehor's du vaisseau primaire, s'accomplit par digestion, comme on l'a vu plus haut à propos des rapports qui existent entre la tige souterraine et la racine.

L'insertion d'une radicelle amène dans les tissus environnants des modifications considérables. Chez C. muricata, on peut en effet observer facilement le fait qu'au lieu de se résorber, comme cela a lieu d'ordinaire, le parenchyme cortical silué entre l'anneau sclérenchymateux el l'endoderme, épaissit au contraire considérablement les cloisons de ses cellules au voisinage du point d'insertion, de sorte que la jeune radicelle se trouve protégée par un manteau d'éléments à parois fortes, semblables à ceux qui composent les premières couches corticales au pourtour de l'endorlerme.

Si nous récapitulons ce qui vient d'être dit de la structure interne des racines dans le genre Carex, nous voyons que pour cel organe, il y a aussi un plan-type génćral, d’après lequel il est bâti el qui, de même que pour la tige, se retrouve dans toutes les espèces sans présenter de divergence importante.

En oulre, - el toujour's en r'ésumant, - nous pourrons dire que la racine des Carex est une racine normale 
qui ne présente qu'une seule particularité, qu'elle partage avec les Graminées, à savoir d'être munie d'un péricambium qui, la plupart du temps est interrompu par les vaisseaux primaires du xylème.

C'est là le seul point capital intéressant qui nous soit offert par l'organe que nous venons d'étudier.

\section{PARTIE SPÉCIALE}

Les espèces dont la structure anatomique a été prise pour base de ce travail sont les suivantes:

C. muricata

C. divulsa (C. virens)

C. vulpina

C. paniculata

C. Davalliana

C. dioïca

C. chordorrhiza

C. Schreberi

C. brizoïdes

C. leporina

C. Buxbaumii

C. stricta

C. vulgaris

C. acula

C. montana

C. precox

C. humilis

C. alpestris

C. resicaria

C. hirta

C. baldensis
C. nigra

C. atrata

C. or'nithopoda

C. digitata

C. alba

C. nitida

C. glauca

C. maxima

C. strigosa

C. distans

C. depauperita

C. punctata

C. Oederi

C. sylvatica

C. pseudo-Cyperus

C. riparia

C. paludosa

C. ampullacea

C. arenaria

C. Grayi

C. provincialis

C. hordeïstichos 
92

Avant de passer à l'étude détaillée des espèces ci-dessus indiquées, étude qui aura surtout pour but de rechercher quels sont les caractères anatomiques qui peuvent servir à des groupements systématiques, il est bon, retenant ce qui a été dit plus haut des organes de végétation en général, de ne pas perdre de vue les quelques considérations suivantes :

$1^{\circ} \mathrm{La}$ structure anatomique d'une plante n'est pas un effet du hasard; elle doit correspondre à des exigences physiques et physiologiques.

(En parlant de la distribution des élérnents mécaniques, des cellules silicifères, secrétrices, etc., nous avons vu ce que l'on entend par là et en quelle mesure les Carex y répondent en général).

20. D’après ce qui précède, nous devoris trouver dans la structure anatomique une analogie de disposition ou des fonctions succédanées chez les formes proche parentes. (Nous verrons par ce qui suit que les espèces formant un groupe systématique, forment aussi un groupe naturel que les caractères anatomiques viennent le plus souvent confirmer).

$3^{0}$ Trouve-t-on chez des espèces systématiquement très éloignées des ressemblances ou des identités dans les caractères anatomiques, cela pourra signifier identité des conditions extérieures de végélation.

$4^{\circ} \mathrm{Il}$ en résulte que seuls, donneront des caractères employables systématiquement, les faits qui ne reposent pas sur des adaptations ou qui peurent être eux-mêmes r'egardés comme tels.

$5^{\circ}$ Les caractires anatomiques qu'on ne pourra employer dans un but systénatique seront:

a) la distribution des éléments mécaniq̣ues, sitòt qu'il s'agira d'espèces qui ont des fonctions mécaniques différentes à remplir'. 
b) la formation des stomales.

c) la consolidation des feuilles par un renforcement de l'épiderme, par des plissements ou l'intercalation d'éléments mécaniques dans le tissu fondamental.

d) la présence d'huile, de silice, de tannin.

Par contre, si nous trouvons des caractères qui ne puissent être reconnus comme provenant d'une adaptation, ces caractères permettront de parler de véritable parenté, aussi bien que ceux donnés par les fleurs. Mais en faisant l'étude comparée des espèces ci-dessus, nous verrons que si l'on peut répondre affirmativement sur quelques points, il en est beaucoup d'autres sur lesquels il est impossible de se prononcer, à cause de l'infinie variété des formes.

On ne peut donc qu'ètre d'accord avec MI. Schwendener, lorsqu'il dit que la véritable valeur systématique des caractères anatomiques ne peut être jugée qu'après une étude approfondie des différentes variations locales d'une seule et même espèce. $0 r$ ces variations ètant très nombreuses chez les Carex, il faudra user d'une extrême prudence dans les conclusions.

Procédons maintenant à l'examen des espèces proposées, et voyons quels sont les résultats que nous en pourrons tirer.

Carex Muricala L. (Reich. I. con. VIII, 561)

A cette espèce se rattachent plusieurs autres formes ou variètés, telles que C. divulsa, Good et C. virens, Lam. Nous étudierons d'abord la structure anatomique chez C. divulsa, puis nous la comparerons à celle du type C. muricata.

\section{Divulsa (Good.)}

Epiderme compósé comme habituellement de cellules 
arrondies et plus grandes sur la page supérieure, ovales sur la page inférieure, à parois externes plus épaisses et muni de poils en forme de crochets sur le bord de la feuille. Surface lisse quelque peu bosselée en regard des cloisons transversales dans les cellules de l'èpiderme supérieur (coupe longitudinale).

Cônes siliceux dìns les éléments épirlermiques qui confinent au tissu mécanique, lequel est représenté par des groupes de cellules sclérenchymateuses situés sous l'épiderme supéricur en regard de chaque faisceau libéroligneux et séparés de lui par une colonne de cellules aqueuses, qui se continuent aussi tout autour du même faisceau (voir fig. 1).

Les cellules de plissement se présentent ici disposées sur un seul rang et faiblement développés en proportion des autres cellules de l'épiderme ; leurs parois extérieures sont l'ecouvertes d'une épaisse culicule prćsentant de nombreuses rides (fig. 2).

Faisceaux disposés en aiternance avec les lacunes, de sorte que nous avons un grand faisceau avec groupe mécanique sous-épidermique, puis une lacune, puis de nouveau un faisceau plus petit, situé au milieu de la feuille et dépourvu de groupe mécanirjue (m) fig. 1.

A leur extrémité inférieure les faisceaux sont munis de revêtements fibreux richement développés.

Lacunes quadrangulaires enclavées dans le tissu parenchymateux, lequel présente dans ses parois quelques bifurcations de membranes. Cellules tannifères absentes.

Stomates de structure normale, dans un épiderme à parois ondulées (répondant au type représenté fig. $39 a b$ ), superficiels, exclusivement sur la page inférieure. Le lumen y est étroit, le bec bien accentué et la chambre respiratoire petite (fig. 3).

Tige creuse à arêtes hérissées de crochets, vallonnée, 
les revêtements de sclérenchyme faisant saillie en dehors.

Faisceaux en nombre considérable sur un rang, munis de gaines fibreuses larges et épaisses sur le côté qui regarde l'épiderme el présentant une interruption de chaque côté à la hauteur des gros vaisseaux.

Gaines fasciculaires composées d'éléments également forts. Epiderme zigzagué, formè de cellules à parois externes, lisses et fortes, portant des stomates superficiels analogues à ceux de la feuille.

Rhizome noueux, épais, à faisceaux concentriques, dont les phloëmes sont composés de cellules sensiblement égales.

Endoderme de force moyenne à cellules épaissies en U sur le côté interne et fortement ponctuées. Faisceaux entourés aussi d'une forte ceinture d'élèments mécaniques.

Tissu parenchymateux formé de cellules à parois minces au voisinage de l'endoderme, mais qui vont s'épaississant et se ponctuant en s'approchant de l'épiderme, où elles forment un anneau de sclérenchyme coupé ici et là par des groupes de cellules à parois plus épaisses encore et colorées en jaune.

Racine pourvue d'un péricambium entre les vaisseaux primaires et l'undoderme, lequel est formé de cellules très . allongées, comprimées dans le sens du rayon, parfois disposées sur une double rangée, à grands lumens, à lamelles moyennes fortement lignifiées et à épaississements en $U$ très prononcés sur le côté interne, tandis que les parois latérales et externes sont restées minces.

Les cellules de l'écorce confinant à l'endoderme sont pourvues d'épaisses parois et disposées régulièrement comme dans les autres espèces Nodosités en grand nombre s'avançant bien avant ver's l'endoderme.

Aux endroits d'insertion des radicelles sur la racine- 
mère, on peut observer qu'il ne se forme pas de nodosités et que, bien au contraire, les cloisons au lieu de s'amincir persistent dans la forme massive qu'elles revètent dans les couches voisines de l'endoderme et cela tout du long et tout autour de la jeune radicelle.

Cette remarque s'applique aussi bien à toutes les espèces qu'à celle qui nous occupe en ce moment.

\section{Carex Murieata. L}

Feuille portant les mèmes crochets sur ses bords libres, à épiderme lisse en coupe transver'sale, à cellules de plissement analogues à celles de C. divulsa. Mème disposition des faisceaux et des lacunes. Il en est de même des stomates et de leur's parties constituantes.

Ces stomates présentent en outre une particularité intéressante, en ce sens qu'ils renferment des gouttelettes réfringentes souvent très grosses, colorables en jaunebrun par l'acide osinique et en l'ose par la teinture l'Alkanna; offrant ainsi tous les caraclères d'un corps huileux. Ce phénomène est commun aux deux espèces, mais d'une façon plus marquée chez C. muricata.

On n'y voit pas non plus de cellules secrétant du tannin; quelques bifurcations dans les parois des cellules du parenchyme (fig. 10).

Tige de forme triangulaire pourvue de papilles en crochets sur les arêtes et présentant les mêmes vallonnements, Même analogie pour les stomates qui sont aussi superficiels.

Peut-être pourrait-on cependant constater dans les gaines fibreuses des faisceaux, une tendance à une plus grande épaisseur, surtout à l'extrémité qui regarde le cenire de la tige.

Si nous comparons les fig. 5 el 6 qui donnent une section transversale du rhizome des deux espèces, nous constaterons ici aussi une grande ressemblance. 
Les deux tiges souterraines sont également noueuses et épaisses, présentant sous l'épiderme des groupes de cellules sclérenchymateuses.

Le lissu cortical chez C. muricata, ne présente pas plus de différence. Mèmes cellules endorlerniques avec épaississement en U, mèmes éléments à parois fortes et ponctuées sous l'épiderme, fournis par le lissu extérieur de l'écorce. Même faisceilux concentriques pourvus de phloëmes analogues.

Racine assez semblable à celle de l'espèce précédente, à part quelques pelites différences sins importance résultant probablement des conditions d'habitat ou de causes accidentelles, et du reste fort inconstantes dans leur façon de se présenter.

Péricambium présent entre les vaisseaux primaires et les éléments de l'endoderme, lesquels offrent la même forme et les mêmes épaississements sur leur còté interne.

Sous l'épiderme, groupes semblables de cellules sclérenchymateuses.

En comparant entre elles, à divers âges, plusieurs racines de ces deux espèces, on trouve en général chez C. Divulsa une tendance plus marquée à l'èpaississement des cloisons cellulaires, surtout aux places d'insertion des radicelles et dans le parenchyme de l'écorce extérieure.

Dans toutes les racines jeunes - et c'est aussi, à ce qu'il nous semble, le cas pour toutes ces espèces qui présentent à l'état adulte la disposition radiale - on n'olsserve qu'une sorte de cellules dans le tissu cortical, cellules qui sont disposées en séries radiales et dont certrines d'entre elles s'allongent et collapsent en se pourroyant de nodosités, ou bien se résorbent de façon à disparaître entièrement. 
Bien qu'il soit difficile, vu la grande variété d'aspect sous laquelle s'offrent les diverses formes des espèces voisines de C. muricata, de trouver un échantillon qui réponde d'une façon certaine aux caractères extérieurs distinctifs pour $\mathrm{C}$. virens, nous donnerons cependant dans les quelques lignes qui suivent, une description brève de la structure anatomique des feuilles, tiges et racines d'une plante qui, cultivée sous le nom de $C$. virens. Lam., a montré en effet tous les caractères de cette variété, c'est-à-dire des écailles d'un vert pàle, des utricules étalés, divergents et des épillets munis d'une branche sétacée plus ou moins longue.

Nous verrons, cette étude terminée, qu'il existe, pour cette forme aussi, au point de vue anatomique une grande ressemblance, soit avec C. muricata, soit encore avec C. Divulsa.

\section{Virens Lam.}

L'épiderme de la feuille esi ici quelque peu différent de celui que l'on rencontre chez $\mathrm{C}$. divulsa. On y trouve en effet, quelques cellules allongées en papilles de forme pyramidale. Les cellules de plissement forment un groupe de six à sept élėments nettement différenciés par leur grandeur et la forme d'éventail que leur pression réciproque a donnée à leur groupement.

Pour les faisceaux, nous trouvons au lieu de groupes sclérenchymateux sous-épidermiques, séparés de l'extrémité supélieure du faisreau par un assemblage de cellules aqueuses, un revêtement fibreux, large, non interrompu, allant du faisceau à l'épiderme sans interposition d'autres éléments.

Pour ce qui est des stomates, des cellules du parenchyme et des éléments des faisceaux, nous avons correspondance entre les diverses formes.

Tige présentant des faisceaux disposés sur un rang, 
pourvus de gaines fibreuses épaisses, alternant avec des lacunes petites à contours mal définis. Du reste, grande analogie arec les formes voisines.

Pour le rhizome, la ressemblance est encore très grande, soit en ce qui concerne les faisceaux qui sonit concentriques, soil au point de vue des éléments de la gaaine qui ont mème forme et mème disposition.

La racine présente plus d'affinité avec celle de C. muricala. Chez cette dernière espèce et dans celle qui nous occupe, l'on peut constater un épaississement plus marqué des parois latérales dans les cellules endorlermiques.

Si nous résumons maintenant ce que nous avons vu dans ces trois formes, nous n'arrivons pas à trouver un caractère anatomique par lequel on puisse les distinguer facilement. De plus la grande rariété d'ètals transitoires qui existent d'une part el le peu de caractẻres distinctifs que fournit l'inllorescence d'autre part, sont autant de bonnes raisons pour que l'on regarde les deux formes divulsa et virens, comme étant des variétés du type C. muricata.

Carex vulpina L (Reich. Icon. vir, 564.)

Feuille. - Epiderme absolument lisse (sauf sur les arètes où (fig. 11) l'on trouve les mèmes crochets que chez les espèces précédentes), formé ?de cellules arrondies, plus grandes en face des faisceaux, là où il n'y a pas de revètement de sclérenchyme.

Cellules de plissement brusquement différenciées au nombre d'une douzaine, distribuées sur un seul r'ang. Stomates à niveau de l'ćpiderme.

Faisceaux rapprochés de l'épiderme inférieur, entourés de cellules aqueuses qui leur font gaine sur tout le pourtour, saul à l'extrémité inférieure.

Revètement fibreux en groupes et non en colonnes, interrompus par du parenchyme. Gaines fasciculaires 
dunt les cellules sont pourvues de grands lumens et de parois peu épaissies. A la pointe supérieure du faisceau le revêtement fibreux n'est formé que de quelques rares éléments, lesquels n'apparaissent pas du tout dans les jeunes faisceaux.

Parenchyme dépourvu de cellules secrétrices du tannin.

Cônes siliceux sur les deux faces de la feuille; stomates superficiels répondant au type ordinaire.

Tige à épiderme lisse, munie de crochets sur les arêtes dans les parties supérieures, triangulaire, à angles très aigus formant trois ailes allongées, munie de faisceaux marginaux sur un seul rang, ceux situés à l'extrémité des ailes plus grands et pourvus de revêtements fibreux plus puissants.

Centre de la tige creux, mais dont la lacune ne se continue pas dans les ailes.

Faisceaux munis de gaines fibreuses dont les éléments se répartissent le plus souvent sur trois couches à l'extrémité interne; assez nombreux, se bifurquant dans leur course et s'anastomosant entre eux par leur gaine dans le haut de la tige.

Rhizome à faisceaux concentriques répartis sur le centre du cylindre central aussi bien qu'à sa pćriphérie et dont la partie libérienne est formée de cellules de diamètre sensiblement égal.

Entourés par une gaine de cellules fibreuses dont les cloisons sont considérablement épaissies, ils sont séparés les uns des autres par une mince lame de parenchyme. Vers la périphérie du cylindre central, ils se rapprochent tellement les uns des autres que leurs gaines s'anastomosent.

Endoderme fortement épaissi sur le côté interne.

Parenchyme cortical riche en méats intercellulaires, dont les cloisons vont s'épaississant peu à peu en avan- 
çant vers l'épiderme sous lequel elles forment un tissu sclérenchymateux, coupé çà et là par des petits groupes arrondis d'éléments fibreux plus épaissis. Cet anneau de sclérenchyme est richement pourvu de ponctuations et de méats intercellulaires. Vues de face, ces ponctuations présentent l'aspect de grands cercles entourant un ou deux autres cercles plus petits, plus ou moins réguliers de forme (fig. 12).

Racine dont les vaisseaux primaires confinent directement à l'endoderme, sans interposition de péricambium et dont les lames vasculaires se trouvent interrompues dans leur disposition radiale.

Endoderme dont les éléments prennent une épaisseur considérable sur le côté interne, ont une forme (fig. 13) régulière et s'appuient sur une zone péricambiale lignifiée interrompue. Dans les vieilles racines, le parenchyme cortical se résorbe, mais il reste toujour's trois ou quatre rangées de cellules disposées radialement, à parois massives, à contours ovales, laissant entre elles des méats prononcés, de sorte que l'endoderme se trouve à son tour recouvert d'un anneau protecteur.

A la périphérie, anneau sclérenchymateux formé de plusieurs rangs de cellules polygonales.

Carex paniculata L. Reich. Icon. vill, 574).

Feuille épaisșe à épiderme supérieur lisse, formé de cellules à lumens arrondis, régulières ; sur la page inférieure, cellules beaucoup plus petites, développées en papilles nombreuses et en stomates.

Cellules de plissement très peu différenciées, au nombre de cinq ou six seulement, sur un seul rang, confinant directement aux fibres de la nervure médiane, bordées sur les côtés d'une rangée d'autres cellules plus petites et incolores et dont les points de jointure avec le premier rang prennent l'aspect collenchymateux. 
Faisceaux rapprochés de l'épiderme inférieur n'occupant jamais avec leur's revêtements toute l'épaisseur de la feuille, - a part toutefois celui de la nervure médiane - séparés par des lacunes grandes, allongées dans le sens de la leuille el pourvus d'un système de cellules aqueuses très développé.

En général, on n'a pas dans ces feuilles de colonnes de sclérenchyme continues, mais seulement des groupes de cellules scléreuses sous l'épiderme en regard des faisceanx. On y rencontre à la place d'un stéréome en colonnes, traversant l'épaisseur, des bandes larges de cellules aq̣ueuses.

Gaines fasciculaires formées d'éléments épaissis surtout à la hauteur de la séparation des deux parties du faisceau.

Les parois de cellules chlorophylliennes présentent dans leur milieu un grand nombre de méats (bifurcations). Bords de la feuille pourvus de cellules développées en papilles sailiantes.

Stomates enfoncès sous l'épiderme de la page inféricure et dont les cellules de ferneture se trouvent en partie masquées par des épaississements frangés, irréguliers, produits par les parois des cellules annexes (fig. $30 a$ et $30 b$ ).

Cette espèce sera donc très facilement reconnaissable, gràce à la structure spéciale de son appareil slomatique.

Tige triangulaire (fig. 15), dont les angles portent une cellulo transformée en papille plus ou moins aiguë et présentant ici et là sur les côtés une on plusieurs cellules faisant aussi saillie an-dessus des autres ćléments de l'épiderme.

Faisceaux disposés sur un rang dont les parties présentent la même structure que dans la feuille.

Cellules de l'épiderme pourvues de parois extérieures épaisses avec slomates semblables de forme et de disposition à ceux de la feuille. 
Le parenchyme vert offre aussi une grande quantité de méats intercellulaires dont l'ensemble a pour résultat un tissu spongieux.

Rhizome de structure ordinaire offrant un anneau sclé. renchymateux formé d'éléments massifs qui se continuent par un parenchyme à méats.

Endoderme épaissi en U. Faisceaux concentriques à phloëmes normaux entourés de gaines fibreuses. L'écorce est dépourvue de lacunes aérifères.

Racine à endoderme fort, régulier, à éléments épaissis en $\mathrm{U}$, à zone péricambiale souvent interrompue par les vaisseaux primaires.

Tissu cortical distribué d'après le type C. maxima, en toile d'araignée avec nodosités très prononcées (fig. 16) et dont la plupart paraissent formées par des plissements de la membrane en même temps que par collapsus.

Carex Davalliana L.

(Reich. Icon VIII, 523. C. dioïca. Host.)

Feuille de forme toute spéciale (fig. 45) sans autre nervure apparente que la nervure médiane.

Epiderine fort sur la face supérieure et dont les cellules arrondies ont la paroi externe plus épaisse que les autres.

A la coupe transversale, cette feuille présente la forme d'un triangle dont les trois angles sont occupés par un groupe d'éléments mécaniques $(m)$. Cônes siliceux à la face infërieure dans les cellules de l'épiderme qui font visà-vis aux groupes d'éléments mécaniques ci-dessus mentionnés.

Cellules de plissement absentes à moins que l'on ne considère comme telles toutes les cellules de l'épiderme sur la page supérieure; en tous cas très peu différenciées.

Faisceaux occupant le centre de la feuille et non les bords, à gaine fasciculaire forte, à revêtcments fibreux 
développés surtout à l'extrémité qui r'egarde la page inféricure. Ils sont entourés d'une gaine parenchymateuse (cellules aqueuses) interrompue aux deux bouts des grands faiscedux, mais continue autour des petits.

Lacunes très grandes, souvent eneore remplies des débris le cellules résorbées; deux d'entre elles, plus grosses que les autres, occupent le eentre de la feuille.

Stomates superficiels, répondant comme structure au type de la fig. 39 b. munis de becs prononcés à l'entrée de l'ouverture stomatique, à lumens étroits el allongés résultant d'un épaississement considérable des parois. Exclusivement situés sur la page inférieure de la feuille, ils présentent, vus de face, une figure analogue à fig. 396 .

Tige triangulaire, erénelée, à épiderme formé de cellules ici et là développées en papilles (fig. 42) en forme de boucles en coupe transversale et de eroehets en coupe longitudinale et pourvue de stomates superficiels semblables à ceúx de la feuille. Couches cuticulaires très netles. Faisceaux sur un rang, alternant régulièrement entre petits et grands, et disposés de façon qu'à chaque angle se trouve un grand faisecau et entre ceux-ci trois autres de même grandeur au milieu des côtés.

Entre ces divers faiseeaux viennent s'intercaler des lacunes arrondies présentant un diamètre senšiblement égal. Gaines faseiculaires dont les éléments présentent un épaississement maximum sur le pourtour du liber, et sur les flancs du faisceau en regard des cellules aqueuses.

Tige souterraine dontle tissu parenelyymateux se résorbe de fort bonne heure. On peut eependant, dans des rhizomes plus jeunes, constater la présence de lacunes qui sont les premiers commencements de la résorption.

Cellules endodermiques épaissies en $U$, fortement accentuées sur leurs parois internes.

Faisceaux concentriques à phloèmes eomposés de eel- 
lules à peu près égales. Structure habituelle des rhizomes à faisceaux concentriques, ne présentant pas de particularité r'emarquable.

Racine grêle sans assise péricambiale entre les vaisseaux primaires el les cellules de l'endoderme. Ces dernières très fortes avec épaississement en $U$ donnạt au lumen la forme d'un ètrier (fig. 46.)

Cellules du parenchyme cortical disposées en séries radiales, allant croissant en diamètre ver's la périphérie. Sur les parois des cellules étirées tangentiellement, nodosités nombreuses très prononcées (fig. 47). .

L'espèce voisine, Carex Dioïca L. ${ }^{1}$ présente une grande analogie au point de vue de la structure anatomique. Par contre, les caractères extérieur's des feuilles, du rhizome, des épillets el des utricules suffisent déjà pour le différencier du précédent.

Feuille semblable de forme en coupe transversale, présentant le même arrangement dans ses élćments.

Comme chez C. Davalliana, nous avons aux angles des groupes de cellules mécaniques. Les groupes correspondant aux arêtes de la feuille n'ont aucune relation avec les faisceaux libéro-ligneux; celui de l'épiderme inférieur forme au contraire un revètement au faisceau de la nervure médiane.

Sur le còté opposé à celte nervure se trouvent aussi des cellules épidermiques allant en augmentant de grandeur vers le centre et que l'on peut regarder comme étant l'équivalent des cellules de plissement. Disposition analogue des lacunes.

Tige très arrondie, par endroits presque ronde el conservant cette forme jusque dans les parties supérieures.

Epiderme de mème force que chez la précédente espèce,

${ }^{1}$ Reich. Icon. VIII, 522. 


\section{6}

mais dépourvu de papilles ou à peu près complètement. Mêmes zones culiculaires bien marquées, mêmes stomates superficiels.

Nême arrangement des faisceaux libéro-ligneux par rapport aux lacunes.

Le rhizome est ici différent; tandis que celui-ci chez Davalliana est cespiteux, il est, dans l'espèce qui nous occupe, stolonifère, et en même temps aussi cespiteux ; à chacune de ces formes correspondent des faisceaux libéroligneux différents : en effet, dans les stolons, nous trouvons exclusivement la structure collatérale, tandis que dans la partie vraiment souterraine du rhizome (cespiteuse), nous avons des faisceaux concentriques.

Les collatéraux sont serrés sur un rang contre les cellules endodermiques et présentent ici et là une forme intermédiaire.

Outre l'endoderme, dont les éléments sont très fortement épaissis en forme de croissant et parfois sur un double rang, les faisceaux du cylindre central sont encore protégés par des gaines lasciculaires formées de cellules massives qui s'anastomosent entre elles.

Le parenchyme cortical se résorbe de bonne heure, en formant de grandes lacunes séparées les unes des autres par des colonnes de cellules rondes, qui rayonnent du. eylindre central et vont rejoindre l'anneau sclérenchymateux à la périphéric.

La racine a une grande ressemblance avec celle de C. Davalliana.

Sa structure est celle que l'on trouve communément cher les Carex avec tissus mécaniques assez richement développés : en effet, nous trouvons à la périphérie un anneau de sclérenchyme composé d'éléments massils, un parenchyme rayonné abondamment pourvu de nodosités, et un endoderme à cellules très fortement épaissies. 
La zone de péricambium se trouve ici très souvent interrompue par les vaisseaux primaircs.

Comparées entre elles au point de vue de leur structure anitomique, ces deux espéces sont loin d’ètre identiques, mais elles n'en montrent pas moins de grandes resseniblances qui sont dues probablement à des conditions de végétation semblables dans des marais tourbeux.

Carex chordorrhiza. Ehrh (Reich. Icon., VIII, 535).

Feuille petite, linéaire, ramassée sur elle-même, pourvue de rleux épidermes d'inégale force, le supérieur hérissé de papilles surtout en regard des faisceaux, l'inférieur lisse, tous deux richement pourrus de cônes siliceux.

Lacunes quadrangulaires au nombre de trois ou quatre seulement, alternant arec des faisceaux qui occupent presque ìe centre de la feuille, sont munis à leur's deux extrémités de revètements fibreux formant colonnes, et pourvus sur leurs flancs de cellules aqueuses.

C'cllules de plissement grandes, comprimées entre elles dans le sens du rayon, à parois mitoyennes minces.

Stomates situés exclusivement sur la page inféricure, superficiels et correspondant dans leur structure au type ordinaire.

Tige triangulaire à angles très arrondis, franchement creuse dans sa partie inférieure, à épiderme lisse et parenchyme ne renfermant qu'un rang de faisceaux marginaux pourvus de gaines fasciculaires fortes et de revêtements fibreux assez puissants. Parois externes de l'épiderme très fortes, soutenues souvent par des cônes siliceux.

Au voisinage des épillets, les angles de la lige s'accentuent, ce qui donne unc forme plus neltement triangulaire.

Rhizome de structure spéciale, présentant deux aspects différents, suivant que l'on en considère la partie traçante ou la région souterraine. 
Lans le premier cas, nous n'avons pas de cylindre central, mais a sal place une grande lacune.

Le tissu qui limite le centre creux est du parenchyme qui renferme des faisceaux nettement collatéraux formant une rangée régulière. Outre la lacune centrale, nous avons encore à la périphérie des lacunes plus petites, séparées les unes des autres par des rayons de parenchyme.

Ces rayons portent, eux aussi, des faisceaux collatéraux. Sous l'épiderme, on observe une forte cóuche de cellules mécaniques formant un anneau continu.

Examinés à un plus fort grossissement, les faisceaux, tant ceux de la rangée interne que ceux des rayons, sont entourés d'une gaine de fibres, plus épaisse sur le côté intérieur ; leur gaine fasciculaire est elle-même continue et formée d'éléments s'épaississant à la hauteur de la ligne de sëparation des deux parlies du faisceau. Leurs phloëmes présentent ici une structure très normale.

Tout autre est la structure des parties sonterraines qui, au lieu de présenter des faisceaux collatéraux, offrent au contraire exclusivement la forme concentrique et la distribution ordinaire générale des rhizomes cespiteux. Les choses ont donc lieu ici comıne chez C. diö̈ca, examiné plus haut.

Parenchyme riche en méats de toutes sortes.

Quant à l'anneau de sclérenchyme, on y distingue deux parties, l'une plus interne formée de deux, trois et quatre couches de cellules à parois épaisses et lignifiées; l'autre, sous-épidermique, rappelant par la forme de ses éléments ceux de l'endoderme du cor'ps central dans d'autres espèces (fig. 48) s.

L'épiderme est ici aussi constitué d'une façon particulière. En coupe transversale, ses éléments se présentent très épaissis, avec petits lumens refoulés ver's l'anneau sclérenchymateux et munis de replis en tous sens prove- 
nant des ondulations des cloisons; les stries des couches cuticulaires s'y laissent facilement apercevoir.

$\mathrm{Vu}$ de face, cet épiderme présente des parois fortement sinueuses, épaisses, s'engrenant les unes dans les autres très profondément; en coupe longitudinale radiale, ces cellules offrent un grand épaississement des parois externes et les lumens très réduits. Elles sont en général colorées en jaune-brun (fig. 49 a. b.)

Racine présentant l'aspect ordinaire en toile d'araignée, à épiderme quelque peu poilu, soutenu par un fort anneau de sclérenchyme.

Cylindre central protégé par un endoderme solide dont les éléments se sont considérablement épaissis (fig. 50) sur leur face interne et présentent des lumens pyriformes.

Lames vasculaires se prolongeant assez loin dans le parenchyme central.

Vaisseaux primaires confinant directement à l'endoderme sans interposition de cellules péricambiales.

La dernière couche de l'anneau sclérenchymateux (fig. 51) est formée de cellules semblables à celles qui font fonction d'épiderme, seulement par épaississement et lignification de leurs parois, elles ont pris l'aspect de cellules endodermiques $(s)$.

Carex Schreberi. Schrank. (Reich. Icon. VIIl, 540).

(C. præcox. Schreb. non Jacq.)

Feuilles à épidermes hérissés de papilles (fig. 52) de formes pyramidales très aiguës; à la page inférieure ces papilles offrent des contours plus arrondis et moins saillants que sur la page supérieure où les cellules sont d'un diamètre quelque peu plus grand. De plus, on observe qu'elles se rencontrent de préférence sur les feuilles les plus élevées sur la tige; pendant que celles qui occupent les parties inférieures de la plante paraissent n'en posséder que fort peu. 


\section{0}

Cellules de plissement sur un rang nettement différenciées et allant par gradation de grandeur.

Cônes siliceux dans les deux épidermes, rares sur la page supérieure où ils se trouvent localisés aux extrémités de la feuille, à l'endroit où les cellules épidermiques diminuent de diamètre, mais augmentent en épaisseur dans leurs parois.

Faisceaux plutôt marginaux, pourvus de gaines fasciculaires à larges éléments épaissis sur le côté interne et entourées d'une bande de cellules aqueuses.

Lacunes centrales enveloppées de plusieurs couches de tissu chlorophyllien, tont les cellules laissent voir de nombreux méats soit en forme d'entonnoir /Trichterzellen), soit de forme habituelle.

Pas de cellules secrétant du tannin.

Stomates analogues à ceux de C. Oederi (fig. 18) munis de grandes chambres respiratoires et de cellules annexes plus petites que les autres éléments épidermiques.

Tige ronde, creuse au centre, à épiderme (fig. 53) formé de cellules ovales, à parois externes épaisses et dont les lumens sont peu ou point aplatis tangentiellement. Ici et là, l'une d'entre elles proémine sur ses voisines.

Cônes siliceux dans les éléments épidermiques qui font vis-à-vis aux revêtements sclérenchymateux.

Lacunes arrondies en alternance avec les faisceaux, lesquels sont pour la plupart d'assez forte taille, et possèdent des gaines ponctuées dont les parois cellulaires s'épaississent à la hauteur de la ligne de séparation entre le phloème et le xylème, et surtout autour des tubes criblés.

Tout le faisceau est lui-même entouré par une gaine fibreuse formant plusieurs couches, mais coupée par le tissu vert et les cellules aqueuses sur les côtés.

Stomates comme dans la feuille, superficiels, mais quelque peu plus grands. 
Tout au haut de la tige, les faisceaux quittent pour la plupart le bord sous l'épiderme et se rapprochent du centre, en même temps que le contour de l'organe prend une forme nettement triangulaire.

Rhizome traçant dont le parenchyne cortical est pourvu de lacunes séparées les unes des autres par des colonnes de parenchyme à cloisons minces.

A l'extérieur sous l'épiderme, se trouvent plusieurs couches de cellules à parois plus épaisses, quoique encore relativement minces et dont les éléments se cloisonnent tangentiellement (fig. 54.)

L'épiderme est lui-même formé d'éléments semblables et aplatis tangentiellement, mais de volume plus petit (fig. 54 ép.)

Faisceaux répandus sur toute la surface du corps cenıral, concentriques, à phloèmes normaux, entourés de gaines fibreuses souvent anastomosées entre elles. A la périphérie du cylindre central s'observent quelques formes de passage all type collatéral, sans qu'il soit cependant possible de le trouver nettement constitué.

Cellules endodermiques à épaississements internes peu saillants, tantôt arrondies, ovales ou triangulaires suivant leur position (fig. 55.)

Les éléments situés sous l'épiderme, riches en ponctua- . tions surtout visibles sur des coupes longitudinales, renferment de petites gouttelettes d'une matière oléagineuse, colorable en noir par l'acide osmique, indifférente à l'action du bichromate de potasse, el qui communique au rhizome une odeur particulière. Ces gouttelettes sont solubles dans un mélange d'alcool et d'éther à chaud (fig. 540 .)

Racine dont l'épiderme est doublé d'un anneau solide de cellules mécaniques réparties sur cinq ou six couches.

Endoderme à bord interne peu accentué, formé d'éléments d'épaisseur sensiblement égale sur tout leur pourtour 
et dont la forme des lumens est celle d'un ovale allongé (fig. 56.)

Il cxiste entre cet endoderme et les vaisseaux primaires un péricambium continu, régulièrement développé.

Sur le côtć extérieur de la gaine, les éléments du parenchyme cortical interne revêtent des parois épaisses, laissant entrc elles des méats; cet épaississement ne s'étend guère au-delà des deux premières assises, pour faire place ensuite à d'autres assises en voie de collapsus et de résorption.

Carex Brizoödes. L. (Reich. Icon. VIII, 548).

Epiderme plus ou moins lisse sur les deux faces présentant en coupe transversale quelques protubérances papilloïdes (surtout vers l'extrémité de la feuille), plus nombreuses chez les feuilles qui se trouvent sur la tige (fig. $58 a$ ). mais toujours en moins grande quantité que dans l'espèce précédente.

Sur la page supérieure, les cellules sont environ le double plus grandes que sur la page inférieure. En regard de la nervure médiane, elles se transforment brusquement en cellules de plissement, grandes, à parois minces, non collenchymateuses, disposćcs sur un seul rang au nombre de quatre ou cinq environ. Tout le contour de la coupe est plus ou moins vallonné par endroits.

Faisceaux rapprochés de l'épiderme inférieur à gaine fasciculaire épaissie à la hauteur de la ligne de séparation du bois et du liber; tout le faisceau est lui-mème entouré de fibres formant une enveloppe protectrice interrompue sur les côtés par des cellules aqueuses.

Lacunes arrondies, centrales, entourées de plusieurs couches de cellules vertes.

Stomates sur la face inférieure, superficiels, à petites chambres. construits comme dans l'espèce précédente.

Én coupe longitudinale, les feuilles présentent un tissu 
spongieux et des cellules épidermiques qui, sur la lace supérieure, portent chacune une papille en forme de crochet généralement rabaltu sur la surface de la feuille. A la face inférieure, les cellules épidermiques laissent voir un nombre considérable de cônes siliceux qui apparaissent d'une façon très nette sur les préparations faites à la fuchsine ammoniacale. Ces cônes se trourent quelquefois en grand nombre dans une mème cellule (fig. $58 \mathrm{~b}$.)

Tige polygonale dans le haut, où chaque angle est orcupé par un gros faisceau entourè d'une gaine semblable à celles des faisceaux foliaires et où l'épaississement se continue tout autour du phloème.

A la pointe externe de ces gros faisceaux s'attache un revètement de cellules fibreuses qui s'étend jusqu'à l'épiderme, lequel renferme aussi des cellules à cônes siliceux. L'autre pointe du faisceau est protégée par une double assise de ces mèmes éléments.

Entre chacun des gros faisceaux, au milieu des côtés du polygone, s'en trouve un plus petit - quelquefois deux séparé de l'épiderme par du tissu chlorophyllien.

Stomates complètement superficiels à lumens quelque peu plus grands que dans l'espèce précédente.

En coupe longitudinale, tout l'épiderme paraît être richement pourvu de crochets-papilles (fig. 59.)

Rhizome traçant, $\dot{a}$ faisceaux concentriques, dont les phloèmes présentent une constitution normale (tubes criblés à diamètre plus grand et cellules annexes plus petites) et dont les gaines fibreuses s'anastomosent souvent entre elles.

Nême parenchyme lacuneux et même sclérenchyne que dans l'espèce précédente.

Les cellules de l'endoderme présentent à elles seules un aspect un peu différent, leur côté interne étant plus fortement constitué (fig. 57.) 


\section{4}

Celte tendance à une plus grande résistance dans les tissus se retrouve aussi dans les gaines fasciculaires du cylindre central et constitue la différence la plus frappante entre les deux espèces.

Ponctuations nombreuses, visibles surtout en coupe longitudinale, dans les cellules à la périphérie, et mêmes matières huileuses que dans l'espèce ci-dessus.

Dans la racine, nous trouvons aussi une structure plus forte, soit dans les cellules endodermiques, soit dans le parenchyme du cylindre central.

La forme des éléments de l'endoderme est chez C. brizoïdes moins ovale ; par contre leur épaissement en $U$ y est plus considérable. lls renferment de plus une assez grande quantité d'amidon.

Quant aux vaisseaux primaires, le péricambium étant ici lignifié (comme dans l'espèce précédente), d'une façon complète, il devient parfois difficile de distinguer comment ils se comportent vis-à-vis de l'assise péricambiale.

Carex Ieporima H. (Reich. Icon. VIII, 554).

C. ovalis. Good

Feuilles à épiderme lisse dont les cellules sur la face supérieure sont plusieurs fois plus grandes que celles de la face inférieure (fig. 61).

Cellules de plissement grandes, graduellement disposées, comprimées radialement et sur un seul rang.

Lacunes allongées dans le plan parallèle aux deux épidermes. Cellules à cônes siliceux sur les deux faces.

Faisceaux disposés au bord de la feuille, munis à leurs extrémités de revêtements fibreux interrompus sur les flancs, à gaines fasciculaires fortes. Cellules à chlorophylle disposées en couches plus nombreuses sur le côté inférieur des lacunes, réduites le plus souvent à ne former qu'une ou deux assises entre l'épiderme supérieur el la lacune.

Stomates petits, superficiels, exclusivement sur la face 


\section{5}

inférieure de la feuille, enclavés dans un épiderne zigzagué.

Tige polygonale, creuse au centre, dont l'épiderme est formé de cellules à parois externes considérablement épaissies et portant des cônes siliceux pelits en regard des revêtements fibreux. Lacunes à peu près nulles dans le haut de la tige, toujours petites dans le bas.

A chaque angle du polygone, la cellule terminale est agrandie et prend en général la forme d'un triangle dont les parois externes s'épaississent fortement.

Aux arêtes correspondent des faisceaux libéro-lị̣neux plus gros que les autres; entre ceux-ci, au milieu des côtés, se trouve un autre faisceau d'égale taille environ ; l'espace restant est occupé par d'autres plus petits, de sorte (ju'on a successivement alternance de grands et de petits faisceaux. Tous sont pourvus de gaines dont les éléments sont épaissis sur leur côté interne. Outre cette première enveloppe, ils sont protégés par des revêtements fibreux, qui, sur le côté correspondant au xylème s'anastomosent quelquelois, emprisonnant ainsi dans leur ceinture le tissu chlorophyllien et le séparant du parenchyme médullaire (fig. 62.)

Quant aux stomates, ils présentent la même structure que dans la feuille; ils sont superficiels et de même grandeur, mais on observe ici que la paroi épaissie de l'épiderme s'incurve quelque peu des deux côtés du stomate vers l'ouverture.

Vues en coupe longitudinale, les cellules épidermiques, outre leur grande épaisseur sur le côté extérieur, se montrent allongées, pourvues de lumens (fig. 63) étroits et comprimés. De face, nous avons comme à l'ordinaire des parois zigzaguées, plus épaisses que celles de la feuille.

Rhizome semblable à celui de $\mathrm{C}$. punctata quant à la distribution des tissus dans le parenchyme cortical. 
11 en est de même des éléments de l'endoderme, lesquels sont plus ou moins comprimés et s'appuient par leur face interne sur une couche de cellules péricambiales à parois épaisses.

Faisceaux concentriques entourés d'une forte gaine fasciculaire à phloèmes normalement constitués.

Parenchyme cortical riche en méats intercellulaires.

Racine où le péricambium n'existe seulement qu'en quelques endroits entre les vaisseaux primaires et l'endoderme.

Dans ce dernier, les éléments offrent à l'œil des contours plus ou moins arrondis et des épaississements relativement peu prononcés et peu ponctués, presque égaux comme force aux premières cellules du. tissu (fig. 64) cortical interne, vers le cylindre central. Le reste du tissu, situé sous l'anneau sclérenchymateux, ne tarde pas à tomber en collapsus jusqu'à l'avant-dernière assise du côté de l'endoderme.

Même disposition que chez C. punctata, quant à l'arrangement en séries radiales et aux nodosités.

Carex Buxbaumii. Wahlg. (Reich. Icon. VIII, 589.)

Fenille. - Epidermes des deux faces de force à peu près égale, tous deux hérissés de papilles, allant souvent par paires, c'est-à-dire formées par deux cellules voisines se développant chacune en deux protubérances pointues (fig. 65).

Cellules de plissement plus ou moins brusquement différenciées et dont les parois radiales acquièrent une épaisseur relativement considérable.

Faisceaux rapprochés de l'épiderme inférieur, munis de cellules aqueuses sur les còtés et le long des colonnes du stéréôme.

Gaines fasciculaires composées de cellules fortes et cela sur tout le pourtour du faisceau libéro-ligneux. 
Lacunes allongées perpendiculairement à la nervure médiane et entourées de cellules tannifères répandues sans ordre régulier dans les tissus.

Stomates superficiels, sur les deux faces de la feuille et dont les cellules annexes se prolongent en haut et en bas par dessus les extrémités des cellules stomatiques (fig. 32).

Vus de face, ces organes se présentent entourés de cellules à contours zigzzagués et munies à leur intérieur d'un anneau plus ou moins rond qui représente la papille; les cellules anmexes du stomate forment ici (comme chez C. stricta) deux renfoncements opposés en regard de l'ouverture.

Tige triangulaire à angles très aigus, présentant deux sortes de faisceaux libéro-ligneux; les uns sont marginaux, revêtus d'épaisses gaines fibreuses sous épidermiques, les autres plus intérieurs en sont dépourvus et présentent leur sclérenchyme le plus épais sur l'extrémité correspondant au bois.

Ces faiscenux, les petits et les grands, au nombre d'une trentaine environ, font alternance entre eux et avec les lacunes.

A chaque angle, l'épiderme de la tige se trouve renforcé par un groupe d'éléments sclérenchymateux.

Gaines fasciculaires pourvues d'èléments dont l'épaississement maximum s'observe autour du liher. Cellules aqueuses formant autour du faisceau une seconde gaine parenchymateuse incolore.

Epiderme papilleux, pourvu de cônes siliceux el de stomates superficiels, analogues à ceux de la feuille.

Le parenchyme chlorophyllien offre ici et là des bifurcations et se trouve réduit à quelques rangées de cellules sous-épidermiques secrétant du tannin en assez grande quantité. 
La tige ne se creuse ici ni dans le haut, ni dans le bas; dans sa partie inférieure, les nervures principales font saillie au dehors el donnent à. la coupe transversale un aspect crénelé.

Rhizome à faisceaux concentriques dont les phloèmes présentent la structure normale.

Endoderme composé de cellules épaissies tantôt en U tantôt en croissant, quelquefois double.

Gaines fibreuses formées de plusieurs couches, ne s'anastomosant qu'à la périphérie du cylindre central, donnantainsi àcelui-ci une puissance de résistance considérable.

Parenchyme cortical formé d'éléments à cloisons minces, arrangés radialement et augmentant de diamètre du centre à la périphérie.

Dans les rhizomes àgés, quelques-unes de ces séries radiales de cellules se résorbent, amenant ainsi la formation de lacunes allongées dans le même sens, et de colonnes de parenchyme, lesquelles portent de pelits faisceaux libéro-ligneux de structure collatérale.

A la périphérie, court un anneau sclérenchymateux continu.

Racine solidement bàtie, présentant un anneau épais de cellules sclérenchymateuses sous l'épiderme.

Au voisinage de l'endoderme, on n'observe pas de collapsus dans les éléments corticaux qui gardent ainsi une disposition normale parfaite en séries rayonnées de cellules, les unes rondes, les autres étirées et pourrues de nodosités.

S’il en est ainsi des cellules corticales, il en est aussi de même pour les méats intercellulaires qu'elles laissent entre elles, méats réguliers en losanges el dont le diamètre va en augmentant ver's la périphérie.

Epiderme pourvu de longs poils, en apparence cloisọnès (fig. 66). 
Endoderme composé d'éléments massifs, à lumens pyramidaux, par endroits double (fig. 67).

Lames vasculaires courtes, les gros vaisseaux étant situés très près des vaisseaux primaires, qui pour la plupart, sauf de rares exceptions, confinent directement à l'endoderme.

Parenchyme ligneux muni aussi de cloisons très épaisses.

Carex atricta, Good. (Reich. Icon. VIII, 583.)

(Carex cespitosa L. sec. Gay).

Feuille. - Epiderme supérieur à cellules arrondies, à parois extérieures lisses, dont les éléments sont d'égale grandeur. A la page inférieure, la plupart des cellules épidermiques sont développéés en papilles.

Cellules de plissement collenchymateuses, sur deux ou plusieurs rangs.

Faisceaux libéro-ligneux à gaine fasciculaire forte, bordée sur ses côtés de cellules aqueuses qui se continuent en colonnes pour aboutir à des groupes sclérenchymateux sous-épidermiques, comme chez C. paniculata.

L'aspect plus ou moins glauque que présente cette espèce parait être le résultat d'un jeu des rayons lumineux au milieu des papilles, les parois des éléments épidermiques ne paraissant pas pourvues de corps cireux déposés à leur intérieur.

Parenchyme vert parsemé de cellules secrétant du tannin.

Stomates petits, légèrement enfoncés dans le tissu, les cellules annexes entourant complètement, quand l'organe est bien développé, les cellules stomatiques.

Celles-ci présentent chacune en haut et en bas deux bandes d'épaississement qui apparaissent sous la forme d'un X (fig. $29 a, b$ ).

Examinés de face, ces stomates se présentent enfoncés sous deux cellules annexes dont les parois opposées et situées au-dessus de l'ouverture stomatique s'invaginent 
chacune pour leur compte, formant ainsi au-dessus de la chambre une ouverture en croix avec celle du stomate proprement dit (fig. 29 b).

Endoderme du cylindre central à éléments fortement épaissis dans les parties droites du rhizome.

Tige aérienne, triangulaire à angles aigus, à épiderme papilleux portant les mèmes stomates que la feuille, à angles hérissés de crochets, surtout dans ses parties supérieures.

Faisceaux libéro-ligneux nombreux, au nombre d'une cinquanlaine, sur deux rangs, entourés de gaines fibreuses épaisses, présentant des transitions à la forme concentrique à la hauteur du dernier nœud, en même temps que les gaines s'anastomosent et que les faisceaux quittent le bord de la tige.

Rhizome à épiderme zigzagué, présentant sous les cellules de celui-ci, un anneau sclérenchymateux dont la dernière assise est formée d'éléments considérablement plus forts que ceux des autres.

Parenchyme cortical offrant ici et là des lacunes rayonnant du cylindre central et provenant de la résorption d'une file radiale de cellules. A ce lissu lacuneux succèdent sur quatre couches d'éléments lignifiés et prosenchymateux les cellules de l'anneau ci-dessus mentionné.

Ponctuations, méats et bifurcations répandus en yrantité, apparaissant suivant leur position sous l'aspect de cercles disposés sans ordre au milieu des parois, ou sous celui d'une série de renflements percés à jour el ajoutés bout a bout (fig. 69).

Faisceaux libéro-ligneux arrangés pèle-mèle, concentriques ou collatératx, avec toutes les formes intermédiaires. La forme concentrique est cependant celle qui prédomine.

Racine velue dont les premières assises cellulaires 
(fjg. 94) de l'écorce extérieure revètent un caractère sclérenchymateux prononcé; dans la partie moyenne la structure radiale régulière en toile d'araignée est facilement reconnaissable.

Endoderme du cylindre central à éléments très épaissis sur leur moitié interne et. offrant une forme de croissant plus ou moins comprimé radialement. Cet endoderme s'appuie sur un péricambium très scuvent interrompu par les vaisseaux primaires.

Quant aux cellules de l'assise pilifère, elles sont pourvues de parois minces, allongées dans le sens du rayon; quelques-unes d'entre elles persistent à l'état de poils souvent très longs.

Carex vulgaris. (Reich. Icon. VIII, 580.)

Feuille très fortement construite, à épidermes de force presque égale sur les deux pages et à parois externes épaisscs.

Cellules de plissement sur plusieurs rangs, collenchymateuses, formant en regard de la nervure moyenne tout un complexus d'éléments incolores.

Sur la page inférieure, l'épiderme reste lisse; quelquefois, mais rarcment, on observe sur l'autre, un déreloppement de papilles.

Stomates en aussi grand nombre à la page supérieure qu'à la page inférieure, sinon plus grand sur la première, très légèrement enfoncés, à cellules annexes recouvrant - comme dans l'espèce précédente - le: cellules stomatiques, en laissant entre leurs parois un méat considérable, à contours heaucoup plus évasés que chez C. stricla.

Les cellules annexes renferment en outre des gouttes d'un corps huileux très réfringent, soluble tlans l'alcool à chaud et se colorant en noir par l'acide osmique et en rose par la teinture d'alkanna (fį̣. $36 a, b$ ).

Parenchyme dépourvu de cellules secrétant du tannin. 
Faisceaux entourés d'une forte gaine doublèe de cellules aqueuses, occupant tantôt toute l'ćpaisseur de la feuille, tantôt seulement (ce sont les plus petits) son bord inférieur. Lacunes grandes, plus ou moins arrondies ou quadrangulaires. Tige à structure de résistance (triangulaire), munie à chaque angle d'un groupe d'éléments sclérencliymateux s'étalant sous l'épiderme.

Faisceaux répartis sur deux rangs suivant le type ordinaire, munis de revêtements mécaniques très épais allongés en colonnes, et de gaines fasciculaires également fortes sur tout le pourtour. Ceux du rang intérieur sont aussi pourvus de gaines fibreuses forles en plusieurs couches, principalement à l'extrémité du faisceau qui regarde le centre de la tige.

Epiderme à parois externes massives, lisse, portant les mêmes stomates que la feuille.

La même structure de résistancé se retrouve dans le rhizome. Nous avons en effet à la périphérie un épiderme à fortes parois doublé d'un anneau sclérenchymateux très puissant formé de cellules massives à lumens très petits dans les deux dernières couches à l'extérieur.

Endoderme composé d'éléments épaissis en croissant, parfois double, s'appuyant'à une zone de fortes cellules péricambiales qui court, triple ou double, sans interruption entre eux et les faisceaux.

Ces derniers, de structure concentrique, sont pourvus de gaines fibreuses qui s'anastomosent à la périphérie du cylindre central.

Parenchyme cortical compose de cellules rondes, arrangées en séries plus ou moins radiales dans l'écorce interne, munies de cloisons déjà très capables de résistance et renfermant beaucoup d'amidon.

Racine velue, solidement construite présentant sous son épiderme un sclérenchyme puissant, allant en dimi- 
nuant de force vers le centre. Parenchyme cortical en toile d'araignèe. Endoderme extraordinairement inassif, composé d'éléments dont l'épaississement de la paroi interne occupe presque tout le volume de la cellule, ne laissant ainsi qu'un tout petit lumen.

Péricambium presque toujours interrompu par les vaisseaux primaires.

Carex acuta. L. [Reich Icon VIII, 584 (C. gracilis Curt)]

Feuille dont l'épiderme offre des éléments arrondis à lumens plus grands sur la face supérieure et à parois lisses. Sur la face inférieure, nous avons un riche développement de cellules en papilles (fig. $24 b$ et $70 a b$ ). Cellules de plissement disposées sur un seul rang.

Dans le parenchyme vert, les cloisons cellulaires présentent des bifurcations nombreuses.

Faisceaux libéro-ligneux occupant le bord inférieur de la feuille; les plus grands d'entre eux envahissent avec leurs revètements toute l'épaisseur de la coupe. Les cellules aqueuses les enveloppent d'une gaine yui se prolonge en colonne à l'extrémité supérieure.

Autour des tubes criblés, la gaine fasciculaire du faisceau montre une épaisseur maxima.

Lacunes grandes occupant dans les feuilles anciennes presque toute l'épaisseur de l'organe; le parenchyme se trouve alors réduit à former bordure aux faisceaux et à l'épiderme.

Slomates présentant une structure analogue à ceux de l'espace précédente, répandus sur les deux faces, à cellules annexes faisant aussi saillie au dehors de l'épiderme (fig. $24 a$ ct $b$ ) ct enclavés dans les zigzags de ce dernier.

Tige triangulaire présentant une grande ressemblance avec celle de $\mathrm{C}$. stricta, à angles plus aigus cependint que chez cette dernière espèce et doublés vers l'intérieur 
de groupes de cellules sclérenchymateuses étalés sous l'épiderme.

Faisceaux libéro-ligneux très nombreux disposés sur deux rangs, pourvus de revêtements fibreux allongès en colonnes du côté de l'épiderme et réduits à deux ou trois assises à l'extrémité intérieure.

Epiderme papilleux portant les mêmes stomates que la feuille.

Rhizomes à faisceaux concentriques dont les phloëmes présentent une structure normale.

Endoderme très fortement épaissi sur le còté interne (fig. 71) avec ponctuations, parfois double el dont les lamelles moyennes se montrent lignifiées sous l'action des réactifs.

Parenchyme lacuneux formé de cellules arrondies laissant entre elles de grands méats et richement pourvues de ponctuations (fig. 72 sect. long.)

Racine dépourvue d'une zone péricambiale entre les vaisseaux primaires et l'endoderme, lequel, comme pour le rhizome, est formé d’éléments à épaississements massifs sur le côté interne, à lumens très réduits et à lamelles moyennes lignifiées. Ces cellules sont toujours richement ponctuées et leurs poncluations sont souvent ramifiées (fig. $73 a b$ ).

Parenchyme cortical présentant l'arrangement habituel en toile d'araignée et des nodosités nombrenses.

Sous l'épiderme, anneau sclérenchymateux très déreloppé.

Considérées dans leur ensemble, les structures anatomiques des organes de végétation des trois espèces C. stricta, C. vulgaris, C. acuta, présentent entre elles à part quelques différences rle détails ayant trait à divers tissus et parties de ces plantes, tels que l'endoderme des racines et la nature des rhizomes - une grande ressem- 


\section{5}

blance, laquelle se retrouve aussi dans les caractéres extérieurs.

Nous avons ainsi un exemple où l'analyse anatomique d'espèces voisines vient confirmer les bases sur lesquelles la systématique s'est appuyée pour les grouper ensemble.

Carex montana. L [Reich. Icon. VHi, 633 (C. collina W.)]

Feuille petite à épidermes réguliers presque lisses ou ne présentant que rarement - surtout sur les bords des cellules développées en papilles.

Cellules de plissement sur plus d'un rang, celles du premier brusquement différenciées des autres cellules épidermiques par leur grandeur, radialement comprimées, au nombre de huit environ.

Epiderme infërieur complètement lisse.

Faisceaux rapprorhés de la face inférieure, entourés d'une gaine fasciculaire assez forte et d'un rang de cellules aqueuses de chaque còté.

Tissu vert formé de cellules arrondies ne renfermant pas de cellules tannifëres.

Stomates superficiels du trpe ordinaire, seulement sur la face inférieure, au milieu d'un épiderme zigzagué.

Tige nettement triangulaire, pourvue d'un épiderme dont les éléments se présentent arrangés d'une façon régulière et de forme arrondie. Quelquefois, et cela surtout au sommet des angles, on remarque qu'une ou deux cellules se sont différenciées de leurs roisines en prenant un plus grand développement, jusqu'à devenir un éperon très proéminent et très aigu, plus effilé que les crochets chez d'autres espèces fig. 74).

La tige creuse renferme des faisceaux libéro-lionneux au nombre de quinze à dix-neuf environ arrangés sur un seul rang, quelques-uns seulement, les plus gros d'entre eux, arrivent à toucher l'épiderme par leur revêtement mécanique. 
Les autres montrent sur leur face externe une gaine fitreuse plus épaisse avec tendance de cette partie à rejoindı'e par l'intermédiaire de son sclérenchyme la r'égion épidermique.

Mêmes stomates superficiels que dans la feuille. Epiderme fort, composé de cellules arrondies, régulières, à parois externes épaisses.

Rhizome solidement construit présentant à la périphérie un fort anneau de sclérencliyme dont les cellules se répartissent sur six à huit rangs d'épaisseur, renforcés ici et là par de petits groupes de fibres sclérenchymateuses sous-épidermiques. Parenchyme interne formé au contraire de cellules à parois minces, arrondies, faisant contraste arec celles de la périphérie el dont l'ensemble occupe à peu près la moitié du tissu cortical.

Le cylindre central est ici protégé par un endoderme dont les éléments plus ou moins arrondis sont épaissis sur tout leur pourtour et s'appuient sur un péricambium lignifiè à cloisons fortes, faisant fonction par endroits de double endoderme.

Faisceaux libero-ligneux collatéraux dans l'écorce, concentriques à l'intérieur du cylindre central, munis de phloëmes normaux. Ils occupent la presque totalité du cylindre et s'anastomosent souvent entre eux par leurs gaines fibreuses.

Observées en coupes longitudinales, les cellules de l'anneau sclérenchymateux se présentent sous la forme tabulaire avec parois massives, riches en ponctuations lesquelles s'étendent bien avant à l'intérieur du tissu cortical (fig. 75).

Racine pourvue aussi d'un anneau périphérique d'éléments mécaniques de six à huit assises d'épaisseur sur le côté où il est le plus développé.

Cellules du parenchyme collapsant de bonne heure entre elles. 


\section{7}

Endoderme composé de cellules ovales, règulières, relativement peu épaissies sur le côté interne.

Lames vasculaires courtes, s'avançant peu dans le parenchyme ligneux central laissant à ce dernier le champ libre; les vaisseaux primaires s'appuient ici tantôt sur un péricambium nettement développé, tantôt directement sur l'endoderme.

Carex preceox. L. Jacq. (Reich Icon. VIII, 634)

(C. verna. Vill. - C. umbrosa. Host.)

Feuille. - Epidermes d'inégale grandeur sans poils ni papilles appréciables en coupe transversale, à cellules de plissement très nettement différenciées, arrangées en éventail à parois externes fortement épaissies, à parois radiales minces, et ne croissant pas graduellement en grandeur, mais s'étalant brusquement en face de la nervure médiane (fig. 76 ).

Faisceaux libéro-ligneux au centre de la feuille entouré d'une gaine fasciculaire dont l'épaississement maximum a lieu autour des tubes criblés, pourvus à chaque extrémité de revêtements puissants, lesquels ne sont pas accompagnés de cellules aqueuses; celles-ci se montrent en petit nombre à gauche et à droite du faisceau proprement dit. Les petits faisceaux ne paraissent pas non plus être entourés de ccs mêmes cellules comme c'est le cas habituel. Tissu vert dont les lacunes sont séparées de l'épiderme par plusieurs couches de cellules sur le côté supérieur, tandis que sur l'autre, le nombre de ces couches est réduit souvent à un. Nombreux méats (cellules en entonnoir).

Stomates sur la page inférieure, à fleur d'épiderme répondant au type ordinaire de structure.

En coupe longitudinale, la feuille montre sur l'épiderme de la page supérieure quelques papilles-crochets couchées sur les autres cellules et fortement épaissies à leur extrémité (fig. 77 a). 
Tige à angles très arrondis, à épiderme fortement zigzagué, dont les cellules se développent souvent en saillies arrondies; faisceaux disposés sur un seul rangr d'après le plan habituel.

Parois externes de l'épidérme minces.

Stomates superficiels. Papilles-crochets sur les arêtes.

Phizome traçant à tissu parenchymateux muni de lacunes, lesquelles sont séparées entre elles par des colonnes de parenchyme. Ces colonnes renferment généralement un faisceau libéro-ligneux.

Les parois du tissu cortical qui par suite de collapsus et de resorption forment des lacunes, se trouvent pourvues de nodosités.

Anneau sclérenchymateux à la périphérie, formé de plusieurs couches dont les plus extérieures sont interrompues par de petits groupes de cellules lignifiées à parois très épaisses.

Endoderme composé d'éléments à lamelles moyennes lignifiées, peu accentués sur leur côté interne comparativement à ceux des autres espèces, grands et arrondis, à peu près égaux sur tout leur pourtour et s'appuyant sur une zone péricambiale dont les cellules sont de force équivalente (fig. $77 \mathrm{~b}$ ).

Faisceaux concentriques entourés de gilines fibreuses, à phloëmes présentant la structure normale et groupés à la périphérie, laissant toujours le centre du cylindre central occupé par le parenchyme ligneux seul.

Dans les jeunes rhizomes, on ne trouve ni nodosités ni lacures, tout le tissu cortical étant constitué par des cellules rondes, à parois minces, analogues à celles du tissu correspondant de la racine, la disposition radiale exceplée.

Pas de cellules tannifères ni de méats bien développés.

Racine pourvue d'un péricambium (fig. 78) existant 
aussi entre les vaisseaux primaires et les éléments de l'endoderme qui se trouvent également avoir des parois relativement minces et renfermer de l'amidon. Le tissu cortical interne tombe de bonne heure en collapsus.

Nodosités comme à l'ordinaire.

Carex humilis. Leyss. - Reich. Icon., vIII, 595.

(C. clandestino Good).

Feuille pelite présentant de grandes lacunes; l'épiderme, fortement construit sur les deux pages, offre ici et là, mais toujours rarement, une cellule développée en crochet.

Pas de cellules de plissement différenciées d'avec les autres éléments épidermiques.

Faisceaux situés à peu près au centre de la feuille, occupant avec le revêtement mécanique toute l'épaisseur de cet organe. Leur forme est en général très allongée.

Cellules aqueuses sur les flancs des faisceaux réduites à un petit nombre.

Stéréôme considérablement développé partageant ce caractère avec les gaines fasciculaires.

Vaisseaux très nombreux dans un seul el mème faisceau, disposés en deux séries contiguës.

Cônes siliceux, quelquefois très gros dans les deux épidermes. Parenchyme chlorophyllien parsemé de cellules rondes et incolores, fréquentes surtout dans le voisinage des lacunes et formant des réservoirs à tannin.

Stomates sur la page inférieure, superficiels, petits, avec chambre de respiration exiguë. Vus de face, ils se présentent dans un épiderme zigzagué et sont pourvus de très grosses gouttes huileuses. Bords supérieurs et inférieurs des cellules stomatiques fortement épaissis.

Tige triangulaire, mais dont les angles sont très arrondis, pourvus d'un épiderme portant parfois (aux angles 
surtout) une cellule développée en éperon ou en crochet à parois plus minces qu'à l'ordinaire.

Les faisceaux, au nombre d'une vingtaine, cntourés de gaines fasciculaires de peu de résistance, sont comme d'habitude, les uns marginaux avec revêtements, les autres plus intérieurs. Pour les premiers, leurs revêtements s'étalent par endroits sous l'épiderme, formant ainsi un arc de cercle mécanique qui se trouve surtout aux angles.

L'épiderme est composé de cellules régulières à parois minces munies de cônes siliceux, quadrangulaires, allongées dans le sens tangentiel.

Stomates superficiels semblables à ceux de la fenille.

Dans le milieu et le haut de la tige, les dispositions mécaniques se complètent, les revêtements libériens et les parois de l'épiderme s'accentuant davantage.

Rhizome dont tous les tissus présentent une forte structure, les cellules corticales internes participant aussi à ce développement.

Nous avons sous l'épiderme un anneau sclérenchymateux au-dessous duquel s'étend un parenchyme riche en méats intercellulaires et en amidon.

Endoderme offrant dans ses éléments un épaississement en U parfois double, et s'appuyant sur une zone péricambiale d'aspect sclérenchymateux.

Faisceaux concentriques à phloëmes normaux.

Racine fortement constituée dans toutes ses parties, pourvue d'un péricambium non interrompu par les vaisseaux primaires, d'un endoderme à cellules grandes, épaissies en U, et renfermant de l'amidon.

Parenchyme cortical se présentant en séries radiales de cellules, lesquelles ont épaissi considérablement leurs parois et collapsent plus ou moins entre elles.

Anneau épais de sclérenchyme à la périphérie. 
Carex alpestriø. All. - Reich. Icon., VIII, 630.

(G. gynobasis, Vill. - G. halleriana Asso.)

Feuille mince présentant un épiderme supérieur papilleux à grandes cellules et un épiderme inférieur lisse ; à faisceaux libéro-ligneux presque centraux, à lacunes allongées parallèlement au plan de la feuille.

Cellules de plissement sur un rang peu différenciées des autres éléments épidermiques. Stomates superficiels répondant au type ordinaire.

Gaines fasciculaires massives surtout au pourtour des phloëmes.

Parenchyme riche en tannin.

Tige triangulaire dont les angles sont plus ou moins arrondis, pleine, munie à sa périphérie d'un unique rang de faisceaux libéro-ligneux.

Ces faisceaux sont munis de forts revêtements libériens et de gaines fasciculaires très épaisses. ${ }^{1}$

Stomates superficiels semblables à ceux de la feuille.

Rhizome. présentant une structure puissante et dont le cylindre central est presque tout entier occupé par les faisceaux libéro-ligneux concentriques qui s'anastomosent par leurs gaines à la périphérie.

Parenchyme cortical très solidement bâti avec un épiderme à parois extérieures épaisses, un anneau de sclérenchỵme continu, formé de plusieurs assises renfermant des groupes d'éléments encore plus accentués.

Le tissu parenchymateux proprement dit, se divise en deux parties distinctes, l'une contiguë à l'endoderme, munie de cloisons minces et réduite à quelques couches

- Les échantillons sur lesquels les coupes ont été pratiquées provenant d'un lıerbier déjà ancien, il ne nous a pas été possible de constater d'une façon certaine la présence de cellules tannifères. 
seulement; l'autre formée de cellules dont les parois s'épaississent et montrent une tendance à devenir collenchymateuses.

Endoderme composé d'éléments très puissants (fig. 79), épaissis considérablement sur leur côté interne, nettement striés, s'appuyant sur une deuxième rangée de cellules, sinon de même forme par endroits, du moins presque aussi fortes dans leurs parois.

Cette espèce montre souvent un développement inégal dans ses tissus; la chose est facile à constater dans ce rhizome dont un des côtés présente parfois toute une zone d'éléments beaucoup plus accentués; ceci tient sans doute à ce que ces derniers sont directement opposés à certaines actions mécaniques exercées sur un des côtés seulement de la tige souterraine.

Pas de lacunes dans le parenchyme cortical, mais des méats accentués.

Racine à structure de résistance, présentant un tissu cortical dont les cellules sont pourvues de cloisons épaisses, qu'on les prenne au pourtour de l'endoderme ou à la périphérie, de sorte qu'on ne distingue pas de.différence entre les deux parties de l'écorce.

Cellules endodermiques très puissamment constituées, nettement striées, s'appuyant sur une zone péricambiale continue et lignifiée (fig. 80).

En général à moitié distance de l'endoderme et de l'épiderme toute une zone de tissu cortical tombe en collapsus, formant ainsi un anneau solide plus ou moins coloré en brun ainsi que le tissu environnant.

Carex nigra. All.

Feuille à épiderme hérissé de papilles sur les deux faces, mais d'une façon plus tranchée sur l'inférieure. 
Sur la page supérieure, les cellules sont environ deux fois plus grosses.

Appareil de plissement à jointures quelque peu collenchymateuses, brusqueınent différencié des éléments voisins.

Faisceaux libéro-ligneux rapprochès du bord, pourvus de revêtements développés en colonnes, el de gaines fasciculaires fortes et grandes, dont les cellules s'épaississent surtout à la hauteur des gros vaisseaux. Cellules aqueuses autour du faisceau seulement.

Stomates exclusivement sur la face inférieure, entourés de deux papilles qui, par leur proéminence au-dessus de l'appareil stomatique, font que celui-ci paraît situé au fond d'une sorte d'entonnoir. Vu de face, l'épiderme laisse voir une ou deux de ces papilles qui viennent se réfléchir plus ou moins sur les stomates, ou se présentent sous forme de cercles encadrés par les parois zigzaguées.

Tige triangulaire à pourtour crénelé, munie d'un épiderme à papilles ayant même aspect soil en coupe longitudinale soit en coupe transversale ; à faisceaux disposés d'après le type ordinaire, munis de revêtements en colonnes et de gaines fibreuses à peu près de force égale dans les deux rangs de faisceaux.

Gaines fasciculaires épaissies autour des phloëmes et à la hauteur des deux gros vaisseaux.

En dehor's de ces deux sortes de gaines, on trouve une troisième enveloppe protectrice formée d'éléments à parois plus minces, mais lignifićs aussi et qui sont probablement destinés à faire partie plus tard d'une gaine fibreuse, la lignification et la sclérification s'étendant dans les parties intermédiaires. Mêmes stomates que dans la feuille.

Rhizome puissamment construit, présentant à la périphéric un anneau continu de sclérenchyme (fig. 81). 
Parenchyme cortical formé de cellules disposées sans régularité.

Cylindre central recouvert d'un endoderme massif et muni de faisceaux libéro-ligneux concentriques à phlö̈mes normaux, occupant à la fois le centre et la périphérie du corps ligneux.

L'endoderme, par endroits double, est formé d'éléments épaissis fortement sur la moitié interne et laissant apercevoir facilement les différ'entes couches d'épaississernent (fig. 82). Leurs lumens sont en général assez réduits.

Racine poilue, construite d'après le type ordinaire avec anneau sclérenchymateux sous l'épiderme. Chez les racines vieilles, le tissu qui porte habituellement les nodosités disparait, de sorte qu'on obtient un parenchyme cortical plus ou moins lacuneux.

Absence complète de péricambium entre les vaisseaux primaires et les éléments de l'endoderme, qui, ici aussi (fig. 83) sont fortement épaissis sur leur moitié interne. On observe en outre que les lames vasculaires présentent dans le corps central une longueur considérable el qu'à l'extérieur de l'endoderme, il existe trois ou quatre rangs de cellules radiales à méats, lesquelles persistent toujours.

\section{Carex atrata. L.}

Feuille à épiderme supérieur lisse avec seulement quelques proéminences chez quelques-unes des cellules, lesquelles ont des parois externes épaisses et de grands lumens. L'épiderme inférieur, portant seul les stomates, se trouve au contraire être hérissé de papilles.

Cellules de plissement sur un seul rang, nettement différenciées des éléments voisins, non collenchymateuses.

Faisceaux libéro-ligneux, rapprochés du bord inférieur de la feuille, pourvus de gaines fasciculaires dont les cellules s'épaississent au maximum en regard des gros 
vaisseaux, à revêtements mécaniques très développés, étalés à l'extrémité inférieure du faisceau et allongés en colonnes à l'autre extrémité. Cellules aqueuses formant gaine autour du tissu conducteur.

Le matériel mis à notre disposition provenant d'un herbier déjà ancien, les réactions faites en vue de trouver. des cellules à tannin n'ont pas permis de conclure à la présence de cette substance.

En coupe longitudinale, l'épiderme montre ses papilles sous le même aspect.

Stomates en général intercalés entre deux de ces proéminences, au fond de la cavité formée par celles-ci (fig. 41).

Vus de face, ils présentent la structure ordinaire, dans un épiderme zigzagué et apparaissent le plus souvent flanqués de deux cercles qui représentent les papilles susdites.

Tige triangulaire à angles très aigus, à épiderme papilleux, comme celui de la feuille, présentant les mêmes zigzags, mais un peu plus épaissis quant à leur's parois, et les mêmes slomates.

A chaque angle el sur chaque côté, on observe un groupe de cellules mécaniques plus ou moins considérable.

Faisceaux libéro-ligneux nombreux au nombre d'une quarantaine environ disposés sur deux rangs comme à l'ordinaire, mais munis, surtout ceux du bords, de revêtements très puissants formant des colonnes qui portent à leur extrémité interne le faisceau lui-mème.

Les plus intérieurs sont aussi pourvus de gaines et cellules lignifiées, très épaisses sur le bout du faisceau qui regarde le centre, ces gaines lig̣neuses s'anastomosent fréquemment entre elles.

A mesure que l'on monte dans la tige, ces anastomoses s'accentuent de plus en plus, jusqu'à occuper tout le centre réservé jusqu'alors au parenchyme médullaire. 
Rhizome remarquable aussi par la solidité de ses tissus.

L'endoder'me y est for'mé par des cellules petites, il est vrai, mais très épaissies sur leur côté interne, à lumens étroits, tantôt ovales, tantôt pyramidaux ou arrondis, et s'appuyant sur une zone péricambiale à cloisons également fortes et lignifiées.

Faisceaux libèro-ligneux nellement concentriques, à phloëmes normaux, entourés chacun de gaines fibreuses formées de plusieurs assises d'éléments.

Nombreuses anastomoses à la périphérie. Au milieu du cylindre central, l'espace réservé au parenchyme ligneux se trouve très réduit par l'envahissement des faisceaux.

Parenchyme cortical composé de cellules laissant entre elles des méats, mais pas de lacunes et dont les cloisons sont plus fortes qu'à l'ordinaire.

Les cinq ou six dernières assises poussent plus loin leur développement en s'épaississant encore davantage et en lignifiant leurs cloisons de manière à former un anneau d'éléments mécaniques, sans passer cependant par la forme collenchynateuse (fig. 84). Cel anneau montre ici et là, en regard des faisceaux libéro-ligneux de l'écorce, des groupes de fibres sous-épidermiques qui communiquent entre eux par un rang d'éléments semblables courant sous l'épiderme ou servant de protection aux faisceaux corticaux de la périphérie.

Nombreux cônes siliceux dans l'assise épidermique opposée à ces tissus mécaniques (fig. $84 \mathrm{c}$.)

Racine solidement construite, présentant à la périphérie quelques protubérances papilleuses.

Péricambium constamment interrompu par les vaisseaux primaires.

Endoderme formé de cellules massives à moitié interne plusieurs fois plus épaisse que l'autre, s'appuyant à 
l'extérieur sur des séries radiales à parois plus fortes qu'à l'ordinaire et dont les éléments présentent un diamètre à peu près égal dans les quatre premières couches (fig. 85). Peu à peu celte disposition change, et l'on obtient alors, si la résorption n'a pas eu lieu, le tissu caractéristique en toile d'araignée.

A celte partie de l'écorce succède un for't anneau sclérenchymateux réparti sur environ six couches d'éléments lignifiés, lesquels précèdent une assise épidermique à parois plus minces pourvue de quelques poils.

La même remarque faite plus haut sur le rhizome de C. alpestris à propos de développement inégal survenant sur un des côtés de l'organe, s'applique aussi aux cellules endodermiques el à l'anneau sclérenchymateux de la racine qui nous occupe.

L'espèce C. Buxbaumii et celle que nous venons d'étudier, toutes deux habitant les marais, nous fournissent un exemple de deux espèces qui, bien que systématiquement voisines présentent cependant des différences de structure anatomique assez considérables.

Comparant en effet les deux analyses que nous avons faites de C. Buxbaumii et de C. atrata, nous voyons d'une part des stomates sur les deux faces, d'autre part nous constatons que ces organes sont confinés à la page inféricure de la feuille. Chez l'une des espéces les papilles sont également bien développées sur les deux épidermes; chez l'autre, c'est sur la page inférieure qu'elles se trouvent le plus richement représentées.

C'est ensuite dans les rhizomes que nous trouverons une nouvelle différence en ce sens que $C$. Buxbaumii est pourvu d'une tige souterraine stolonifère, rampante, tandis que C. atrata nous l'offre essentiellement cespiteuse. On pourriit encore trouver d'autres caractères, mais ceux 
indiqués plus haut satisfont suffisamment au but proposé et pouriont être employés avantageusement comme caractères distinctifs dans la détermination.

Carex ornithopoda W. Reich, Icon. VIII, 598.

(C. pedata. Schkhr.)

Feuille. - Epiderme de la page supérieure environ deux fois plus grand que celui de la face inférieure, composé de cellules ayant tendance à former des papilles arrondies (fig. 86.)

Cellules de plissement graduellement différenciées, nombreuses (environ une quinzaine), à parois radiales plus épaisses que d'ordinaire et à jointures peu ou pas collenchymateuses.

Epiderme inférieur lisse.

Faisceaux libéro-ligneux munis de fortes gaines fasciculaires épaissies sur tout leur pourtour, et non pas seulement en regard des phloëmes.

Cellules aqueuses le long des faisceaux et de leurs colonnes fibreuses.

En coupe longitudinale, l'épiderme de la page supérieure présente des cellules développées en saillies arrondies (fig. 86) sur le côté extérieur ; pas de poils ni de papilles en forme de crochets. Tissu vert lacuneux comme habituellement.

Revêtements fibreux puissamment développés dans les nervures principales.

Pas plus que dans l'espèce précédente, il ne nous a été possible de constater la présence de cellules tannifères.

Stomates sur la face inférieure, superficiels et répondant au type ordinaire par leur structure.

Vus de face, ils se présentent aussi enclavés dans un épiderme zigzagué, laissant apercevoir en dessous tout un 
tissu riche en méats et des chambres stomatiques assez grandes.

Tige triangulaire à angles très arrondis, dont les cellules de l'épider'me se développent en papilles aux arêtes surtout. Ces papilles prennent souvent les proportions d'un éperon allongé (fig. $87 a b, p p^{\prime}$ )

Au bas des tiges, les faisceaux libéro-ligneux ne sont séparés de leurs voisins que par l'espace lacunaire, le tissu vert étant très réduit.

Centre de la tige creux, entouré de parenchyme composé de cellules à parois minces présentant ici et là des nodosités provenant d'un collapsus.

Stomates superficiels de même forme el de même grandeur que ceux de la feuille.

Faisceaux libéro-ligneux de deux sories; les uns rapprochés du bord et munis de revêtements épais, les autres plus internes à gaines fibreuses plus accentuées sur le côté qui regarde l'épiderme.

Dans le haut comme dans le bas de la tige florifère, le nombre des faisceaux reste constant.

Rhizome à faisceaux concentriques entourés de gaines fibreuses plus épaisses sur l'un des côtés el à phloëmes formés de cellules sensiblement égales. Tous ces faisceaux sont groupés à la périphérie, laissant au milieu du cylindre central un grand espace qui en est dépourvu.

Endoderme formé d'éléments épaissis sur leurs parois internes en forme de croissant, s'appuyant sur une zone d'autres cellules à parois épaisses et à l'extérieur sur un parenchyme à méats dont les cellules n'offrent pas de disposition régulière (fig. 88.)

Anneau sclérenchymateux sous-épidermique de trois à quatre couches de profondeur.

Racine solidement bâtie, présentant ici et là, à la périphérie, une cellule allongée en poil court papilleux. 
De bonne heure, le tissu parenchymateux qui, chez les autres espéces, est muni de nodosités, disparait, ne laissant subsister que les cellules à parois épaisses el noueuses qui entourent l'endoderme et l'anneau sclérenchymateux de la périphérie.

Eléments endodermiques fortement accentués sur leur moitié interne en forme de croissant, s'appuyant sur un péricambium lignifié mais disparaissant en regard des vaisseaux primaires (fig. 185.) Les gros vaisseaux proéminent fort peu ver's le centre, terminant ainsi des lames-vasculaires très courtes.

Carex digitata K. (Reich. Icon. VIII, 599.)

Feuille absolument semblable dans la disposition de ses organes à celle du C. Ornithopoda; on peut constater cependant au point de vue des gaines fasciculaires une tendance à une structure plus forte chez ce dernier. Son habitat de stations montagneuses, quelquefois rocheuses, pourra peut-être expliquer cette petite différence.

Nous avons ici aussi un développement considérable de cônes siliceux el de groupes sclérenchymateux isolés aux deux bords de la feuille.

Mêmes stomates enclavés dans un épiderme zigazagué recouvrant un parenchyme spongieux et de grandes chambres stomatiques.

Tige de même forme, avec même arrangernent des faisceaux libèro-ligneux; mêmes grandes lacunes réduisant beaucoup de tissu vert et le refoulant ver's l'épiderme et les colonnes de stéréome; quelques papilles faisant proéminence, mais toujours rares.

Même remarque relative à l'épaississement des tissus.

La seule différence existant entre les tiges des deux espéces - différence peu inıporlante, il est vrai,--- consiste dans le fait que chez $\mathrm{C}$. digitata, les crochets manquent 
pour ainsi dire complètement, tandis que chez C. ornithopoda, ils sont déjà visibles à l'œil nu et se trouvent en grande quantité.

Rhizome semblable quant aux faisceaux qui sont concentriques, aux éléments de l'endoderme, aux tissus parenchymateux et sclérenchymateux.

Toutefois, on observe ici pour les cellules de l'endoderme une forme plus comprimée. Mais tout ceci peut dépendre de la nature même du rhizome et de l'endroit où ont été pratiquées les sections.

Dans la racine, nous retrouvons aussi une structure moins forte que celle de $\mathrm{C}$. ornithopoda, mais répondant à une organisation générale semblable.

Dans l'endoderme des deux types, nous avons des éléments considérablement épảissis sur la inoitié interne, de sorte que toute différence de structure s'efface plus ou moins complètement. Mais dans l'une des espèces, chez C. digitata, nous avons la forme caractéristique en $\mathrm{V}$ à boucle épaissic ; chez l'autre, C. ornithopoda, la boucle de l'U devenant très massive, arrive à occuper la moitié de la longueur totale de la cellule.

Les deux espèces sont pourvues d'un anneau extérieur de sclérenchyme, lequel est moins accéntué dans l'espèce qui nous occupe.

Pas de péricambium entre les vaisseaux primaires et l'endoderme.

Carex alba L. (Reich. Icon. VIII, 596.)

Feuilles petites, munies d'un épiderme à cellules larges, lisses, plus grandes sur la page supérieure que sur l'inférieure. En coupe transversale, elles paraissent dépourvues de poils ou de papilles.

Appareil de plissement formé par un groupe de sept à huit éléments disposés sur un seul rang et dont les plus 
grands arrivent à occuper la moitié de l'épaisseur totale de la coupe.

Les faisceaux sont disposés au bord de la feuille avec revêtements mécaniques; les plus petits occupant le centre de l'organe ; tous sont pourvus de gaines fasciculaires fortes.

Cellules aqueuses peu nombreuses, ne formant pas colonne entre l'extrémité du faisceau et l'épiderme.

Au bord des feuilles, le stéréome se développe considérablement sous forme de cellules fibreuses s'étalant en plusieurs couches sous l'épiderme.

Lacunes grandes, allongées tangentiellement à la surface. On y trouve presque toujours des débris de cellules résorbées.

Parenchyme chlorophyllien pourvu d'espaces intercellulaires (ou bifurcations) (fig. 89) ; - pas de cellules tannifères.

- Stomates grands, allongés, dans un épiderme zigzagué, superficiels, à épaississements sur la paroi dorsale des cellules de fermeture, de sorte que les lumens prerinent la forme de deux croissants qui se touchent dos à dos (fig. 21.) Chambre respiratoire très grande. Ces stomates sont en général un lieu de dépôt pour des gouttes huileuses, solubles dans l'alcool et donnant toutes les réactions propres à ces sortes de composés.

Tige creuse, très arrondie dans le bas, présentant des faisceaux pour la plupart marginaux, et munis de revêtements qui ne s'étendent pas sous l'épiderme, alternance régulière par ordre de grandeur.

Epiderme lisse, portant des stomates semblables à ceux de la feuille, encadrés dans un épiderme zigzagué.

Rhizome à faisceaux concentriques. Endoderme cornposé d'éléments épaissis en U, s'appuyant à l'intérieur sur une zone de cellules à parois fortes (fig. 90). Parenchyme 
cortical à méats intercellulaires, offrant à la périphérie des groupes fibreux șous-épidermiques. En somme, structure ordinaire, sans particularitè bien intéressante.

Racine pourvue d'un péricambium entre les vaisseaux primaires et les cellules de l'endoderme. Ces dernières sont pourvues de parois internes massives, tandis que la partie externe des cellules reste relativément mince (fig. $91 a b$ ); lamelles moyennes fortement lignifiées. Nodosités dans la région collapsée du tissu cortical.

Dans le parenchyme cortical contigu à l'endoderme se trouvent quelquefois des cristaux qui présentent les caractères de l'oxalate de chaux.

Carex mitida. Host. (Reich. Icon. VlII, 641.)

Feuille remarquable par son peu d'épaisseur, à épidermes lisses, réguliers, à cellules de plissement quelque peu différenciées de celles de l'épiderme supérieur, à lacunes allongées parallèlement à la surface.

Faisceaux presque centraux, munis à leur extrémité de larges revêtements et dont les gaines s'épaississent principalement autour des phloëmes.

Parenchyme pourvu de cellules tannifères; stomates superficiels répondant au type ordinaire de structure, répartis sur lit face inférieure seulement, et dans un épiderme zigzagué.

Tige triangulaire pleine dans le bas, à épiderme lisse et régulier, vallonnée, à contours arrondis, présentant des faisceaux libéro-ligneux arrangés sur deux rangs d'après le plan habituel, à faisceaux libériens très développés, portés sur de hautes colonnes de stéréome et alternant assez régulièrement entre grands et petits.

Ces faisceaux, avec leurs gaines fibreuses épaisses, présentent donc un aspect allongé dans le sens radial; gaines fasciculaires à éléments quelque peu épaissis sur les flancs 


\section{4 '}

du faisceau en regard des cellules aqueuses, lesquelles se continuent le long des colonnes.

Stomates superficiels pourvus de lumens étroits.

Traitées par les réactifs du tannin, les sections de la tige présentent les réactions caractéristiques pour celte substance. Les cellules tannifères se trouvent, comme d'habitude, disséminées dans le tissu vert, mais on peut remarquer en outre la présence assez constante d'une de ces cellules de chaque côté d'un faiscean, et cela plus ou moins dans le voisinage de l'épiderme (fig. 92 a.)

Rhizome solidement construit, pourvu d'un épiderme à parois épaisses, contigu à un anneau de six à sept couches d'éléments sclérenchymateux de forme polygonale. A cette couche en succède une autre formèe de cellules arrondies à parois aussi épaisses et lignifiées qui se continuent jusqu'à l'endoderme et dont les plus extérieurès collapsent entre elles, tout en acquérant des nodosités. Le tissu corlical dans son ensemble, occupe dans ce rhizome, par rapport au cylindre central, un espace très réduit.

Faisceaux groupés à la périphérie, laissant le centre dépourvu de tissus conducteurs, nettement concentriques, à phloëmes normaux et pourvus de gaines fibreuses très épaisses s'anastomosant entre elles.

Eléments endodermiques quelquefois doubles, arrondis, à parois presque également épaissies sur tout leur pourtour, et s'appuyant sur une zone de cellules péricambiales à parois fortes.

Petits faisceaux collatéraux dans le parenchyme.

Racine dont le parenchyme cortical est formé de cellules ả parois fortes disposées radialement, présentant des renflements noueux et collapsant entre elles pour épaissir ensuite considérablement leurs parois sous l'épiderme. La forme typique en toile d'araignée disparaît. 
Cellules de l'cndoderme régulières, grandes, presque également épaissies sur tout le pourtour (fig. 92 b.) Péricambiun continu, bien développé. Lames vasculaires plutôt courtes.

Cette racine, avec son assise péricambiale bien déreloppée est des plus favorables à l'étude de l'insertion des radicelles, qui, ainsi qu'on l'a vu plus haut, procèdent par division de cellules péricambiales vis-à-vis des vaisseaux primaires (fig. 93.)

Carex glauen. L. - Reich Icon. VIII. 648.

(C. Recurva Huds.)

Feuille. - Epidermes papilleux, surtout sur la page (fig. 99-96) inférieure, la supérieure n'offrant que quelques crochets (fig. 95) ou des saillies de cellules arrondies, mais toujour's rarement. Parois des cellules épidermiques massives renfermant - sur la face supérieure entre les diverses couches de cuticularisation, des dépôts d'un corps cireux biréfringent, soluble dans l'alcool bouillant (fig. 98 et 105).

Cellules de plissement sur deux rangs, collenchymateuses; celles du premier, au nombre d'une douzaine, font un contraste brusque par leur grandeur arec les cellules voisines, et présentent ici et là, dans leurs parois radiales, quelques ponctuations simples. Le second rang est formé d'une rangée d'éléments d'une forme plus ou moins carrée et de volume plus petit (fig. 104).

Stomates enfoncés profondément, lcs cellules épidermiques voisines faisant saillie au-dessus de l'appareil stomaticue en formant une sorte de cheminée à canal plus ou moins long, qui va s'élargissant de plus en plus à mesure qu'il approche de l'ouverture. Chambre sousstomatique en général étroite. Epaississement considérable 
des cellules de fermeture, ce qui amène un rétrécissement prononcé de leurs lumens (fig. $33 a$ et 34).

En coupe longitudinale, la feuille nous montre un épiderme hérissé de papilles à sommet arrondi dans lequel les stomates se présentent sous un aspect particulier. On a alor's un corps allongé renflé aux deux extrémitès, surmonté de trois cercles plus ou moins parfaits, formés par les sinuosités épaissies des cellules voisines, lesquelles présentent comme à l'ordinaire des parois zigzaguees (fig. 97).

Tissu parenchymateux richement pourvu de cellules tannifères présentant une disposition régulière; on les trouve en effet isolées au nombre de deux à trois ou plus, à droite et à gauche des colonnes de stéréome, quelquefois sous l'épiderme, le plus souvent à quelque distance de celui-ci (fig. 105). Faisceaux libéro-ligneux entourés d'une gaine forte très épaissie le long des phloëmes et dans laquelle les éléments vont en diminuant de force et en s'arrondissant autour du xylème; revètements mécaniques qui, à l'extrémité supérieure, sont développés en colonnes et accompagnés par les cellules aqueuses.

Lacunes de formes irrégulières entourées d'un tissu vert dont les cloisons offrent, surtout au voisinage de l'épiderme inférieur, de nombreuses bifurcations de membranes, lesquelles se trouvent aussi bien sur les cloisons tangentielles que sur celles qui sont perpendiculaires à la surface de la feuille.

Tige triangulaire à épiderme de force moyenne, portant des stomates enfoncés comme ceux de la feuille, et ne présentant dans ses parties inférieures que quelques saillies papilloïdes, qui vont en s'accentuant à mesure que l'on remonte vers l'épi et sont parfois très développées au sommet des arêtes. 


\section{7}

Faisceaux libéro-ligneux très développés, souvent très longs et dont la gaine montre un épaississement maximum sur le pourtour des phloëmes. Ils sont arrangés sur deux rangs, les uns marginaux avec revêtements fibreux, les autres plus intérieur's protégés par une gaine de sclérenchyme plus épaisse sur le côté qui regarde l'épiderme, Le tannin se retrouve ici aussi dans les mèmes cellules rondes, incolores, situées sur les flancs des faisceaux au voisinage de l'épiderme. Très souvent l'on trouve sur les sections transversales d'un côté du faisceau, une seule de ces cellules tannifères et de l'autre deux, placées l'une au-dessus de l'autre (fig. 108).

Rhizome relativement faible de construction; on ne trouve, en effet, à lả périphérie qu'un petit anneau de cellules sclérenchymateuses à cloisons minces ponctuées, recouvertes par un épiderme à éléments amincis dans leurs parois, aplatis et portant de nombreux cônes de silice.

Cet anneau, composé d'une à deux assises de cellules polygonales, est interrompu par endroits par des éléments du parenchyme cortical, qui peuvent devenir collenchymateux.

Ce dernier est lui-même formé de cellules arrondies, arrangées sans ordre, ne contenant pas de tannin et s'appuyant sur un endoderme à éléments réguliers, épaissis en forme de croissant.

Le cylindre central renferme à la fois les deux espèces de faisceaux avec toutes les formes de transition, depuis la structure collatérale jusqu'à la concentrique avec prédominance très marquée de cette dernière.

Ils sont répartis sur deux ou trois rangs, laissant le centre occupé par le parenchyme et sont entuurés de gaines fibreuses minces s'anastomosant entre elles à la périphérie. 
Tout le tissu cortical extérieur est richement pourvu de ponctuations, bifurcations de membranes et méats intercellulaires proprement dits.

Chez les rhizomes âgès, l'anneau sclérenchymateux se montre formé d'éléments plus résistants, généralement colorés, faisant contraste marqué avec les cellules minces et tabulaires de l'épiderme. Lacunes plus ou moins prononcées.

Racine pourvue d'un fort anneau sclérenchymateux à éléments épaissis répartis sur plusieurs couches, limité à l'extérieur par des cellules à parois moins épaisses, cloisonnés tangentiellement.

Tissu cortical interne en toile d'araignée.

Cylindre central recouvert d'un endoderme épaissi en $\mathrm{U}$ et dont les cellules ont une forme régulière.

Zone péricambiale composée d'èléments lignifiés et continue entre les vaisseaux primaires et l'endoderme.

Carex maxima. Scop.- - Rei.h. Icon. VIII. 604.

$$
\text { (C. Pendula Huds.) }
$$

Feuille à épidermes formés de cellules de grandeur à peu près égale sur les deux faces; sur la page supérieure, leurs parois externes sont lisses, sur l'inférieure elles sont développées en papilles nombreuses (fig. 109).

Cellules de plissement sur plus d'un rang et nombreuses, celles qui font partie de l'épiderme ayant la forme ordinaire comprimée, les couches inférieures étant composées d'éléments d'aspect parenchymateux et plus petits.

Faisceaux libéro-ligneux disposés comme dans la fig. 109, accompagnés sur leurs côtés et à leur extrémité par un système de cellules aqueuses très développé. Il en est de même des colonnes de stéréôme qui les protègent. Les gaines fasciculaires sont fortes, présentant une épaisseur maxima sur les côtés où manquent des cellules 
fibreuses et où le faisceau se trouve, par conséquent, en rapport direct avec les cellules aqueuses. Grandes lacunes dans le mésophylle.

Stomates petits entourés de grandes cellules annexes dépassant de beaucoup en longueur les cellules stomatiques; paraissant tantôt à niveau de l'épiderme, tantôt enfoncées entre deux papilles, pourvus de chambres exiguës. Ils sont, comme à l'ordinaire, enclavés dans un épiderme zigzagué dont les cellules portent souvent un cercle à contours épaissis correspondant à une papille (fig. $25 a b$ ).

Tige triangulaire fortement triquètre, dont les angles sont doublés sous l'épiderme de groupes fibreux, et dont les faisceaux libéro-ligneux, sur deux rangs, alternent avec les lacunes.

Epiderme composé de cellules régulières, dont quelques-unes seulement sont développées en papilles semblables à celles de la face inférieure de la feuille. Stomates comme dans la feuille.

Stéréome puissamment développé sur le côté externe des faisceaux; ceux-ci sont eux-mêmes entourés d'une gaine fasciculaire de cellules arrondies, à membranes lignifiées, également épaissies sur tout le pourtour du faisceau.

Cellules tannifères nombreuses dans le parenchyme.

Rhizome gros et épais fortement constitué, surtout à l'intérieur du cylindre central où les faisceaux sont entourés de gaines fibreuses à plusieurs couches d'épaisseur.

Ceux-ci sont pour la plupart concentriques, mais offrent cependant quelquefois des formes de transition à la collatéralité (fig. 111).

Endoderme à parois internes massives, dont les éléments de même forme que ceux de C. alba, sont richement 
ponctués el doublès en dedans d'une couche d'éléments péricambiaux à parois épaisses. Leurs lamelles moyennes sont fortement lignifiées.

Parenchyme cortical formé de ccllules rondes, assez riches en amidon, disposées en files radiales, laissant entre elles des lacunes allongées radialement et renfermant encore des restes de tissu en voie de résorption.

Racine dépourvue d'assise péricambiale entre les éléments de l'endoderme et les vaisseaux primaires. Le tissu cortical y est pourvu d'un grand nombre de nodosités (fig. $114 a, b, c, d, e, f$ ). Du reste même aspect, même répartition des ponctuations que chez $C$. acuta, avec lequel, au point de vue anatomique, l'espèce qui nous occupe, offre de nombreux points de ressemblance.

Anneau de sclérenchyme puissant formé de six à sept couches d'éléments de formes polygonales (voir fig. 114 A).

Cellules endodermiques à parois massives, épaissies en $U$, comprimées entre elles, et sculptées de nombreuses ponctuations (fig. 112 et 113).

Carex strigosa. Huds. - Reich. Icon. VIII, 602.

Feuille molle à épiderme lisse sur les deux faces, à cellules beaucoup plus grandes sur la face supérieure, mais qui en regard des principaux faisceaux vasculaires deviennent plus petites en même temps qu'elles s'enfoncent dans les tissus.

Cellules de plissement nettement différenciées, comprimées radialement entre elles et non collenchymateuses.

En coupe longitudinale, la feuille présente des méats profonds (cellules en entonnoir).

Comparée à celle de l'espèce précédente, la structure de cet organe nous apparait beaucoup moins capable de résistance. 


\section{1}

Nous avons en effet des revêtements et des colonnes d'éléments mécaniques bien moins fortement développés. Les faisceaux eux-mêmes appuyés contre l'épiderme inférieur ne possèdent pas de gaines fasciculaires très accentuées; comme dans la plupart des espèces. Comme à l'ordinaire, une ceinture de cellules aqueuses entoure les tissus conducteurs.

Parenchyme riche en méats intercellulaires et en bifurcations de membranes, de même qu'en cellules tannifères (fig. 115).

Il est facile de voir dans cette espèce que primitivement, avant la formation des lacunes qui sont ici dirigées dans le sens du grand diamètre de la section transversale et entourées de plusieurs couches de cellules assimilantes, le tissu qui remplissait ces cavités était aussi pourvu de cellules tannifères, lesquelles, au moment de la résorption, persistent et se trouvent entrainèes et acculées au bord de la lacune par le retrait du tissu résorbé.

Stomates sur la face inférieure à fleur d'épiderme ayant même structure que ceux de C. hordeïstichos; toutefois, on remarquera ici que les parois des cellules annexes avancent moins dans la cavité stomatique. De face, leur forme et leur structure sont semblables.

Tige triangulaire à angles arrondis, présentant un rang de faisceaux munis de revêtements de force moyenne.

Quelquefois, sur les coupes transversales pratiquées dans le bas de la tige, on trouve un deuxième rang de faisceaux plus intérieurs.

Epiderme lisse à parois plus minces que d'ordinaire, il en est de mêmes des gaines fasciculaires.

Mêmes stomates superficiels.

Rhizome intéressant par la grande quantité de résarvoirs à tannin qu'il renferme, surtout dans le parenchyme 
cortical. Faisceaux concentriques à phloëmes composés de cellules sensiblement égales.

Endoderme dont les éléments varient quant à la forme, suivant les parties du rhizome où ils se trouvent.

En somme, structure analogue dans son ensemble à celle de C. punctata (voir plus loin page 156), moins toutefois la formation du collenchyme qui a lieu chez cette dernière espèce.

Racine dont l'endoderme présente un épaississement en U (fig. 116). Zone de péricambium lignifié, disparaissant ici et là entre les vaisseaux primaires et l'endoderme.

Nodosités et arrangement des cellules corticales comme à l'ordinaire, lorsqu'il n'y a pas de collapsus ou résorption.

La couche de cellules la plus externe à la périphérie, chez les racines qui ne sont pas trop vieilles, porte quelques poils persistants.

Carex distans. L. - Reich. Icon. VIII, 622.

Feuille dont l'épiderme varie suivant la page considérée; la supérieure présentant des cellules trois fois plus grandes environ que celles de l'inférieure, à parois externes fortes.

Cellules de plissement moyennement différenciées disposées sur un rang. Quelques-uns des autres éléments de l'épiderme montrent une tendance à un développement papilleux.

Faisceaux libéro-ligneux occupant le centre de l'organe, entourés d'un rang de cellules aqueuses à grands lumens, flanqués à chaque extrémilé de revêtements fibreux allongés en colonnes qui traversent toute l'épaisseur entre les deux épidermes, et dont les gaines fasciculaires offrent une épaisseur maxima autour du phloëme.

Tissu parenchymateux riche en méats el en bifurcations de tous genres (fig. 117, 118, 119). 


\section{3}

Lacunes entourées de plusieurs couches de cellules assimilantes.

En coupe longitudinale, on retrouve les mêmes méats et bifurcations, el de plus, on peut constater que ces dernières sont orientées dans deux directions perpendiculaires entre elles, dans le sens longitudinal et dans le sens transversal.

ll apparaît aussi, soit sur les coupes tangentielles, soit sur celles dirigées longitudinalement, un grand nombre de grosses cellules incolores arrondies ou quelque peu allongées, toujours dépourvues de chlorophylle, el qui sunt des cellules tannifères (fig. 120).

Stomates superficiels à grandes chambres respiratoires, à becs prononcés, intercalés dans un épiderme zigzagué (fig. 37, 38 b et 122).

Tige triangulaire à parois extérieures fortes, à épiderme lisse portant des stomates superficiels et laissant aussi dans son tissu assimilateur une quantité de cellules tannifères (fig. 121).

Faisceaux libéro-ligneux sur deux rangs pourvus de revêtements larges mais cour'ts et de gaines fasciculaires très accentuées autour des phloëmes.

Rhizome cespiteux à endoderme épaissi en croissant (fig. 123,), à faisceaux concentriques dont le phloëme est composé de cellules sensiblement égales en grandeur.

Le tissu parenchymateux cortical, riche en méats, présente trois régions, l'une contiguë à l'endoderme, dont les éléments sont pourvus de cloisons minces, renfermant ici el là une cellule ronde, incolore et cn apparence vide, semblable à une petite lacune à bords bien définis $(c)$.

Pelı à peu ce tissu change d'aspect en passant à la seconde région, laquelle est formée par du collenchyme à parois ponctuées plus fortes el qui ne laissent plus 


\section{4}

entre elles de méats. La troisième, qui forme le tissu de la périphérie, est composée d'éléments plus ou moins hexagonaux à grands lumens et à parois plus minces, (roir fig. 139).

Les cellules rondes, lacunoüles, que nous voyons figurer dans la premières de ces régions, sont des réservoirs à tannin que l'on reconnaît aisément par la réaction qu'ils donnent presque instantanément par l'acide osmique, le bichromate de potasse et le chlorure ferrique. On les trouve aussi, mais en plus petit nombre, dans le cylindre central (fig. 124).

En coupe longitudinale radiale, les éléments du parenchyme ligneux montrent une infinité de ponctuations sur leurs parois, de sorte que, vus de face, ils paraissent absolument criblés (fig. 125).

Racine de structure habituelle où le péricambium se trouve interrompu la plupart du temps par les vaisseaux primaires. Cellules endodermiques épaissies en croissant, (fig. 126) s'appuyant à l'extérieur sur un parenchyme à parois épaisses qui ne tarde pas à se transformer en un tissu moins résistant dont les cellules collapsent entre elles langentiellement. .

Celte espèce constitue un des cas les plus faciles à observer sous le rapport de la présence et de l'absence simultanées d'assise péricambiale entre les vaisseaux primaires et l'endoderme.

C. depnupernta. Good. (Reich. Icon. VIII, 625.)

Feuille pourvue d'un même épiderme que dans l'espèce précédente, à cellules de plissement semblablement disposées, ayant même tendance à former des papilles sur la page supérieure.

Faisceaux au centre de la feuille, entourés de cellules 


\section{5}

aqueuses; mème armature d'éléments fibreux; gaines fasciculaires plus épaissies autour du phloëme.

Absence complète de cellules tannifères.

Stomates superficiels, construits sur le même type que celui de l'espèce précédente (fig. 26).

Tige à angles arrondis dans le bas, présentant des faisceaux disposés sur un seul rang en alternance arec de plus petits et les lacunes, et offrant une structure de moyenne résistance.

Cellules de l'épiderme allongées tangentiellement, portant des stomates superficiels et, pát'-ci par-là, une cellule développée en papille bouclée.

Gaine fasciculaire épaissie principalement sur le pourtour des phloëmes.

Il y a donc, à part l'absence de cellules tannifères, ressemblance très grande entre les tiges des deux espèces.

Il en est de même pour le rhizome au point de rue de l'aspect général. Nous avons ici aussi des faisceaux concentriques pourvus de phloëmes analogues ef de cellules endodermiques semblables.

On y trouve aussi le tissu cortical divisé en trois régions, mais comme pour la feuille, celui-ci ne renferme pas de cellules tannifères.

Dans la racine, nous trourons un second caractère distinctif en ce sens que l'assise péricambiale, toujours lignifiée, y est continue sur tout le pourtour interne de l'endoderme sans être interrornpue par les vaisseaux primaires. Quelquefois celte assise de péricambium se montre double (fig. 129).

On observe de plus une tendance de celte espèce à avoir un plus grand nombre de vaisseaux primaires que C. distans.

Examinées à un fort grossissement, les cellules endodermiques présentent des ponctuations nombreuses et une stratification très apparente. 
Carex punctata. Gaud.

Feuille à épiderme lisse, à cellule de plissement sur un seul rang, présentant la mêrne disposition générale des faisceaux que les deux espèces précédentes.

Parenchyme montrant de nombreux mėats et des bifurcations de cloisons, dans le mésophylle principalement (fig. 130). Les cellules tannifères sont ici très nombreuses, surtout au pourtour des lacunes el aux bords des feuilles, se présentant tantôt sous une forme ronde en coupe longitudinale, tantôt allongées dans le sens parallèle à la nervure médiane (fig. 131).

Stomates de même aspect et de même structure que chez $\mathrm{C}$. distans et $\mathrm{C}$. depauperata, enclavés dans un épiderme zigzagué.

Tige triangulaire à angles arrondis, à épiderme lisse portant des stomates superficiels, pourvue (fig. 132) de faisceaux disposés sur deux rangs (ceux de la périphérie munis de revêtements fibreux formant colonnes) et dont les gaines présentent leur épaisseur maxima au pourtour des phloëmes.

Cellules tannifëres très répandues dans le parenchyme et principalement dans la région interlacunaire (fig 132).

Rhizome à faisceaux concentriques entourés d'une forte gaine d'éléments mécaniques.

Endoderme fortement ponctué et épaissi en forme de croissant (fig. 133).

Comme dans les deux espèces précédentes, nous avons en dehors de l'endoderme, un parenchyme à méats (fig. 134) dont les cloisons, à mesure qu'elles s'approchent de la périphérie, se transforment d'abord en tissu collenchymateux, puis en un anneau de sclérenchyme interrompu par endroits par un groupe de fibres à parois épaisses et lignifiées (fig. 139). 
Cellules tannifères présentes aussi, mais beaucoup plus rares. Nombreuses poncluations et bifurcations dans le tissu cortical.

Racine dont l'endoderme est composé d'éléments semblables à ceux qu'on trouve chez $C$. distans, à péricambium la plupart du temps nul entre les vaisseaux primaires et l'endoderme (toutes les coupes que nous avons pratiquées dans ces racines ne nous ont montré qu'un seul endroit où la cellule péricambiale existât en r'egard du vaisseau primaire).

Anneau sclérenchymateux solide sous l'épiderme.

Comparées ensemble, les structures de ces trois espèces nous révèlent de grandes ressemblances existant surtout entre C. distans et $\mathrm{C}$. punctata.

Chez C. depauperata, ces rapports ne sont plus si évidents; d'une part, à cause de la disparition des cellules tannifëres, et de l'autre, par suite de la présence d'un péricambium continu dans la racine.

Le premier de ces caractères peut n'avoir pas d'importance el dépendre de plusieurs conditions de végétation, mais pour le second, il n'en est plus de même.

La structure plus forte de C. distans et de C. punctala peut s'expliqucr peut-être et probablement par le fait de l'habitat.

Tous deux sont des espèces à demi-marécageuses, tandis que $C$. depauperata ne se rencontre que dans les bois, c'est-à-dire dans des stations abritées où une structure de résistance ne trouve plus guère sa raison d'être.

Carex Dederi. Ehrh. (Reich. Icon. 652.)

Feuille dont l'épiderme est formé de grandes cellules sur la page supérieure, landis que sur l'autre, on ne trouve que des cellules plusieurs fois plus petites. Ici et 


\section{8}

là, on peut remarquer une tendance de quelques-unes des grandes à dépasser le niveau de leurs voisines.

Appareil de plissement différencić graduellement sans jamais cependant arriver à un diamètre qui fasse un contraste très marqué.

Faisceaux libéro-ligneux rapprochés du bord, entourés d'une gainc également forte sur tout le pourtour, munis de revêtements libériens et de cellules aqueuses.

Lacunes allongées, à peu près centrales par rapport au tissu vert, lequel renferme de grands méats et de nombreuses bifurcations.

Stomates sur la page inférieure, plutôt petits, du type ordinaire, mais dont les cellules annexes, arrondies et spacieuses, proéminent considérablement dans le tissu vert. En coupe longitudinale, ils se présentent comme à l'ordinaire enclavés dans un épiderme zigzagué (fig. 18).

Le tissu du mésophylle est ici imprégné d'un corps huileux invisible ordinairement, mais qui devient apparent et d'un beau jaune, lorsqu'on traite les coupes longitudinales par l'acide chlorhydrique. Ce corps se trouve alors séparé et s'agglomère en gouttelettes à l'intérieur des tissus.

Tige triangulaire à faisceaux libéro-ligneux sur deux rangs alternant par grandeur plus ou moins régulièrement, pourvus de fortes gaines et de revêtements mécaniques plus épais sur le côté externe. Dans les éléments épidermiques qui font vis-à-vis à ce stérẻome, se trouvent des cônes siliceux bien apparents et réguliers de forme.

Epiderme lisse portant les mêmes stomates que la feuille.

Dans le haut de la tige, les lacunes sont également développées.

Pas trace de tannin, comme dans l'organe précédent. 
Rhizome à endoderme épais (fig. 135) à faisceaux concentriques disséminés, à phloëmes normaux, et dont le tissu parenchymateux cortical, riche en méats et bifurcations (fig. 13 b) vient se terminer sous l'épiderme en collenchyme, puis en sclérenchyme.

Nombreux faisceaux collatéraux dans ce même parenchyme, entourés eux aussi de gaines fibreuses arrondies; ceux de ces faisceaux qui sont situés à la périphérie envoient ver's l'anneau sclérenchymateux un prolongement de leur gaine.

Racine dont les éléments sont construits solidement, dépourvue d'une assise de péricambium entre les vaisseaux primaires et l'endoderme. Ce dernier est composé de cellules ėpaissies en U, jusqu'à environ la moitié de la longueur totale, l'autre moitié, l'extérieure, resıant relativement mince (fig. 137.)

Dans le tissu cortical moyen, on rencontre un grand nombre de nodosités provenant du collapsus des parois tangentielles qui compriment les parois radiales et les obligent à se replier sur elles-mèmes (fig. 138.)

Carex Sylvatica. Huds. - Reich. Icon. VIII. 603.

Feuille à épiderme lisse, formé sur la face supérieure de cellules arrondies à parois externes rarement prolongées en saillies, sauf en regard des nervures principales.

Cellules de plissement assez brusquement différenciées, disposées sur un rang et se confondant bientôt arec la colonne de cellules aqueuses de la nervure médiane, de sorte qu'on obtient, au-dessus de celle-ci, tout un tissu formé de cellules incolores à parois minces. Elles se trouvent au nombre de yuinze environ, comprimées entre elles de façon à prendre peu à peu une forme quadrangulaire.

Système de cellules aqueuses très développé; elles entou- 
rent chaque faisccau sur les côtés et se continuent à son extrémité supérieure en colonnes interrompues, dans les plus gros, par quelques éléments fibreux.

Mésophylle riche en méats intercellulaires et en cellules en entonnoir.

Crochets nombreux dians la partie supérieure des arètes de la feuille.

Stomates dont la structure répond au type habiluel à niveau de l'épiderme inférieur.

Tiges à arêtes scabres dans la partie supéricure, triangulaire, à épiderme lisse et vallonné, présentant des faisceaux en grand nombre sur plus d'un rang, portés ceux du bord - sur des colonnes fibreuses allongćes; gaine s'épaississant surtout à l'entour du phloëme.

Stomates superficiels semblables à ceux de la feuille.

Rhizome dont les faisceaux concentriques, s'anastomosent à la périphérie du cylindre central et occupent presque toule la surface de celui-ci. - ...Gaines fibreuses présentant leur épaisseur maxima sur le côté qui regarde le centre. Parenchyme comme à l'ordinaire, composé de cellules rondes, passant à la forme sclérenchymateuse à la périphérie.

Eléments de l'endoderme épaissis en croissant, s'appuyant sur une zone de péricambium assez lignifiée et assez fortement construite.

Dans le tissu à la périphérie du rhizome, où le cloisonnement a lieu tangentiellement, la dernière assise des cellules qui fait fonction d'épiderme, renferme quelques cônes siliceux en regard des groupes fibreux du sclérenchyme.

Racine présentant la structure habituelle, munie à son pourtour d'un anneau sclérenchymateux dont les éléments ne présentent pas l'aspect fort des autres espèces.

Endoderme composé d'éléments à épaississement en U, 
s'appuyant aussi sur un péricambium á parois très accentuées et lignifiées, interrompu sur presque tout son pourtour par les vaisseaux primaires dont les lames vasculaires s'avancent très peu ver's l'intérieur.

Carex Pseudo-Cyperus. L. - Reich. Icon. VIII CCLXXV.

Feuille grande, présentant dans son état de développement complet de grandes lacunes et un tissu assimilateur très réduit, qui se rassemble antour de l'épiderme et autour des faisceaux libéro-ligneux, lesquels jouent ici un véritable rôle de soutien, quoique relativement petits par rapport à la grandeur de la feuille (fig. 140.)

Leurs colonnes d'éléments mécaniques traversent ici toute l'épaisseur de l'organe et sont accompagnées de cellules aqueuses, ainsi que les flancs mêmes du faisceau. Gaines fortes.

Epiderme composé de cellules relativement petites, arrondies, à parois extérieures épaissies, lisses, c'est-àdire dépourvues de poils ou de papilles, à cellules de plissement peu accentuées et différant peu des autres éléments èpidermiques (fig. 141.)

Stomates superficiels sur les deux faces, à becs prononcés, à lumens étroits, allongés, répondant au type ordinaire de structure, dans un épiderme zigzagué.

Dans le tissu parenchymateux central, se laissent apercevoir de nombreuses bifurcations de membranes cellulaires, très apparentes surtout en coupe longitudinale.

L'orientation de ces dernières est toujours la inême, c'est-à-dire qu'elles se trouvent toutes dans des parois transverses el surtout au voisinage de l'épiderme inférieur (fig. 142.)

Tige triangulaire à angles un peu plus arrondis dans 
le bas, à surface lisse, présentant des faisceaux disposés sur deux rangées, qui font ici aussi l'office de colonnes et sont séparés seulement par les lacunes, les cellules aqueuses et quelques éléments du tissu assimilateur. Leur's revêtements mécaniques sont aussi passablement développés.

Comme dans l'espèce. C. vesicaria (voir plus bas), on observe ici et là une tendance des gaines fibreuses des faisceaux à se prolonger à la rencontre les unes des autres, mais on n'a jamais la formation de ponts comme chez ce dernier, par contre, on trouve fréquemment une anastomose entre deux faisceaux voisins, dans le second rang, par leurs gaines fibreuses. Mêmes stomates et même structure interne des faisceaux que dans la feuille.

Rhizome cespiteux à endoderme massif dont les éléments présentent le même aspect que chez G. Muricata, mais avec un épaississement en $U$ plus considérable (fig. 143 a.)

Faisceaux concentriques pourvus de fortes gaines fibreuses. Parenchyme cortical à parois minces avec ponctuations, allant s'épaississant vers la périphérie. Méats et bifurcations nombreux. Cellules rirhes en amidon.

Le tissu cortical extérieur à l'endoderme est constitué par des éléments à parois minces et pourvues de nombreux méats intercellulaires, visibles principalement en coupe longitudinale (fig. $143 \mathrm{~b}$.)

Racine le plus souvent dépourvue de péricambium entre les vaisseaux primaires et l'endoderme.

Les cellules de ce dernier sont épaissies comme dans la plupart des autres espèces, mais beaucoup moins comprimées entre elles, leur plus grand diamètre étant au contraire dirigé tangentiellement et non dans le sens du rayon (fig. 144.)

Assise épidermique quelque peu papilleuse. 
Carex Riparia. Curt. - Reich. Icon. VIII, 647.

(C. crassa. Ehrh.)

Feuille à épiderme fort, présentant seulement quelques papilles sur la page supérieure, tandis que sur l'inférieure elle en est richement pourvue. Plus amples mais moins hautes sur la première de ces faces, elles deviennent sur la seconde plus étroites, plus allongées et si nombreuses qu'elles donnent un aspect bleuâtre à ce còté de la feuille (fig. 145, 146).

La disposition générale des faisceaux libéro-ligneux est semblable à celle de C. pseudo-Cyperus; on les trouve aussi doublés dans toute leur longueur - y compris les colonnes de stéréorne - d'un rang de cellules aqueuses d'un diamètre considérable.

Gaines fasciculaires très fortes à parois internes très épaissies Cellules de plissement, grandes, bien différenciées, disposées sur plus d'un rang, à jointures quelque peu collenchymateuses, les plus extérieures très allongées, les autres diminuant de grandeur à mesure qu'elles s'approchent de la nervure médiane (fig. 148).

Tissu parenchymateux pourvu de cellules tannifères, riche en bifurcations, visibles principalement en section longitudinale.

Lacunes grandes, tendant aussi à refouler le tissu assimilateur contre l'épiderme et les faisceaux.

Stomates sur les deux faces, enfoncés sous l'épiderme qui fait saillie au-dessus de l'ouverture stomatique. L'épiderme zigzagué laisse voir sur des coupes tangenlielles des stomates élégants, entourés d'épaississement pariétaux en forme de $2 \mathrm{~V}$, qui se font vis-à-vis et qui recouvrent les cellules du stomate (fig. $23 a . b . c$. )

Au centre dans l'espace situé entre les $2 \mathrm{~V}$, la fente de l'appareil se laisse apercevoir. 
Vus, au contraire par dessous, ces stomates présentent la structure habituelle, laquelle se trouve ici compliquée par des épaississements locaux el la proéminence des cellules épidermiques voisines $(c$.)

Tige à épiderme dont les cellules proéminent en saillies rondes et parfois aussi en crochets pointus, de forme triangulaire, à angles aigus présentant sous l'épiderme de chaque arête un groupe d'éléments mécaniques.

Faisceaux en grand nombre, sur deux rangs, dont les marginaux sont portés sur des colonnes allongées et s'anastomosent souvent fréquemment entre eux.

Cellules tannifères nombreuses autour des colonnes de stéréome, des lacunes et des faisceaux.

Stomates dans les types jeunes presque superficiels ou paraissant quelque peu enfoncés par suite de l'élévation des deux cellules voisines au-dessus du niveau des autres.

Rhizone traçant, assez puissant, présentant dans son parenclyyme cortical des lacunes séparées les unes des autres par des rayons formés d'une rangée de cellules parenchymateuses rondes, rayons qui portent généralement des faisceaux libéro-ligneux collatéraux.

Passant au cylindre central, nous trouvons ici aussi pour le tissu conducteur la structure collatérale. Chaque faisceau est entouré d'une très forte gaine de fibres.

Chez plusieurs de ces faisceaux, au lieu de trouver deux grandes cavités correspondant aux gros vaisseaux, nous n'en voyons qu'une seule. Zone péricambiale très accentuée.

Cellules de l'endoderme à parois non épaissies en U, mais d'égale force sur tout leur contour (fig. 149:) A la périphérie, anneau sclérenchymateux composé de cellules hexagonales disposées sur six ou huit couches concentriques. 


\section{5}

Les nodosités dans les colonnes du parenchyme coltical différent de celles qui se trouvent dans les racines par leur aspect plus ou moins pyriforme. Elles proviennent aussi soit d'un collapsus soit d'un plissement survenu dans les cloisons sous l'influence des pressions extérieures, soit encore du collement d'un fragment de cloison résorbée contre une autre cloison qui a persisté (fig. 150).

Racine dont le péricambium se trouve interrompu entre les vaisseaux primaires et l'endoderme. Celui-ci est formé de grandes cellules, épaissies en $\mathrm{U}$ et ponctuées, a lamelles moyennes lignifiées (fig. 151).

Extérieurement à l'endoderme, le tissu se compose de cellules régulières, de forme ovale, laissant entre elles des méats el allant en augmentant de diamètre du centre à la périphérie, comme dans le type normal. Bientôt ce tissu devient parenchyme à cloisons minces et noueuses.

A la périphérie même anneau sclérenchymateux formé des mêmes éléments hexagonaux.

Carex Paludosa. Good. - Reich. Icon. VIII, 644.

Fenille à ćpiderme lisse sur la page supérieure et dont les cellules à parois externes fortes sont pourvues de grands lumens. Sur la page inférieure, nous trouvons au contraire un grand développement de papilles en houcles. Les cellules de plissement forment ici un complexus dont les éléments revêtent la forme collenchymateuse et dont ceux de la série externe diffẻrent fort peu des autres cellules épidermiques.

Faisceaux libéro-ligneux munis de revêtements fibreux très puissants, surtout vis-à-vis des nervures principales ; ces revètements ne forment pas une colonne continue, mais présentent dans leur longueur des solutions de 
continuité ou simplement des étranglements à partir desquels ils s'étalent en éventail d'un côté vers le faisceau et de l'autre vers l'épiderme. Ils sont accompagnés en outre de cellules aqueuses qui se prolongent le long des colonnes.

Pas de cellules tannifères dans le parenchyme assimilateur. Stomates de structure ordinaire sur la face inférieure seulement, en général entourés de deux papilles, qui les font paraître enloncés dans les tissus.

Examiné en coupe tangentielle, l'épiderme de la page inférieure se montre composé de cellules à parois zigzaguées renfermant chacune un cercle à contours épais, lequel correspond à une papille.

Tige triangulaire à angles très aigus fortifiés par un groupe sous-épidermique de fibres mécaniques.

Faisceaux libéro-ligneux en grand nombre (plus de 45), presques tous marginaux, munis de forts revêtements qui font quelque peu saillie en dehors.

Système lacunaire très développé de sorte que les faisceaux jouent aussi le rôle de colonnes de soutien, divisant ainsi le tissu parenchymaleux en une série de grandes lacunes, autour desquelles se rassemble le tissu ver't très réduit.

Stomates de structure analogue à ceux de la feuille, enfermés dans un épiderme zigzagué.

Dans leur course, les faisceaux ne restent pas en nonbre constant: en parcourant la tige florifère, ils subissent des bifurcations, de sorte que, dans les parties supéricures, les sections transversales en font voir un nombre beaucoup plus grand que les autres parties. Outre des anastomoses fréquentes, on peut observer encore des passages de la forme collatérale à la forme concentrique.

Au delà du nœud floral, tous les faisceaux reprennent 
leur place primitive et réapparaissent en plus petit nombre.

Rhizome à faisceaux concentriques présentant à la périphérie du cylindre central quelques formes de transition à la structure collatérale, à phloëme composé de grands tubes criblés et de cellules annexes petites. Dans le tissu cortical, petites lacunes serrées les unes contre les autres et allongées radialement.

Endoderme épaissi en U et cela très fortement.

Sous l'épidcrme court un anneau sclérenchymateux formé en général de deux ou trois assises de cellules fortes et interrompu fréquemment par le parenchyme cortical.

Racine de structure très puissante, pourvue à sa périphérie d'un très fort anneau sclérenchymateux, présentant un endoderme extraordinairement épaissi en $U$ et un parenchyme cortical analogue comme distribution à celui de C. maxima (voir fig. $114 \mathrm{~A}$.)

Péricambium interrompu le plus souvent par les vaisseaux primaires. Lames vasculaires très courtes; faisceaux libériens composés de tubes criblés très grros au nombre de quatre on cinq et accompagnés souvent d'autres éléments plus petits.

\section{Ampullacea. Good. (Reich. Icon. VIII, 659.)}

Feuille à épiderme papilleux, mais d'une façon plus marquée sur la face supérieure (fig. $153 a b$.)

Cellules de plissement, grandes, sur un rang, étalées en ćrentail, brusquement différenciées des autres cellules épidermiques.

Les éléments des deux épidermes diffèrent fort peu quanı à leur grandeur et à leur forme.

Disposition des faisceaux comme chez C. riparia; il en 
est de même pour les cellules aqueuses, les colonnes de sclérenchyme, les lacunes, etc.

Stomates comme chez C. riparia, enfoncés sous l'épiderme, nombreux sur la face supérieure, paraissant moins enfoncés sur la face inférieurc. On peut d'ailleurs trouver plusieurs formes de transition, mais toutes montrent un enfoncement plus ou moins prononcé, qui paraît provenir de la grandeur variable des papilles adjacentes.

Vus de face, ces organes se présentent sous une forme des plus élégantes, dont le type se rapproche de celui de C. riparia (fig. 35 a.b.c.)

Tige triangulaire, crénelée, richement pourvue d'éléments mécaniques qui, d'abordl réduits à servir de revêtements fibreux et de gaines protectrices aux faisceaux, vont en s'étalant sous l'épiderme à mesure que l'on monte vers les épillets.

Faisceaux arrangés d'après le type ordinaire sur deux rangs, pourvus de gaines épaissies d'une façon plus considérable autour des phloëmes.

Epiderme à parois extérieures massives, portant aussi des stomates enfoncés, dont les éléments se bossifient sur les côtés et au-dessus des cellules de fermeture, de manière à donner la même figure que dans la feuille, avec cette différence toutefois qu'on ne voit plus apparaittre de papille sous forme de cercle, comme cela a lieu dans cette dernière.

Cet organe présente, malgré.ses contours plus arrondis et ses ailes moins effilées, une grande analogie avec la tige de C. riparia.

Rhizome traçant, muni à sa périphérie de cinq à six couches environ d'éléments sclerenchymateux à lamelles moyennes fortement lignifiées. A cette zone protectrice succèdent les ccllules à parois minces du parenchyme, disposées en colonnes et laissant entre elles de grandes lacunes allongées radialement. 
Au devant du corps central, se trouvent plusieurs couches concentriques de ces mêmes cellules, qui laissent entre elles de petits méats.

L'endoderme est constilué par des éléments arrondis, épaissis en croissant et dont les parois sont de forme ordinaire moyenne (fig. 154 b.)

Faisceaux à l'intérieur du cylindre central tous nettement collatéraux avec phloëmes normalement constitués.

Gaines fibreuses formées de sclérenchyme, dont les éléments disposés, au maximum en deux assises, sont pourvus de grands lumens et de parois fortes.

Nombreuses anastomoses de ces gaines entre elles. Faisceaux dans les colonnes du parenchyme cortical, lequel présente les mêmes nodosités que le tissu correspondant chez C. riparia. Celles-ci, plus faciles à observer que chez l'espèce ci-dessus (fig. $154 a$ ) proviennent de plissements dans les membranes, plissements qui, suivant leur plus ou moins grande profondeur, donnent par suite du collapsus des corps noueux d'aspects divers.

Nous avons pu jusqu'ici constater la plus grande ressemblance entre les diverses parties du rhizome des deux espèces, C. riparia et C. Ampullacea. Ce sera l'endoderme qui maintenant va nous fournir un caractère distinctif.

En effet, celui-ci est formé d'éléments qui ont une tout autre forme que celle observée chez l'espèce qui précède ; chez l'une, nous arons la forme en croissant, avec épaississement interne; chez l'autre c'est la forme en U qui prévaut avec une épaisseur des parois égale à peu près sur tout le pourtour des élérnents (voir fig. 151) (fig. 156.)

Racine dépourvue de péricambium entre les vaisṣeaux primaires et l'endoderme, lequel est composé d'éléments massifs épaissis en $\mathrm{U}$.

Anneau sclérenchymateux périphérique très forı, comme du reste celui des espèces précédentes. 
Parenchyme cortical présentant la structure habituelle (C. maxima, fig. 114 A) et dont les cloisons, au voisinage de l'endoderme, laissent voir des bifurcations nombreuses, faciles à examiner sur des sections longitudinales (fig. 155.)

Carex vesicaria L. (Reich. Icon. VIII, pl. cClxxvi.)

Feuille à épiderme de structure ordinaire, à cellules plus grandcs sur la page supérieure ; lisse, complètement dépourvu sur les deux faces de poils ou de papilles.

Faisceaux libéro-ligneux très rapprochés de l'épiderme inférieur, portés sur des colonnes composées par le faisceau lui-même, son stéréome et les cellules aqueuses qui sont ici considérablement développées.

Grandes lacunes quadrangulaires contre les bords desquelles se serre un tissu vert assez réduit.

Cellules de plissement très grandes, non brusquement différenciẻes des autres cellules de l'épiderme, comprimées en éventail, sur plusieurs rangs et à jointures peu ou pas collenchymateuses (fig. J.57.)

Colonnes de fibres mécaniques très étroites, mais très allongées, souvent discontinues.

Pas de cellules tannifères.

Stomates superficiels sur les deux faces, de même structure que dans l'espèce C. psendo-Cyperrus et dans un même épiderme zig̨zagué.

Tige triangulaire, à angles aigus et à surface dépourvue de poils ou de papilles, présentant des faisceaux libéroligneux sur deux rangs et un développement considérable d'éléments mécaniques.

En effet, au lieu d'avoir ici des colonnes de ces éléments formant revêtement à l'extrémité externe des faisceaux el un deuxième groupe de ces mèmes cellules, protégeant la pointe intérieure, comme c'est le cas ordinaire, nous 
voyons ici les revètements à l'extrémité interne s'allonger en colonnes, surtout chez les petits faisceaux, les grands conservant pour la plupart l'aspect habituel.

Ces colonnes se continuent dans le parenchyme en formant une série de groupes fibreux interrompus ou continus, lesquels ront rejoindre sourent la gaine fibreuse des grands faisceaux en formant avec elle une sorte de pont.

Lacunes nombreuses séparées par les faisceaux et leurs colonnes.

Epiderme à parois épaisses portant les mêmes stomates superficiels. Parenchyme assimilant richement pourvu de bifurcations et de cellules en entonnoir.

Rhizome longuement traçant, à faisceaux concentriques, (dont les phloëınes présentent l'aspect normal) entourés, d'une forte gaine d'éléments mécaniques (fig. 158.)

Cellules endodermiques ayant mème forme que celles de la racine dans C. pseudo-Cyperus, à lamelles moyennes lignifièes.

Sous l'épiderme se trouve un anneau d'éléments sclérenchymateux de forme polygonale, à parois épaissies et ponctuées, disposés sur cinq ou six assises; sous l'endoderme nous avons une couche de cellules péricambiales sclérifiées.

Nombreux méats et hifurcations dans le parenchyme corlical ; ces dernières se retrouvent aussi dans le parenchyme ligneux du cylindre central.

Racine dépourrue de péricambium entre les vaisseaux primaires et l'endoderme, présentant à la périphérie un anneau de sclérenchyme formé de quatre à cinq couches de cellules polygonales à parois épaisses.

L'assise la plus externe du tissu cortical conserve quelques prolongements papilleux. Cellules endodermiques de mème forme que chez C. pseudo-Cyperus. Nême analogie 
172

quant à l'arrangement des tissus ; mêmes nodosités, méats et ponctuations.

Les cinq espèces que nous venons d'étudier, savoir :

C. pseudo-Cyperus

C. riparia

C. paludosa

C. ampullacea

C. vesicaria

forment entre elles un groupe dont les membres présentent de grandes ressemblances, soit sous le point de vue systématique, soit aussi sous celui de la structure anatomique.

L'étude de leurs tissus peut cependant nous permettre de les différencier facilement, comme le montre le tableau suivant, dans lequel sont données seulement les différences les plus importantes : 


\begin{tabular}{|c|c|c|c|c|c|}
\hline $\begin{array}{c}\text { Carex } \\
\text { vesicaria }\end{array}$ & $\begin{array}{c}\text { Carex } \\
\text { ampullacea }\end{array}$ & $\begin{array}{c}\text { Carex } \\
\text { Paludosa }\end{array}$ & $\begin{array}{c}\text { Carex } \\
\text { Riparia }\end{array}$ & $\begin{array}{c}\text { Carex } \\
\text { P. Cyperus }\end{array}$ & 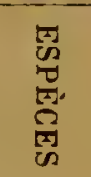 \\
\hline 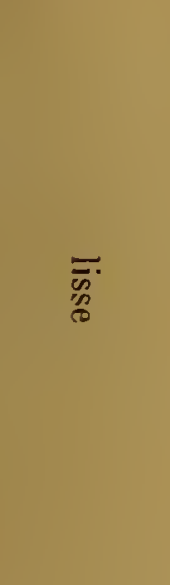 & 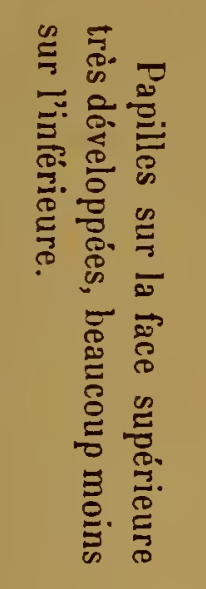 & 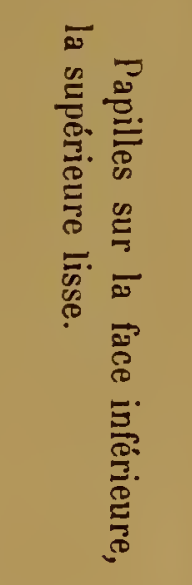 & 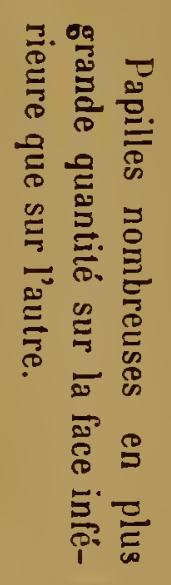 & 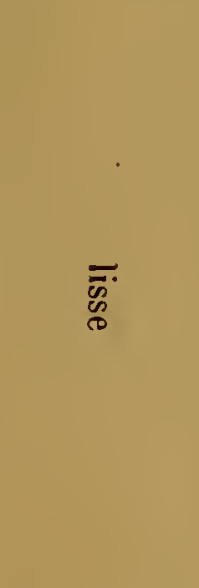 & 荘 \\
\hline 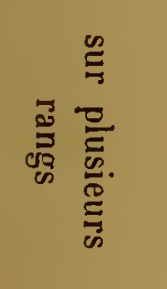 & 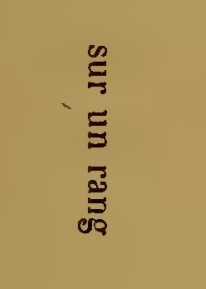 & 咅 & 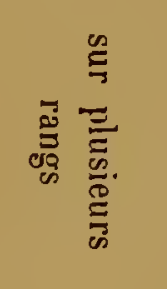 & 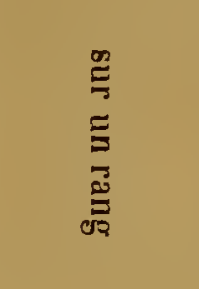 & 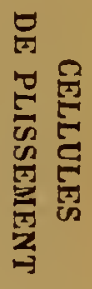 \\
\hline 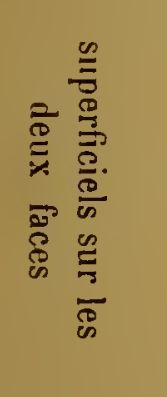 & 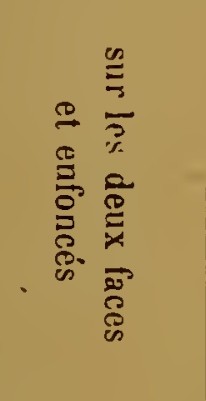 & 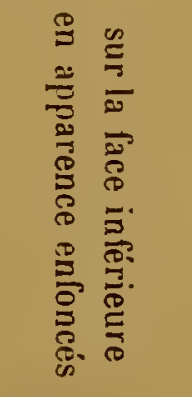 & 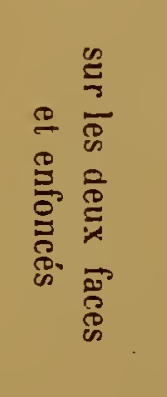 & 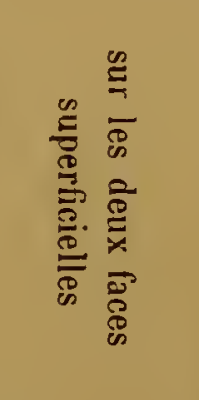 & 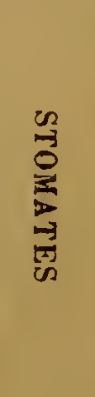 \\
\hline 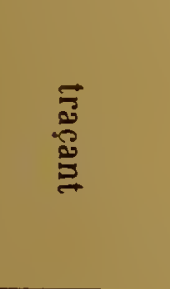 & 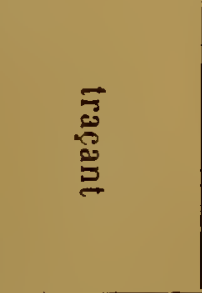 & 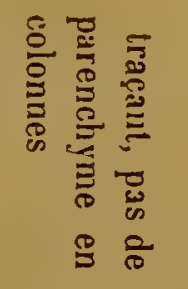 & 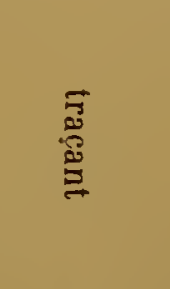 & 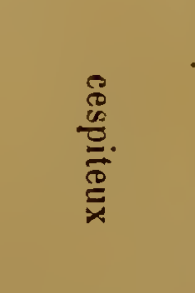 & 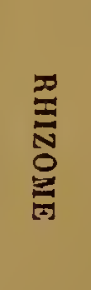 \\
\hline 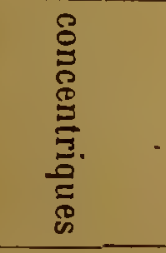 & 总 & 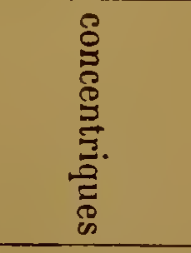 & 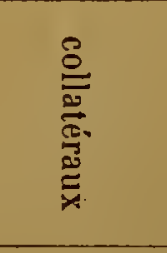 & 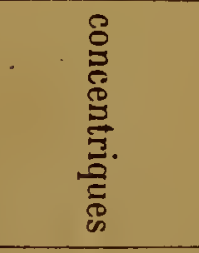 & 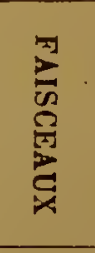 \\
\hline 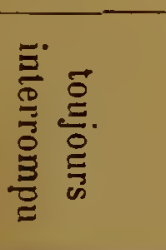 & 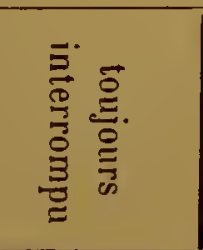 & 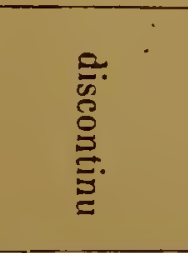 & 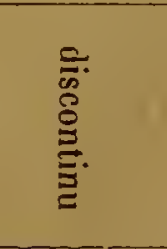 & 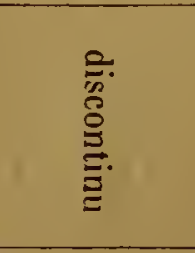 & 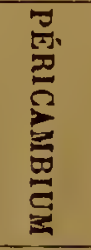 \\
\hline
\end{tabular}


Carex hirta L (Reich. Icon. VIII, 628.)

Feuille à épiderme poilu, composé sur la page supérieure de cellules arrondies, de grandeur à peu près égales, présentant des cellules de plissement disposées sur deux rangs à jointures collenchymateuses et dont le rang extérieur est formé d'éléments peu et graduellement différeneiés.

Sur les arêtes, quelques papilles développées.

Sur la face inférieure, nous avons, formant l'épiderme, des éléments plus petits, à parois externes lisses, renfermant les stomates.

Faisceaux marginaux entourés d'une gaine fasciculaire de force moyenne et d'un système de cellules aqueuses très développé, longeant leurs flanes et formant une colonne à leur extrémité supérieure jusque sous les cellules de l'épiderme (fig. 168.)

Revêtements fibreux relativement faibles, plus développés à la pointe inférieure des faisceaux, très peu à l'extrémité supérieure. Chez les plus grands de ees derniers, nous avons une colonne de cellules fibreuses allant jusqu'à l'épiderme, mais le plus souvent ces éléments mécaniques se bornent à former de petits groupes sous-épidermiques en regard des faisceaux principaux (fig. 160.)

Pas de cellules tannifères.

Les poils qui se rencontrent dans cette espèce présentent des parois plus ou moins noueuses; ils sont unicellulaires, offrant à leur extrémité une pointe épaissie; on les trouve indifféremment sur les deux pages de la feuille, mais de préférence sur la supérieure (fig. 161.)

Stomates superficiels répondant au type ordinaire de structure, pourvus de grandes chambres respiratoires. (fig. $17 a$ et $b$. ) 
Tige triangulaire creuse, présentant pour ses faisceaux libéro-ligneux la disposition habituelle sur deux rangs, à épiderme lisse portant des stomates superficiels de même espèce que ceux de la feuille.

Faisceaux offrant une structure de force moyenne, pourvus de gaines fasciculaires dont les éléments présentent des parois plus minces que dans les cas habituels.

Rhizome traçant montrant à la périphérie plusieurs couches de cellules à cloisons tangentielles, recouvrant un anneau sclérenchymateux formé de cellules polygonales dont les parois sont moyennement épaissies.

Dans le cylindre central, nous avons des faisceaux libéroligneux disposés sans aucun ordre apparent, à phloëmes normaux, et s'anastomosant par leur's gaines fibreuses au voisinage de l'endoderme. Ces faisceaux dans cette partie du cylindre central sont en majeure partie concentriques, présentant ici et là une forme intermédiaire et devenant nettement collatéraux au milieu de la tige. On y observe en même temps une diminution des grros vaisseaux qui souvent sont réduits à l'unité dans un même faisceau.

L'endoderme du cylindre central est composé d'éléments en U, épaissis sur tout le pourtour, mais d'une quantité double environ sur les parois internes.

Le tissu parenchymateux très lâche, riche en amidon et présentant dans son intérieur de petites lacunes, montre en outre de nombreux méats intercellulaires.

Racine velue, construite d'après le type ordinaire, présentant sous l'épiderme un anneau de sclérenchyme qui se change plus à l'intérieur en tissu à cellules rondes, encore assez fortes et tombant en collapsus dans les vieilles racines; chez les jeunes, tout le parenchyme cortical, situé entre l'endoderme et le sclérenchyme de la périphérie, est formé d'éléments ronds, allant croissant en diamètre du centre au pourtour de la racine. Plus tard 
tout ce tissu se résorbe, laissant ici et lả comme pont, une des séries radiales et deux ou trois assises servant d'enveloppes à l'endoderme.

Celui-ci est formé d'éléments comprimés entre eux radialement et épaissis jusqu'à la moitié environ de leur longueur totale. Ils sont en contact immédiat avec la plupart des vaisseaux primaires, lesquels font partie de lames vasculaires Irès courtes.

Péricambium, - là où il existe - formé de cellules à parois épaisses et lignifiées

Les espèces que nous venons de passer en revue sont de celles que nous rencontrons dans nos contrées; sous ce rapport-là, elles méritent plus spécialement notre atten-

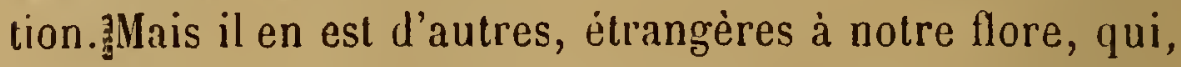
à plus d'un point de vue, ne sont pas moins dignes d'intérêt.

Celles dont l'ètude va suivre sont de ce nombre. Elles vont nous montrer en effet d'une façon éloquente l'influence que peuvent avoir sur la structure anatomique des conditions de végétation différentes.

Ces espèces sont les suivantes:

1. Carex arenaria, qui est très répandue dans les contrées septentrionales de l'Europe, végétant dans les terrains légers, sablonneux qui offrent par conséquent peu de résistance à la croissance.

2. Carex provincialis, plante robuste, habitant les marais du midi de la France. (Appartient au groupe de C. præcox, Jacq.)

3. Carex Grayi Carey, qui a pour patrie les fleuves et les rivières de l'Amérique du nord (Illinois, Ohio ${ }^{1}$.)

4. C. baldensis. Espèce qui végète dans les lieux arides

1 Gray's Manual of Botany, p. 598. 


\section{7}

et pierreux de l'Italie septentrionale et appartient au groupe de G cyperoïdes L.

5. C. hordeistichos. Vill. qui appartient au groupe de C. hirta et qui a pour patrie les prairies sablonneuses et marécageuses de l'Allemagne el du nord-est de la France.

C. arennrin. L. (Reich. Icon. VIII. 55l)

(Espèce voisine de C. disticha. Huds. et de C. leporina, L.)

Feuille dont l'épiderme est assez fortement constitué et notamment sur la page supérieure où la paroi externe des cellules se termine souvent en papille nettement accusée, de forme pyramidale, tandis que sur la page inférieure on n'observe que peu ou pas d'aspérités.

Disposition générale des faisceaux libéro-ligneux conme chez C. hirta, mais ici les colonnes de sclérenchyme traversent toute l'épaisseur de la feuille.

Ces faisceaux présentent une gaine fasciculaire assez forte bordée sur ses côtés d'un rang de cellules aqueuses, lesquelles se prolongent quelquefois au delà de l'extrémitẻ du faisceau ver's l'épiderme.

Cellules de plissement très allongées dans le sens radial et comprimées entre elles, disposées sur un seul rang qui fait contraste brusque avec les autres éléments de l'épiderme.

Le contour de la feuille est légèrement ondulé, présentant dans chaque enfoncement une ou deux cellules épidermiques plus grosses que les autres sur la face supérieure el vis-à-vis de chaque faisceau.

Cellules à cônes siliceux sur la face inférieure, moins visibles sur l'autre.

Lacunes arrondies, dans un parenchyme dépourvu de cellules tannifères.

Stomates grands, à niveau de l'épiderme, présentant de grandes chambres respirạtoires, un lumen très allongé 


\section{8}

et étroit, ce qui indique un fort épaississement des cellules stomatiques (colorces en bleu intense par le vert d'iode), visibles seulement sur la page inféricure, et présentant, vus de face, le même aspect que ceux de C. arida, les gouttelettes d'huile en moins (fig. 28 et 31.) Tout l'appareil stomatique est lui-même quelque peu plus grand et plus allongé.

Tige triangulaire à angles arrondis pourvue d'un épiderme lisse ì parois extérieures fortes, formé de cellules régulières portant des stomates superficiels au fond d'une très légère dépression et des faisceaux disposés sur un rang, munis - les plus gros - de revêtements fibreux longs et larges allant jusqu'à l'épiderme.

Cellules d'angle terminées le plus souvent en papillescrochets. Gaines fasciculaires présentant un épaississement plus considérable au pourtour du liber des faisceaux.

Nombreuses bifurcations dans les membranes des cellules du parenchyme assimilant, orientées toutes en général dans le sens du rayon.

Rhizome faisant contraste par l'aspect peu résistant que présente sa structure; on y remarque en effet un anneau de sclérenchyme à la périphérie, mais celui-ci est à peine plus accentué dans ses parois que les éléments du parenchyme cortical moyen; il n'est reconnaissable qu'à la forme polygonale des cellules qui le composent.

Celte écorce moyenne se trouve constituée par des cellules rondes à parois minces formant colonnes ou rayons et qui séparent entre clles de grandes lacunes, nombreuses et quadrangulaires (fig. 186).

D'autre part les cellules de l'endoderme sont à peine épaissies sur leur côté interne. Les faisceaux libéro-ligneux netternent concentriques pour la plupart - avec quelques lormes intermédiaires au voisinage de l'endoderme - 


\section{9}

sont répartis sur trois ou quatre rangs et sont entourés de gaines fibreuses très épaisses (fig. 114 B.)

Ces gaines, surtout celles des faisceaux situés à la périphérie du cylindre central s'anastomosent entre elles tandis que celles des faisceaux centraux restent séparées les unes des autres par du parenchyme ligneux formé d'éléments à parois minces.

Sous l'endoderme court une zone de cellules analogues d'aspect à celles des gaines fibreuses des faisceaux, et formant péricambium.

Tout le tissu contigu extérieurement à l'endoderme est richement ponctué et pourvu de méats intercellulaires.

Racine normale monocotylédonienne ayant un péricambium non interrompu par les vaisseaux primaires (fig. 162.)

Eléments endodermiques allongés radialement, quelque peu comprimés les uns par les autres, dont les parois sont d'égale épaisseur sur tout leur contour et ont conservé la forme prosenchymateuse primitive. Leur inté. rieur est souvent rempli d'amidon.

Le tissu cortical est formé par des files radiales de cellules parenchymateuses qui, dans la partie moyenne, se résorbent en formant de grandes lacunes, analogues à celles du rhizome.

Anneau srlérenchymateus de force moyenne à la périphérie.

\section{Carex Grayi. Carey.}

(Espèce américaine du groupe des " Lapulinae ${ }^{1}$ " voisine des C. pseudo-Cyperus C.)

Disposition générale de la feuille comme chez C. hirta. Cellules épidermiques arrondies, régulières, à parois extérieures lisses, ne portant des papilles-crochets que sur les arêtes.

\footnotetext{
1 Gray's Manual of Botany.
} 
Cellules de plissement grandes sur un rang, comprimées, étalées en éventail.

Faisceaux libéro-ligneux, richement pourvus de cellules aqueuses soit sur leurs côtés, soit formant colonnes à leur extrémité supérieure. Lacunes grandes et allongées.

Stomates à niveau de l'épiderme à lumens allongés, semblables à ceux de C. hirta, situés exclusivenent sur la page inférieure et ne renfermant apparemment aucune trace de corps huileux.

Tige triangulaire à épiderme très fort et à arêtes scabres dans les parties supérieures et à angles arrondis dans le bas (fig. 183). Cet épiderme est pourvu de stomates superficiels de même forme que ceux de la feuille et en nombre réduit, ses éléments s'appuyant, presque sur tout le pourtour, sur un anneau d'autres éléments sclérenclyymateux. Ce dernier est formé par une superposition de six couches - et quelquefois plus - de cellules à parois trés fortes. Presque continu dans le bas de la tige et relié aux faisceaux par leurs gaines fibreuses, il est dans les parties supérieures fréquemment interrompu par le parenchyme (fig. 182).

A mesure que l'on remonte vers l'épi, on le voit se fractionner de plus en plus et être remplacé par les revêtements fibreux des faisceaux qui acquièrent alors une épaisseur considérable, formant de véritables colonnes de soutien séparées les unes des autres par les lacunes.

Dans le voisinage de l'épi, les faisceaux se rapprochant du centre, anastomosent leurs gaines de telle sorte que sur les sections transversales, on obtient deux zones concentriques d'éléments mécaniques dont l'une, l'extérieure, plus épaisse est reliée aux éléments fibreux sous-épidermiques par des rameaux formés d'èléments senıblables passant entre les lacunes (fig. 182.)

Rhizome de structure habituelle, solidement bâti, pré- 


\section{1}

sentant à la périphérie un épais anneau de sclérenchyme et un endoderme massif s'appuyant sur une zone péricam. biale formée d'éléments lignifiés et à parois résistantes. Les cellules qui composent cet endoderme sont ovales et montrent sur tout leur pourtour une épaisseur à peu près égale (fig. 184.)

Faisceaux libéro-ligneux purement collatéraux entourés de très fortes gaines fibreuses, à phıloëmes normaux et distribués à la périphérie sur deux ou trois rangs, laissant libre le milieu du cylindre central (fig. 184).

Parenchyme dépourvu de lacunes présentant dans ses éléments de nombreuses ponctuations.

Racine dont les poils per'sistent et dont les vaisseaux confinent le plus souvent à l'endoderme sans interposition de péricambium.

Cet endoderme est lui-mème composé de cellules dont les parois internes sont épaissies en $\mathrm{U}$ et les externes sont restées relativement minces; leur forme est quelquefois très allongée.

Sous l'assise pilifère, se trouve un anneau de sclérenchỵme formant un collier puissant lignifié dans sa moitié externe.

\section{Carex baldengis. L}

Feuille remarquable par l'épaisseur des deux moitiés du limbe, pourvue sur les deux faces d'un épiderme lisse formé de cellules régulières, un peu moins grandes sur l’inférieure (fig. 163.)

Cellules de plissement à parois assez fortes sur deux rangs, l'extérieur compose de six à sept cellules très grandes, l’intérieur complant à peu près un nombre égal de petits éléments.

Faisceaux libéro-ligneux occupant le centre de l'épaisseur de la feuille, protégés par une gaine fasciculaire 
dont les éléments très épaissis, le sont encore à un degré plus élevé au pourtour des phloëmes, et par des revêtements allongés en colonnes aux deux extrémités jusque sous l'épiderme.

Chacun d'eux est accompagné sur ses côtés d'une rangée de cellules aqueuses.

Bords marginaux de la feuille présentant des cellules plus grandes, parfois allongėes en crochet acéré.

Tout le tissu chlorophyllien qui avoisine l'épiderme inférieur se montre pourvu de grands méats pariétaux intercellulaires (fig. 49.)

Stomates sur la face inférieure, superficiels, assez gros.

Tige triangulaire creuse dans le bas, à angles arrondis, présentant un épiderme lisse à parois externes épaisses et un arrangement des faisceaux sur deux rangs.

Ceux-ci sont pourrus de revêtements très développés et d'une gaaine dont les éléments offrent sur chacun des côtés et autour du phloëme un épaississement plus considérable de leurs parois.

Stomates superficiels, analogues comme structure à ceux de la feuille, mais moins arrondis et plus allongés (fig. 27.)

Rhizome très solidement construit présentant un épiderme dont les éléments très épaissis renferment ici et là quelques cônes siliceux (fig. 165.)

Le tissu sous-jacent est formé de cellules à cloisons épaissies, tantôt uniformément sur toute leur lonģueur, tantôt seulement aux jointures, de façon à produire un collencliyme bien caractérisé (fig. 165.)

Cylindre central séparé du tissu cortical par un endoderme massif, dout les éléments plus ou moins comprimés prennent des formes diverses (fig. 164.)

Tous les faisceaux libéro-ligneux dispersés au centre comme à la périphérie du cylindre central sont nettement 


\section{3}

concentriques et montrent des phloëmes formés de cellules à peu près d'égale grandeur.

Racine offrant aussi une struclure de résistance très prononcée : nous arons en effet un épais anneau de sclérenchyme de six à sept couches dont les éléments sont lignifiés dans la moitié extérieure.

Tissu parenchymateux se résorbant en lacunes, à l'exception de quelques couches au pourtour de l'endodernie.

Endoderme formé d'éléments réguliers à parois épaisses et s'appuyant directement aux vaisseaux primaires sans interposition de péricambium (fig. 166.)

Carex provineialis. Desgl.

(C. hispilla. Willd.)

Epiderme de la feuille inégalement construit, présentant sur la face supérieure de grandes cellules à parois externes très épaisses.

Sur les bords de la feuille, l'épaississement des parois va en augmentant, n'intéressant plus seulement les parois externes, mais encore les cloisons radiales (fig. $169 d$.) Sur la face inféricure, nous trouvons des éléments épidermiques plus petits, mais tout aussi résistants, prolongés souvent en papilles en forme de boucles.

Quelquefois, sur la face supérieure, on observe qu'une ou plusicurs des cellules épidermiques se développe aussi en papille plus ou inoins pointue ; ces corps sont en général pourvus de parois massives surtoul à leur extrémité (fig. 169 a. b.)

Cellules de plissement disposées sur plusieurs rangs, le plus souvent trois, à jointures collenchymateuses.

Faisceaux libéro-ligneux entourés d'une gaine forte, occupant le centre de la feuille et protégés par des colonnes de stéréome, qui les relient à l'épiderme sans solution de continuité. 
Cellules aqueuses très richement représentées, n'existant pas seulement sur les flancs des faisceaux, mais longeant encore les colonnes et se continuant de même sons l'épiderme parfois en plusieurs couches pour aller rejoindre les éléments analogues correspondants du faisceau voisin (fig. 170 ca.)

Lacunes peu développées relativement à la laille de l'organe. Stomates sur la page inférieure profondément enfoncés dans le tissu sous un épaississement des parois des cellules épidermiques voisines (fig. 19 et 20.)

Ces stomates sont petits, difficiles à distinguer et possèdent un lumen très réduit et une chambre respiratoire étroite.

Vus de face, ils apparaissent sous un aspect semblable à celui des mêmes organes chez C. glauca. Ici cependant, les parois ont une autre disposition et une épaisseur beancoup plus considérable; leurs contours en outro sont peu ou pas zigzagués.

Parenchyme assimilant présentant de nombreuses cellules tannifères localisées surtout au voisinage des colonnes de stéréome.

Tige triangulaire à faisccaux disposés sur deux rangs et dont ceux du bord sont portés sur de longues colonnes d'éléments fibreux.

Epiderme à parois externes très épaisses portant les mêmes stomates enfoncés profondément et communiquant avec l'air par un canal étroit.

Gaines fasciculaires dont les cellules s'épaississent considérablement au pourtour du phloëme.

De chaque côté des filisceaux, se trouve une rangéc de cellules aqueuses, et, disséminées dans le parenchyme chlorophyllien, des cellules secrétant du tannin.

Rhizome renfermant dos faisceaux concentriques entourés de gaines fibreuses épaisses. 
Endodermes composés d'éléments à contours arrondis présentant partout la même épaisseur et s'appuyant à l'intérieur sur une zone de cellules ì parois fortes et lignifiées (fig. 171.)

Anneau sclérenchymateux occupant à la périphérie le tiers environ du tissu corlical, leyuel forme des lacunes et renferme une grande quantité d'amidon. Les parties voisines de l'endoderme renferment un grand nombre de ponctuations, de méats et de bifurcations dans les membranes cellulaires. Ces bifurcations se retrouvent aussi dans le parenchyme du cylindre central (fig.172, 173, 174.)

Racine dont le péricambium court d'une façon continue entre l'endoderme el les raisseaux primaires.

Eléments endodermiques de même forme que ceux du rhizome, un peu plus comprimés cependant, arec une tendance à un épaississement des parois internes.

Disposition habituelle du tissu cortical ; nodosités nombreuses el anneau de sclérenchyme à la périphérie.

Carex hordeigtichos. Will. (Reich. Icon, 627.)

Cette espèce prèsente une feuille solidement bâtie dans toutes ses parties. Les épidernes des deux faces s'éıuivalent à peu près comme grandeur; tous deux présentent à l'extérieur une paroi très forte, lisse súr toute la surface et sont munis de cônes siliceux de grande taille (fig. 178, 176.)

Les cellules de plissement disposées sur un rang, presque pas différenciées des autres éléments épidermiques, possèdent des parois plus fortes que dans la plupart des espèces étudiées plus haut; leurs jointures sont en outre collenchymateuses (fig. 175.)

Le mésophylle renferme un grand nombre de méats et de bifurcations très prononcées, orientées toutes dans le sens de l'épaisseur (fig. 179.) 
Pas de cellules tannifères.

Faisceaux libéro-ligneux occupant le milieu de la feuille, entourés des cellules aqueuses disposées sur un rang et flanquées de revêtements très développés. Leur gaine est formée d'éléments à parois internes extraordinairement épaissies, principalement autour du phlö̈me (fig. 177.)

Stomates grands, sur les deux faces, à fleur d'épiderme et dont les cellules annexes avancent profondément dans la chambre slomatique (fig. 22.)

Lacunes grandes, allongées perpendiculairement à la surface de la feuille.

Tige triangulaire dans le bas, à angles très arrondis, présentant un grand nombre de faisceaux disséminés sur plusieurs rangs qui se réduisent à deux dans les parties supérieures, où ces organes sont alors portés sur de larges et fortes colonnes d'éléments fibreux.

Epiderme à parois extérieures lisses et épaisses faisant suite à un anneau sous-épidermique de cellules collenchymateuses.

Stomates superficiels, cônes siliceux d'une grosseur considérable.

Gaines fasciculaires formées de cellules à parois très épaissies autour du phloëme et entourées d'éléments fibreux en triple et quadruple couche.

Au passage à travers le nœud floral, les faisceaux libéroligneux gagnent comme à l'ordinaire le centre de la tige, mais laissent sous l'épiderme leur's revêtements, de sorte qu'on obtient de longues colonnes d'éléments mécaniques qui portent à leur extrémité les fitisceaux ei séparent entre elles les lacunes, en réduisant considérablement le parenchyme assimiláteur.

Rhizome cespiteux, construit tıès solidement, présentant à sa périphérie un épiderme à parois fortes contenant de grands cones siliceux et doublé d'une zone de tissu 
collenchymateux, lequel est à son tour interrompu par des groupes de fibres à parois épaisses et lignifiées.

Ce collenchýme se continue ensuite à l'intérieur en parenchyme à cloisons très accentuées, richement pourvues de méats et de ponctuations.

Faisceaux concentriques à phloëme présentant des cellules sensiblement égales en diamètre et entourés de gaines fasciculaires puissantes.

Endoderme à éléments épaissis en U, très fort, et par endroits double (fig. 180.)

Racine dont les vaisseaux primaires sont tantôt séparé:s de l'endoderme par une assise de péricambium, tantòt sont en rapport direct avec ses éléments. On observe cependant une prédominance marquée de ces derniers.

Anneau de sclérenchyme à la périphérie formé par quatre à cinq couches de cellules lignifiées dont les plus internes ne tardent pas à collapser.

Endoderme très fortement épaissi sur la moitié qui regarde.le cylindre central (fig. 181.)

\section{CONCLUSIONS}

Par l'étude des espèces qui précèdent, nous voyons que si les organes de végétation des Carex répondent tous à un plan qui se retrouve dans chacune d'elles, il existe cependant des différences assez nombreuses el considérables.

Si l'on veut maintenant, se basant sur tel ou tel de ces organes - comme les stomates, les faisceaux du rhizome, etc. - rapprocher entre elles toutes les cspeces, qui ont sous le rapport de ces parties quelques affinités, on ne tardera pas à reconnaître l'impossibilité d'un groupement 
quelconque reposant sur les caractères anatomiques offerts par les organes de végétation de ces espèces. On arriverait en effet, d'un côté à rapprocher les unes des autres des espèces systématiquement éloignées et, vice-versa, à en séparer d'autres très voisines. Si, par exemple, nous nous basons sur les stomates pour opérer un groupement, et si nous cherchons à l'éunir ensemble les espèces qui ont ces organes à la superficie de l'épiderme contre un autre groupe qui serait formé de celles qui ont ces mêmes organes enfoncés dans les lissus, nous verrions figurer côte à côte des espèces très différentes, telles que C. paniculata, C. provincialis, C. glauca, C. ampullacea et bien d'autres; dans le second groupe nous verrions $C$. hirta marcher de pair avec C. alba, C. muricata, et dans un troisième, nous aurions encore réunis ensemble $C$. Buxbaumii et C. stricta.

Appliquant aussi le même procédé aux rhizomes au point de vue de la structure des faisceaux libéro-ligneux, nous aurions, il est vrai, analogie entre les C. riparia et ampullacea (faisceaux collatéraux), mais par contre nous serions obligés d'en séparer C. paludosa, C. pseudoCyperus, C. vesicaria, qui forment sans cela un groupe très intime avec les deux premières espèces nommées cidessus.

$1 l$ résulte donc de ce qui précède que si l'on arrive parfois à constater entre des espèces voisines systématiquement des rapports intimes au point de vue anatomique, ces caractères anatomiques deviennent une confirmation du fait que les espèces en question sont proches parentes. Nous en arons vu chemin faisant quelqués exenples, mais ils ne peuvent pas devenir la base de rapprochements systématiques, puisqu'ils sont le résultat d'adaplations encore inexpliquées.

En effet, considèrant toujours les stomates, comment 
se fait-il que chez des espèces, toutes marécageuses, souvent très voisines (C. riparia, C. vesicaria), nous ayons toujours ces organes différemment situés par rapport à l'épiderme, à savoir enfoncés chez les unes, superficiels chez les autres? Faut-il admettre l'idée dejà émise, que cette variété dans la structure stomatique provient du fait que les conditions de végétation, dans lesquelles la plante a commencé à vivre, ont changé petit à petit, et que de submergé qu'ètait le sol, par exemple, celui-ci, dans la suite est devenu sec et a influé ainsi sur le développement ultérieur de certains organes?

Ce sont là des questions qui demandent à être traitées spécialement et dans le détail desquelles nous ne pourons entrer maintenant '.

Constatons seulement ce fait qu'à la plupart des espèces vivant dans des stations marécageuses correspondent des stomates placés à la superficie de l'épiderme, tandis qu'au contraire, chez les espèces qui sont appelées à vivre dans des terrains dont la nature et la qualité peuvent changer par suite des circonstances atmosphériques ou autres, ces mêmes organes se trouvent enfoncés sous l'épiderme, arrangement qui a sans doute pour but une transpiration moins rapide. De semblables dispositions nous sont fournies par C. gliuca, C. nigra, etc.

Il y a de nombreuses exceptions, mais la grande majorite des Carex étant formée de plantes marécageuses, il n'y a rien d'étonnant, si nous y rencontrons le plus sourent des stomates superficiels.

1 Nous aurions voulu faire entrer'dans le cadre de ce travail, le $e^{s}$ résultats de recherches opérées sur plusieurs Carex de mème espèce' cultivés dans des milieux différents, mais la germinati in tardive et difticile des graines dans des terrains qui ne sont pas ceux auxquels la plante est habituée, et aussi des motifs personnels, m'ont obligé d'y renoncer pour le moment. 
La structure des plantes, avons-nous dit, n'est pas un effet du hasard; elle doil correspondre à des exigences physiques et physiologiques.

Nous en avons chez les plantes, qui nous ont occupé, des exemples frappants.

Si nous considérons en effet deux espèces systématiquement éloignées, telles que C. arenaria el C. provincialis ou l'une quelconque des formes essentiellement aquatiques, nous serons dès l'abord frappés, non par la variabilité de structure que présentent leurs lissus, mais par les degrés différents de développement que montrent leur's èléments mécaniques.

Dans l'une (C. arenaria), l'anneau sclérenchymateux du rhizome se trouve à peine marqué ; dans l'autre, au contraire, il est extraordinairement développé. Passant ensuite à l'endoderme et au cylindre central, nous pourrons faire ici encore la même remarque.

Chez le premier, les éléments endodermiques ont conservé la forme primitive de prosenchyme sans épaissir d'une façon bien marquée leurs parois internes, et cela dans les plus vieux rhizomes. Chez les seconds et chez ceux qui lui ressemblent, l'endoderme revêt un aspect massif sur tout son pourtour ou seulement - mais alor's d'une façon très accentuée - sur les parois internes de ses éléments. Bien souvent encore, ce développement en vue de la consolidation et de la protection des tissus, ne s'arrête pas là. On a en effet beaucoup d'espèces chez lesquelles l'assise interne contiguë à l'endoderme épaissit ses parois et les lignifie de façon à suppléer au rôle physiologique de l'endoderme.

Chez C. arenaria, c'est le cylindre central lui-mème, qui est chargé d'assurer à l'organe la solidité nécessaire. Ses faisceaux sont dans ce but enveloppés de gaines fibreuses épaisses, lesquelles, par leurs anastomoses réciproques à 


\section{1}

la périphérie du cylindre, donnent au rhizome un degré de résistance plus considérable.

Dans les espèces aquatiques, ce rôle de renforcement des tissus mécaniques est encore plus complet, puisqu'à l'action des faisceaux plus ou moins anastomosés, vient s'ajouter l'effet protecteur d'un endoderme puissant, lequel est lui-même protégé par un anneau sclérenchymateux périphérique très développé.

Il ressort donc de ces considérations que, dans les parties souter'aines à l'abri de l'action des courants de l'air et de l'eau, les tissus présenteront une structure de résistance centrale et que cette structure offrira un degré de développement plus élevé chez les espèces qui végètent dans un milieu plus résistant.

La différence est aussi marquée entre C. arenaria et tout autre espèce ayant pour station un terrain à la fois sec, aride el pierreux. On en aura la preuve, si l'on compare la structure si différente que présente l'espèce ci-dessus avec celle des C. baldensis, nitida, humilis, ornithopoda, lesquels habitent des stations très différentes. De mème nous aurons des degrés intermédiaires, clans les individus qui ont pour habitat les bois et les forèts. Là encore, nous avons une tendance à une distribution mécanique centralę, mais différant quelque peu quant au développement en vue de la résistance, de celle des espèces marécageuses. Nous n'y trouvons pas, comme chez ces dernières, des organes qui soient capables de résister au poids d'une quantité considérable d'eau.

Chez la racine, les différences de structure sont encore plus marquées. C'est à peine, en effet, si chez C. arenária, on trouve des éléments mécaniques sous forme d'anneau sclérenchymateux à la périphérie. Comme dans les racines en général, nous avons bien des éléments de forme prosenchymateuse, différảnt par leur aspect des cellules rondes 
du parenchyme, mais leur lorce reste égale sous le rapport de l'épaisseur des cloisons.

L'endoderme, plus encore que dans le rhizome, a conservé son caractère primitif, et n'étail sa forme spéciale, on ne le distinguerait en rien des éléments du parenchyme.

Tout autres sont les racines des espèces aquatiques qui présentent un anneau périphérique de sclérenchyme, cn général très fort, el un endoderme à parois massives, le plus souvent encore entouré à l'extérieur par trois ou quatre couches de cellules parenchymateuses épaissies. Ce que nous avons dit des rhizomes pourra donc également s'appliquer aux racines.

Si l'organe doit être susceptible de résister aux forces qui agissent sur lui pour le plier, les éléments mécaniques devront être arrangés à la périphérie. La preuve nous en est fournie par les tiges et les feuilles.

Ces organes nous présentent, en effet, des éléments mécaniques toujours périphériques. La raison de cet arrangement est facile à saisir, quand on songe au rôle qu'ils ont à remplir, rôle qui consiste à résister aux vents et à supporter le poids des organes floraux terminaux, lequel poids chez les plantes qui nous occupent peut dęvenir considérable.

Il reste, en terminant, une dernière remarque à faire à propos de l'endoderme, soit dans les racines, soit dans les rhizomes.

Nous avons pu voir, en étudiant les quelques espèces détaillées plus haut, qu'à part de très rares exceptions, l'endoderme des deux organes est en général considérablement épaissi.

On peut se demander à quelle cause se rattache cet épaississement. A-t-on à faire ici seulement à une disposition protectrice, ou bien faut-il y voir encore un rapport direct entre l'habitat de l'espèce et l'espèce elle-même ? 


\section{3}

On sait en effet que l'endoderme, qui est gènéralement subérifié, a pour fonction d'assurer l'imperméabilité des tissus. Or, chez les Carex, l'observation vient confirner cette manière de voir, car nous avons pu constater que c'est justement chez les espèces vivant dans des stations marécageuses que cette assise se développe de la façon la plus marquée, el que chez d'autres espèces, qui végètent dans des terrains arides, cet endoderme se trouve à peine différencié des autres couches d'éléments (C. arenaria.)

Quant à nous, notre avis sur la matière, est que nous avons à faire ici aux deux fonctions réunies, à savoir d'un còté, protection du cylindre central, et de l'autre imperméabilisation des tissus. 


\section{THESES}

I

On ne peut en aucun cas se baser sur des caractères anatomiques pour grouper systématiquement les espèces dans le genre Carex.

II

Les Cypéracées - et en particulier les Carex - renferment aussi des cellules sécrétrices.

III

Le produit de cette sécrétion est du tannin.

IV

A un habitat dans un terrain léger, sec et peu résistant correspond une structure mécanique centrale, très peu accentuée.

Un terrain submergé ou sec el pierreux, exige au contraire un développement mécanique central non seulement plus puissant, mais encore renforcé par un sclérenchyme périphérique.

\section{V}

Les organes aériens sont tous pourvus d'éléments mécaniques périphériques. 


\section{INDEX BIBLIOGRAPHIQUE}

Haberlandt. - Uber die Grösse der Transpiration unserer Culturpflanzen. (Wiss. prakt. Unters. II, 1877.)

J. Sachs. - Ueber das Wachsthum der Haupt und Nebenwurzeln. (Arbeiten des bot. Instituts in Wür'zburg, 1873.)

de Bary. - Ueber die Wachsüberzüge des Epidermis.

(Botan. Zeitung, 1871.)

de Bary. - Vergleichende Anatomie, 1877.

Strasburger. - Das Botanische Prakticum, 1888.

G. Krauss. - Grundlinien zu einer Physiologie des Gerbstoffs. - Leipzig, 1889.

Baranelzky. - Epaississement des parois des éléments parenchymateux.(Ann. des sc. nat. $7^{\mathrm{e}}$ série. IV , 1886̉.)

Frimy el Urbain. - Etudes chimiques sur le squelette des végétaux. (Ann. des sc. nat. $6^{\mathrm{e}}$ série, XIII, 1882.)

Haberlandl. - Entwickelungsgeschichte des mechanischen Gewebesystems der Pflanzen. - Leipzig, 1879.

Schwendener. - Das mechanische Princip. - Leipzig, 1874.

Van Tieghem. - Deuxième mémoire sur les canaux sécréteurs des plantes. (Ann. des sc. nat. 7e série, I, 1885.)

Mlle A. Leblois. - Recherches sur l'origine et le développement des canaux sécréteurs el des poches sécrètrices. (Ann. des sc. nat. 7e sér. VI, 1887.)

Van Tieghium. - Recherches sur la symétrie de structure des plantes vasculaires. Ier mémoire : La Racine. (Ann. des sc. nat. 5e sér. XIII, 1871.) 
Russow. - Vergleichende Untersuchungen. (Mém. de l'Acad. des Sc. de St-Pétershourg, XIX, 1872.)

Van Tieghem. - Sur le réseau sus-endodermique de la racine. (Bulletin de la Socićté bot. XXXIV, 18887.)

- Sur le réseau de soutien de l'écorce de la racine. (Ann. des sc. nat. 7e sér. VII, 1888.)

- Sur les fibres libériennes primaires de la racine. (Ann. des Sc. nat. VIl, 1888.)

Costantin. - Recherches sur l'influence qu'exerce le milieu sur la structure des racines. (Ann. des Sc. nat. $7^{\text {e }}$ sér. 1, 1885.)

- Recherches sur la structure de la tige des plantes aquatiques. (Ann. des Sc. nat. 6e sér. XIX, 1884.)

Hugo v. Mohl. - Vermischte Schriften, 1845.

Kohl. - Anat. physiol. Untersuchung der Kallisalze und Kieselsälure in den Pflanzen. - Marburg, 1889.

Karellschikoff. - Ueber die faltenförmigen Verdickungen in den Zellen einiger Gramineen. (Bull. de la soc. imp. des naturalistes de Moscou. Tome XLI, 1868.)

Bolanische Zeilung. - Ueber das Kieselskelelt lebender Pflanzentheile, 1861, p. 226.

Schwendener. - Die Spaltöffnungen der Gramineen und Cypéraceen. (Sitzungsberichte dẹr kgl. Preuss. Akad. der Wiss. zu Berlin, 1889.

Volkens. - Die Flora der Aegyptisch-Arabischen Wüste. Berlin, 1887.

- Beziehungen zwischen Standort und anatomischen Bau der Vegetationsorgane. (Jahrb. d. Kgl. Gartens zu Berlin, B. IIJ, 1884.

Pfizer. - Beiträge zur Kenntniss der Hautgewebe. (Pringsheim's Jahrb. fur. w. Bot. VII.)

Tschirch. - Beiträge zù der Anatomie und dem Einrollungsmechanismus einiger Grasblätter. (Pringheim's Jahrb. f. w. Bol. XIII). 
Haberlandt. - Vergleichende Anatomie des Assimilatorischen Gewebesystems der Pflanzen, (Pringheim's Jahrb. XIII.)

Wigand. - Einige Sätze über die physiologische Bedeutung des Gerbstoffs und der Pflanzenfarben. (Bot. Leit. 1862).

Klingc. - Vergl. histol. Untersuchung der Gramineenund Cỵperaceen Wurzeln. (Mém. de l'Acad. Imp. des Sciences de St-Pétersburg. VIIe sér. XXVI.)

Duval-Jouve. - Histotaxie des feuilles de Graminées Ann. des Sc. nat. sér. 6, t. I.)

Stahl. - Pllanzen und Schnecken, Jena, 1888.

Jules Sachs. - Vorlesungen über Pflanzenphysiologie. 1887.

Laux. - Beitrag zur Kenntniss der Leitbündel im Rhizom Monocotyler Pflanzen.

Schwendener. - Die Schutzscheiden und ihre Verdickungen.

Reichenbach. - - Icones, VIII.

Tschirch. - Ueber Durchbrechungen der mechanischen Ringe zum Zwecke der Leitung der Assimilationsprodukte. (Berichte der deutsch. Bot. Gesellsch. Jahırg. 1884. B. II.) 


\section{EXPLICATION DES PLANCHES ${ }^{1}$}

Fig. 1. - Section transversale de la tige de C. divulsa (schéma) montrant la distribution du stéréoıne (partie noire) et celles des cellules aqueuses qui l'accompagnent ( $c a$ ).

Fig. 2 - Appareil de plissement de la même espèce. Gut. cuticule plissée. g. Gaine fibreuse de la nervure médiane.

Fig. 3. D. divulsa. Stomate vu de face, enclavé dans l'épiderne zigzagué.

Fig. 4. - C. virens. Section transversale de la lige montrant les faisceaux disposés sur un rang. $l$. lacune.

Fig. 5. - Endoderme du rhizome de C. divulsa, avec le tissu contigu du côté extérieur. - m. méats intercellulaires.

Fig. 6. - Même partie chez C. muricata.

Fig. 7 a b. - C. divulsa. Ecorce externe du rhizome. $p$. poncluations. - $b$. partie plus interne.

Fig. 8. - C. divulsa. Section transversale d'une racine. Le péricambium existe entre l'endoderme et le vaisseau primaire $v$. - $n$. parenchyme noueux.

Fing. 9. - Nodosités dans le parenchyme en voie de résorption.

Fig. 10. - C. muricala, Bifurcations intracellulaires. m. méats intercellulaires proprement dits.

1 Dans les figures schématisées, la couleur noire indique le stéréome: pour les autres, le bleu indique l'assise péricambiale el le jaune les vaisseaux primaires. 
Fig. 11. - Poil-crochet sur l'arêtede la feuille de C. vulpina.

Fig. 12. - Parenchyme cortical du rlizome de C. vulpina. $-m$. méats intercellulaires. $-p$ ' $p^{\prime \prime}$. poncluations rues de face et de còté. - $b$. bifurcation dans la membrane cellulaire.

Fig. 13. - C. rulpina. Endoderme de la racine. L'épaississement n'a lieu que sur la moitiè interne des éléments.

Fig. 14. - C. remota. Section transversale d'une feuille dans sa partie médiane. $-l$. lacunes allongées. $c a$. cellules aqueuses. - L'épiderme supérieur occupe le tiers environ de l'épaisseur totale.

Fig. 15. - Section transversale de la tige de C. paniculata. - f. faisceaux sur un rang. - $l$. lacunes.

Fig. 16. - C. paniculata. Aspect et formation des nodosités de la racine.

Fig. 17. - C. hirta. Stomates vu de faces (a.) - b. le même en section transversale.

Fig. 18. - C. Oederi. Stomates, section transversale. Les cellules annexes proéminent fortement rers l'intérieur.

Fig. 19. - C. provincialis. Stomate vu de face. L'ouverture en est cachée par les épaississements des parois des cellules épidermiques.

Fig. 20. - Le même en section transversale, montrant les cellules stomatiques au fond de la cheminée formée par les cellules annexes.

Fig. 21. - C. alba. Section transversale du stomate. c. épaississement des parois des cellules stornatiques. Fig. 22. - C. hordeistichos. Stomate. Section transversale. rig. 23. - C. riparia. Stomate. - a) En section transversale au fond du puits formé par les rellules annexes. b) de face, les parois des cellules épidermicues forment deux $\mathrm{V}$ s'ourrint au-dessus de la fente stomatique. $-c$ ) le mème vu de face. 
200

Fig. 24. - C. acuta. Stomates. - a) de face. Les cellules annexes présentent une excavation vis-à-vis de la fente stomatique. $-b$ ) en section transversale. Les cellules annexes font saillie en dehors. $-p$. papilles épidermiques.

Fig. 25. -- C. maxima. a. - Stomate vu de face accompagné de deux papilles $p p^{\prime}$. - b) le mème en séction Iransversale montrant les collules annexes qui dépassent les cellules stomatiques et deux papilles de l'épiderme $p p$ qui forment un puits profond $(a)$.

Fig. 26. - C. depauperata. Stomate superficiel. $-b$.

Une bifurcation de membranes.

Fig. 27. - C. baldensis. Stomate superficiel en section transversale. Lumens arrondiś - Voir fig. $27 \dot{b}$, un stomate de la tige, vu de face.

Fig. 28. - C. arida. Stomate vu de face renfermant deux grosses gouttes d'un corps huileux $h$.

Fig. 29, - G. stricta. a.) Stomate en section transversale. Les cellules annexes proéminent fortement au-dessus de l'épiderme en entourant les cellules stomatiques. - p.'papilles à parois épaissies. $-b$ ) Schéma montrant l'organisation du stomate. Les cellules annexes forment un sinus profond au-dessus de l'ouverture. - ép. bandes d'épaississement des parois.

Fig. 30. - C. paniculata. Stomate. a) en section transversale, montrant l'enfoncement des cellules stomatiques sous l'épiderme. - b) vu de face, les cellules annexes ont épaissi irrégulièrement leurs parois.

Fig 31. - Stomate de C. arenaria.

Fig. 32. - C. Buxbaumii. - a) sans papilles. - b) accompagné de papilles.

Fig. 33. - C. glauca. - a) en section transversale mon- 
trant le stomate au fond du puits formé par les parois épaissies des cellules épidermiques. - b) un jeune stomate où les cellules stomatiques ne sont pas encore recouvertes par les cellules voisines. c) le même à un àgee plus avancé.

Fig. 34. - Le mème stomate vu de face. Dessin formé par les cellules épidermiques. On aperçoit à peine l'ouverture stomatique.

Fig. 35. - C. ampullacea. a) Stomate en section Iransversale au fond de son puits. b) et c) autres dispositions de ces mêmes stomates. - $b$ ) vu de face.

Fig. 36. - C. vulgaris. - a) Stomate vu de face avec. deux goutles d'huile. - b) le même montrant son léger enfoncement au-dessous des cellules annexes. Fig 37. - C. distans. - Stomate en section transversale. Fig. 38. - C. dislans. Genèse des stomates.

1. Une des cellules de l'épiderme.

2. Celte cellule s'est divisée en deux cellules dont la plus petite donnera naissance aux stomates (s).

3. Les cellules voisines $a$ et $b$ ont continué de croitre pendant que $s$ est restée stationnaire.

4. La cellule $s$ se cloisonne longiludinalement.

5. Les cellules voisines latéralement séparent chacune un segment qui formera la cellule annexe.

6. Le stomate est complet, entouré de ses quatre cellules.

Fig. 39. - Schema des stomates.

39 a. - Une cellule stomatique avec sa cellule annexe. $a$. bande d'épaississement. $-l$. lumen de lá cellule stomatique. $-d$. paroi de la même cellule. 39 b. - Les mêmes lettres que pour $39 a$.

Fig. 40. - G. nigra. Stomate enfoncé sous les papilles. Fig. 41. - C. atrata. Stomate et épiderme inférieur. 
Fig. 42. - Papille sur la tige de C. Davalliana. - $s$. cellules fibreuses. En coupe longitud. fig. 44.

Fig. 43. - C. baldensis. Stomate, la feuille est pourvue de cellules grandes et incolores laissant entre leurs parois de grands méats (sinus pariétaux, bifurca(ions, entonnoirs.)

Fig. 44. - Voir fig. 42.

Fig. 45. - C. Davalliana. Section transversale d'une feuille. - $m m \mathrm{~m}$. Groupes fibreux.

Fig. 46. - C. Davalliana. Seclion transversale d'une racine.

- en. endoderme à éléments en étriers. - $v$. vaisseau primaire contigu à l'endoderme. - $n$. nodosités.

Fig. 47. - Nodosités de la même racine à un très fort grossissement. En $a$, on voit encore un vestige de paroi radiale.

Fig. 48. - C. chordorrhiza. Section transversale d'un stolon. - e cellules épidermiques massives. $-s$. sclèrenchyme.

Fig. 49. - Cellules épidermiques du stolon, vues de face (a.) - b. en coupe longitudinale.

Fig. 50. - Même espèce. Eléments endodermiques à lumen pyriforme (de la racine.)

Fig. 51. - Assise pilifère el sclérenchymateuse de la racine, C. chordorhiza. - s. sclèrenclýme.

Fig. 52. - C. Schrcberi. - Feuille. a. épiderme supérieur portant des papilles pyramidales $p .-b . c$. section longitudinale des deux épidermes, $b$. épiderme inférieur ; c. épiderme supérieur.

Fig. 53. - Porlion d'une section transversale de la lige du même Carex. Faisceaux sur un rang. - c. $a$. cellules aqueuses.

Fig. 54. -- C. Schreberi. - Région épidermique du rliizome. -- Cloisonnement tangentiel. - ep. épiderme. - o. Luile colorée par l'orcanette. 
Fig. 55. - Eléments endodermiques du rhizome du même Carex. Parois d'épaisseur presque égale sur tout le pourtour.

Fig. 56. -- C. Schreberi. - Racine. - r. commencement de séries radiales. - en. endoderme. - $v$. vaisseau primaire séparé par le péricambium.

lig. 57. - C. brizoïdes. Rlizome. Eléments de l'endoderme.

Fig. 58. - C. brizoïdes. Feüille. - b). épiderme de la page inférieure avec cônes siliceux (c). Coupe longitudinale.

Fig. 59. - C. brizoïdes. Tige. Epiderme ; section longitudinale.

Fig. 60. - C. arida. Distribution du stéréôme et des cellules aqueuses autour d'un faisceau foliaire.

Fig. 61. - C. leporina. Epidermes, stéréomes et cellules aqueuses.

Fig. 62. - C. leporina Tige. - Anastomose des gaines fibreuses à la pointe interne des faisceaux.

Fig. 63. - Mème espèce. - Coupe longitudinale de la tige montrant le tissu spongieux el l'épaisseur des parois extérieures de l'épiderme. - $s$. un stomate.

Fig. 64. -- Même espèce. Eléments endodermiques de la racine.

Fig. 65. - C. Buxbaumii. Feuille. Papilles épidermiques. Fig. 66. - C. Buxbaumii. Racine. Assise pilifère. Poils unicellulaires.

Fig. 67. - Même espèce. Section transrersale d'une racine montrant l'enduderme fortement épaissi sur la moitié interne, les vaisseaux primaires confinant directement à celte assise. - c cellules rondes en séries radiales. - $n$. filaments à nodosités.

Figg. 68. - C. multiflora. Racine. - Section transversale montrant un faisceau libérien formé d'éléments de 
grandeur sensiblement égale el un endoderme en $\mathrm{U}$ épaissi sculement sur sa moitié interne. $-68 . b$. Eléments endoderiniques du rhizome. 68. c. élćment endodermique de la racine, section longitudinale.

Fig. 69. - C. stricta. Parenchyme cortical du rlizome : ponctuations, bifurcations el méats.

Fig. 70. - C. acuta. Feuille. - a). Papilles sur la page inférieure. - $b$ ). les mêmes papilles vus de face dans l'épiderme zigzagué.

Fig. 71. - G. acula. Rhizome. - L'endoderme, composé d'éléments massifs, ponctués, s'appuie sur une zone péricambiale de cellules très épaissies.

Fig. 72. - Parenchyme corlical du rhizome dans la même espèce. $-p p^{\prime}$ ponctuations de face el de côté.

Fig. 73. - C. acuta. Racine. - a) . section transversale montrant l'épaississement énorme des éléments de l'endoderme et leurs ponctuations ramifiées. $-b$. cellules endodermiques en section longitudinale.

Fig. 74. - Carex montana. - Papilles de la tige.

Fig. 75. - C. montana. Rhizome. - Eléments de l'anneau sclérenchymateux en coupe longitudinale. $p p$. Ponctuations.

Fig. 76. - C. præcox. Feuille. Appareil de plissement ; les cellules sont ici brusquement différencièes. $l$. l. lacunes.

Fig. 77 a. - C. præcox. Papilles crochets en section longitudinale; épiderme de la page supérieure de la feuille. - $b$. éléments endodermiques du rhizome. Fig. 77 c. - Eléments du rhizonıe en section longitudinale. Fig. 78. - C. prsecox. Racine. Fraction de l'endoderme avec cellules péricambiales.

Fig. 79. - C. alpestris. Eléments endodermiques du rhizome. 


\section{5}

Fig. 80. - Mèrne espèce. Elément très épaissi de l'endoderme de la racine.

Fig. 81. - C. nigra. Rhizome. Anneau sclérenchymateux.

- ep. épiderme.

Fig. 82. - Mème espèce. Eléments endodermiques du rhizome.

Fig. 83. - Mème espèce. Eléments endodermiques de la racine.

Fig. 84. - C. atrata. Anneau sclérenchymateux du rhizome. $-c$. cônes siliceux.

Fig. 85. - Mème espèce. Section longitudinale d'une racine montrant le commencement des séries radiales de l'écorce, et l'aspect pyrilorme des cellules de l'endoderme.

Fig. 86. - C. ornithopoda. Feuille. - Section longitudinale de l'épiderme supérieur.

Fig. 87. - C. ornithopoda. Tige. Section longitudinale de l'épiderme. $-p$ papille. $-b$. section transversale; $p$. papille. $-l$. $l$. lacunes.

Fig. 88. - Mème espèce. Rhizome. Eléments de l'endoderme.

Fig. 89. - Carex alba. Epiderme inférieur de la feuille, bifurcations.

Fig. 90. - Même espèce. Rhizome. - Eléments endodermiques.

Fig. 91. - Mème espèce. Racine. Elèments endodermiques. $-a$. en section transversale. $-b$. en section longitudinale.

Fig. 92 a. - C. nitida. Tige, section transversale ; cellules tannifères.

Fig. 92 b. - Carex nitida. Racine. — Endoderme à parois d'ègale èpaisseur.

Fig. 93. - Mème espèce. Racine. Insertion d'une radicelle aux dépens des cellules rhizogènes du 
206

péricambium $p$, situées entre l'endoderme et le vaisseau primaire $v$. Comparez avec fig. 185.

Fig. 94. - C. stricta. Racine. Assise pililère et sclérenchyme.

Fig. 95. - Carex glauca. Feuille. Crochets de l’épiderme supérieur. Epiderme zig̨zagué.

Fig. 96. - Mème espèce. Feuille. Papilles de la page inférieure, vues de face.

Fig. 97. - Portion d'une paroi zigzaguée, d'une cellule du mème épiderne; fortement grossie.

Fig. 98. - Portion de l'épiderme supérieur d'une feuille de C. glauca, montrant les dépôts cireux entre les couches de cuticularisation (d).

Fig. 99. - Même espèce. Epiderme inférieur d'une feuille.

- $p$. papille. - c. cône siliceux. - c. $t$. cellules tannifères. - $s$. revêtement fibreux d'un faisceau.

Fig. 100. - C. glauca. Cônes isolés par un traitement à l'acide sulfurique concentré et l'acide chrômique.

Fig. 101. - Section transversale à travers un jeune rhizome de C. glauca, montrant les points de Caspary, sur la moitié interne des cellules de l'endoderme.

Fig. 102. - Stomate d'une feuille très jeune de C. glauca.

Les cellules stomatiques n'ont pas encore épaissi leurs parois.

Fig. 103. - Jeune feuille de C. glauca, montrant les premiers débuts de la differenciation du tissu fondamental. - $v$. cellules aqueuses. - $f$. faisceau dèjà constitué. - $f$ ' faisceau en voie de formation. $m$. groupe d'éléments fibreux. - $s$. jeune stomate. - e. e.e. premiers vestiges de l'appareil de plissement.

Fig. 104. - L'appareil de plissement complètement développé. - $p$. une ponctuation. - Revêtement fibreux du faisceau médian. 
Fig. 105. - Mềme espèce. Portion de l'épiderme supérieur d'une feuille montran! les dépôts cireux $(d)$, et l'arrangement symétrique des cellules tannifères. s. stéréôme.

Fig. 106. - Quelques papilles en boucles de l'épiderme inférieur de la feuille (C. glauca).

Fig. 107. - C. glauca. Anneau sclérenchymateux du rhizome. - $c$. cònes siliceux. - $s$. sclérenchyme.

Fig. 108. - Mème espèce. Section transversale d'une tige, montrant la disposition des faisceaux et la répartition des cellules tannifères.

Fig. 109. - C. maxima. Faisceau libéro-ligneux d'une feuille, $-v \cdot v^{\prime}$ gros vaisseaux. - c. a. cellules aqueuses, ici très nombreuses. - $p$. papille de l'épiderme inférieur. $-n$. liber composé normalement de tubes criblès et de cellules annexes plus petites. - $s$. revêtement fibreux du faisceau.

Fig. 110. - C. maxima. Feuille. Epiderme de la page inférieure.

Fig. 111. - Même espèce. Portion du. rhizome offrant deux faisceaux de structure différente (collatėrale et concentrique).

Fig. 112 . - Même espèce. Eléments endodermiques de la racine. Lumens pyriformes.

Fig. 113. - Les mèmes en section longitudinale et montrant les nombreuses ponctuations dont ils sont creusés.

Fig. 114 A. - Photographie microscopique représentant la section transversale d'une racine qui offre le type habituel en toile d’araignée (C. maxima). En s. à la périphérie se trouve l'anneau de sclérenchyrne ici très épais; en $b$, rayon formé par les cellules rondes arrangées en tiles radiales; en $a$, filaments portant les nodosités ; $c$, couches de cellules arrondies et persistantes. 
Fïg. 114 B. - C. arenaria. Rhizome à faisceaux concentriques. - $l$. lacunes. - $s l$. anıcau sclérenchymateux peu accentué.

Fig. 114. - a.b.c. d. e. f. Nodosités.

Fig. 115. - C. strigosa. Feuille. - Section transversale par l'appareil de plissement, dont les cellules sont graduellement différenciées. $-l$. lacunes. $-c t$. cellules tannifères.

Fig. 116. - Mème espéce. Eléments endodermiques de la racine.

Fig. 117. - C. distans. Feuille. Région de l'épidermc inférieur montrant de nombreux méats intercellu. laires ordinaires et en entonnoirs (e).

Fìg. 118. - Cellules sous-épidermiques de la même région; vues de face.

Fig. 119. - Méats et bifurcations dans la région sousépidermique supérieure de la même feuille.

Fig. 120. - Section transversale de la feuille de C. distans. Au centre un faisceau entouré d'une gaine de cellules aqueuses. - $l$. lacune. $-c l$. cellules tannifères.

Fig. 121. - C. distans. Section transversale de la tige montrant la disposition des faisceaux el la répartition des cellules tannifères (c. l.)

Fig. 122. - C. distans. - Un stomate vu de face.

Fig. 123. - Mème espèce. Eléments endodermiques du rhizome.

Fig. 124. - Mème espèce. Cellules tannifères dans le paren_chyme cortical du rhizome. - $a . a^{2}$, deux cellules qui ont perdu leur contenu par le fait de la coupe.

Fig. 125. - Même espèce. Ponctuations dans le parenchyme ligneux du cylindre central (rhizome.)

Fig. 126. - Eléments endodermiques de la racine (C. distans.)

Fig. 127. - C. distans. Fenille. -- Epiderme de la page 
inférieure montrant les différents stades de dévelop pement des stomates.

Fig. 128. - Cloisonnement d'une cellule épidermique en vue de la formation des papilles. (C. distans, fenille.)

Fig. 129. - C. dépauperata. Section d'une racine montrant un vaisseau primaire $v$ séparé de l'endorlerme par un péricambium double.

Fig. 130. - C. punctata. Feuille. Cellules tannifères dans le mésophylle, en coupe longitudinale.

Fig. 131. - G. punctata. Section transversale d'une feuille montrant la répartition des cellules tannifères au pourtour des lacunes $l$.

Fig. 132. - C. punctata. Section transversale d'une tige montrant la disposition des faisceaux et la répartition des cellules tannifères.

Fig. 133. - Eléments endodermiques du rhizorne (même espèce.)

Fig. 134. - Mème espèce. Rhizome, Méals et bifurcacations dans le parenchyme cortical.

Fig. 135. - C. Oederi. Eléments endodermiques rhizome.)

Fig. 136. - Même espèce. Rhizome. Méats et bifurcalions dans le parenchyme cortical.

Fig. 137. - Même espèce. Eléments endodermiques de la racine. L'épaississement n'a eu lieu que dans la moitié interne des cellules.

Fig. 138. - Même espèce. Nodosilés dans le lissu cortical moyen.

Fig. 139. - Section transversale il travers l'écorce du rhizome de C. punctata. - en. endoderme. $s$. groupe fibreux. - ép. épiderme.

Fig. 140. - Distribution du stéréome dans la feuille de C. Pseudo-Cyperus. - $l$. lacunes.

Fig. 141. - Appareil de plissement de la même feuille. 
Ses cellules sont à peine différenciées des autres cellules de l'épiderme.

Fig. 142. - Même espèce. Section transversale d'une feuille montrant les bifurcations $b$ dans le mésophylle. - ép. épiderme.

Fig. 113. - Mème espèce. Eléments endodermiques du rhizome. b. Aspect du parenchyme cortical vers l'endoderme.

Fig. 144. - Même espèce. Section transversale de la racine. Endoderme en forme de croissant.

Fig. 145. - C. riparia. Epiderme inférieur de la feuille avec un stomate enfoncé. - $b$. bifurcations. $p$, papilles.

Fig. 146. - Les mêmes papilles vues de face au milieu de l'épiderme zigzagué.

Fig. 147. - Voir fig. 150.

Fig. 148. - Appareil de plissement de la feuille de C. riparia. - $l$. lacunes. - $s$. revêtement fibreux du faisceau médian.

Fig. 149. - C. riparia. Eléments endodermiques en U (rhizome).

Fig. 150. - Nodosités dans le parenchyme cortical du rhizome (G: riparia.)

Fig. 151. - C. riparia. Eléments endodermiques de la racine.

Fig. 152. - C. paludosa. Tige. Papilles-crochets.

Fig. 153. - C. ampullacea. Feuille. Sections longitudinales des deux épidermes. - $b$. Section tangentielle.

Fig. 15í. - Mème espèce. - $a$. Nodosités du parenchyme cortical du rhizome. - b. Eléments endodermiques du rhizome (forme de croissant.)

lig. 155. - C. ampullacea. Racine. Cellule du parenchyme cortical en section longitudinale.

Fig. 156. - Même espèce. Section transversale d'une racine. 
Fig. 157. - G. vesicaria. Appareil de plisse i ent de la feuille.

Fig. 158. - Même espèce. Faisceau concentrique du rlizome avec phloëme normal.

Fig. 159. - Détails de ce phloëme quelque peu schématisés.

Fig. 160. - C. hirta. Section transver'sale d'une feulle montrant la disposition du stéréome et des cellules aqueuses $(e . t)$. - $l$. lacune.

Fig. 161. - Poils unicellulaires des feuilles (C. hirta.)

Fig. 162. - Carex arenaria. Section transversale d'une racine. Endoderme à parois d'égale épaisseur. $v$. vaisseau primaire séparé par le péricambium de l'assise endodermique.

Fig. 163. - C. baldensis. Portion d'une section transver'sale d'une feuille. Stéréome allongé en colonne. - c. a. cellules aqueuses.

Fig. 164. - Mêrne espèce. Eléments endodermiques du rhizome.

Fig 165. - Mêrne espèce. Rlıizorne. - Région collenchymateuse sous-épidermique. - $c$. cônes siliceux. - ép. épiderme épaissi.

Figg. 166. - Même espèce. Eléments endodermiques de la racine.

Fig. 167. - Même espèce. Une nodosilć du parenchyme cortical (racine).

Fig 168. - C. hirta. Faisceau libéro-ligneux de la feuille. Colonne de stéréome interrompue par les cellules ayueuses $c t$.

Fig. 169. - C. provincialis. Feuille.

a. - Epiderme supérieur, papille p. - c. a. cellules aqueuses. $-s$. stéréome. - $b$. papille en section longiludinale. - $s$. stéréome. - $a$ " vue de face. - c. dessins formés par les couches de 
cuticule dins les éléments de l'épiderme supérieur. - $d$. épaississement des parois de l'épiderme aux arètes de la feuille.

Fig. 170. - Nême espèce. Schéma montrant la disposition des cellules aqueuses el du stéréome dans la feuille. (c. a. cellules aqueuses).

Fig. 171. - Mème espèce. Phizome. - Endorlerme et poncluations dans le parenchyme cortical. - $a$. poncluations de face el de profil. - $b$. bifurcations.

Fig. 172. - Parenchyme cortical, section longitudinale. - a.) poncluations.

Fig. 173. - Nême espèce. a.) Pacine. Parenchyme liģneux, section longitudinale.

Figg 174. - Mème espèce. Rhizome. Méats dans le parenchyme du cylindre central, $s$, gaine fibreuse du faisceau $\%$.

Fig. 175. - C. hordéistichos. Feuille. Apppareil de plissement. Jointures collenchymateuses (c).

Fig. 176. - Cônes siliceux dans une coupe longitudinale de l'épiderme (feuille).

Fiğ. 177. - Même espèce, feuille. — Eléments d'une gaine fasciculaire.

Fig̣. 178. - Même espèce. Stéréome el cellules aqueuses de la feuille.

Fig. 179. - Mème espèce. Méats dans le mésophylle.

Fig. 180. - Même espèce. Rhizome. Eléments endodermiques.

Fiğ 181 - Même espèce. Section transversale d'une racine. Vaisseaux primaires tantôt contigus à l'endoderme, lantôt sćparés.

Fig. 182. - C. Grayi. Tige. - Partie supéricure. Distribution du stéréome.

Fig. 183, - Même tige. -- Partie inférieure, 
213

Fig. 184. - C. Grayï. Faisceaux collatéraux du rhizome (g f). - st. sclérenchyme périphérique. - end. endoderme.

Fig. 184 a. - Eléments endodermiques du rhizome.

Fig. 185. - Carex orsithopoda. Insertion d'une radicelle vis-à-vis d'un faisceau libérien $(l)-$.$v . v$. les deux vaisseaux primaires contigus à l'endoderme. $r$. la radicelle insérée entre les deux faisceaux $v v$.

Fig. 186. - 1. 2. 3. 4. Spécimens des faisceaux d'une racine; (4)C. Davalliana.

Dessiné el pholographié d'après nature.

A. $M$.

Genève, 1890. 

La Faculte des Sciences autorise limpression de la présente thèse, sans exprimer d'opinion sur les propositions qui s'y trouvent énoncées.
Le Doten de la Faculté,

G. OLTRAMARE.

Geneve, le ro Férier 1891. 



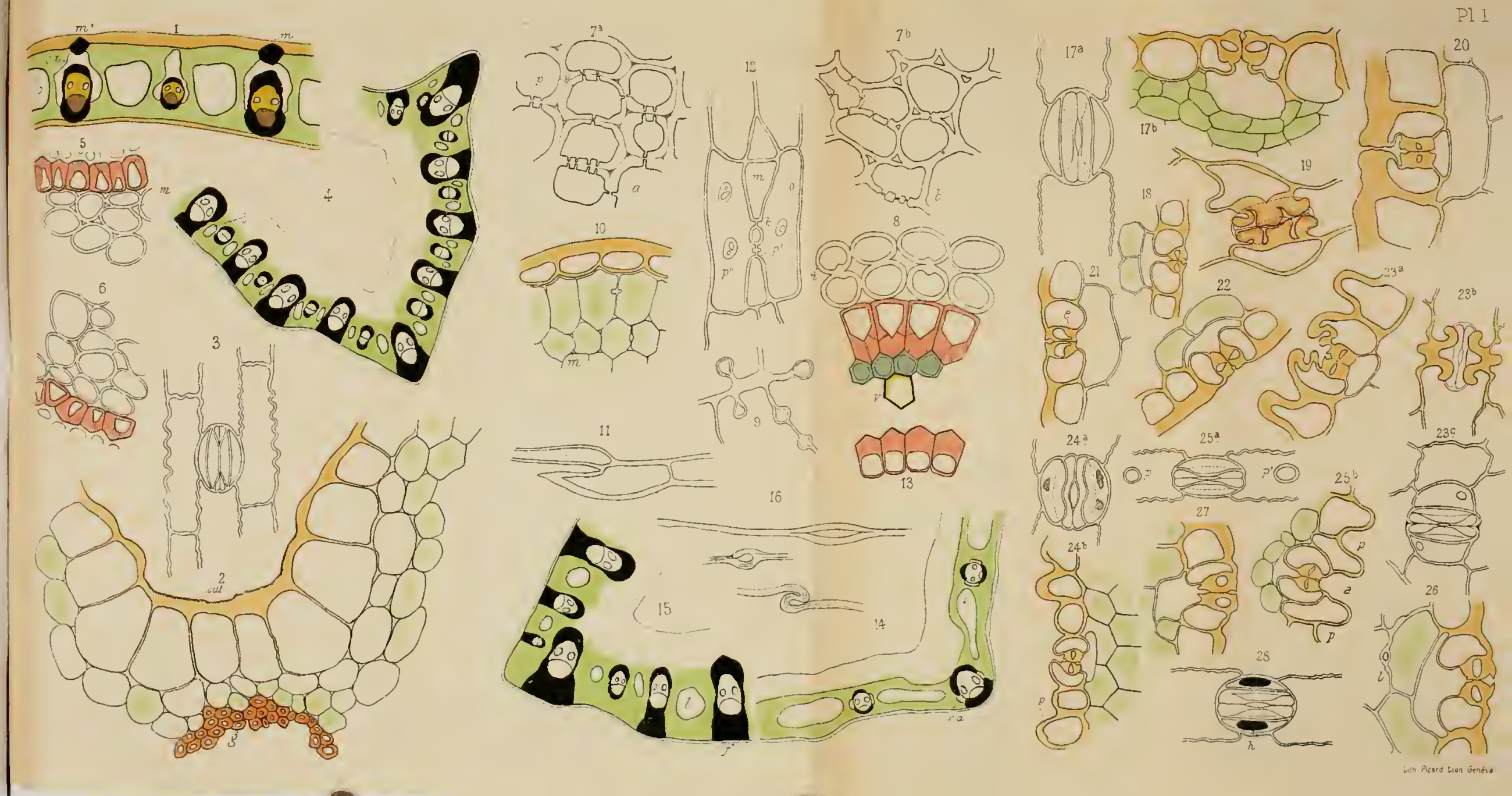




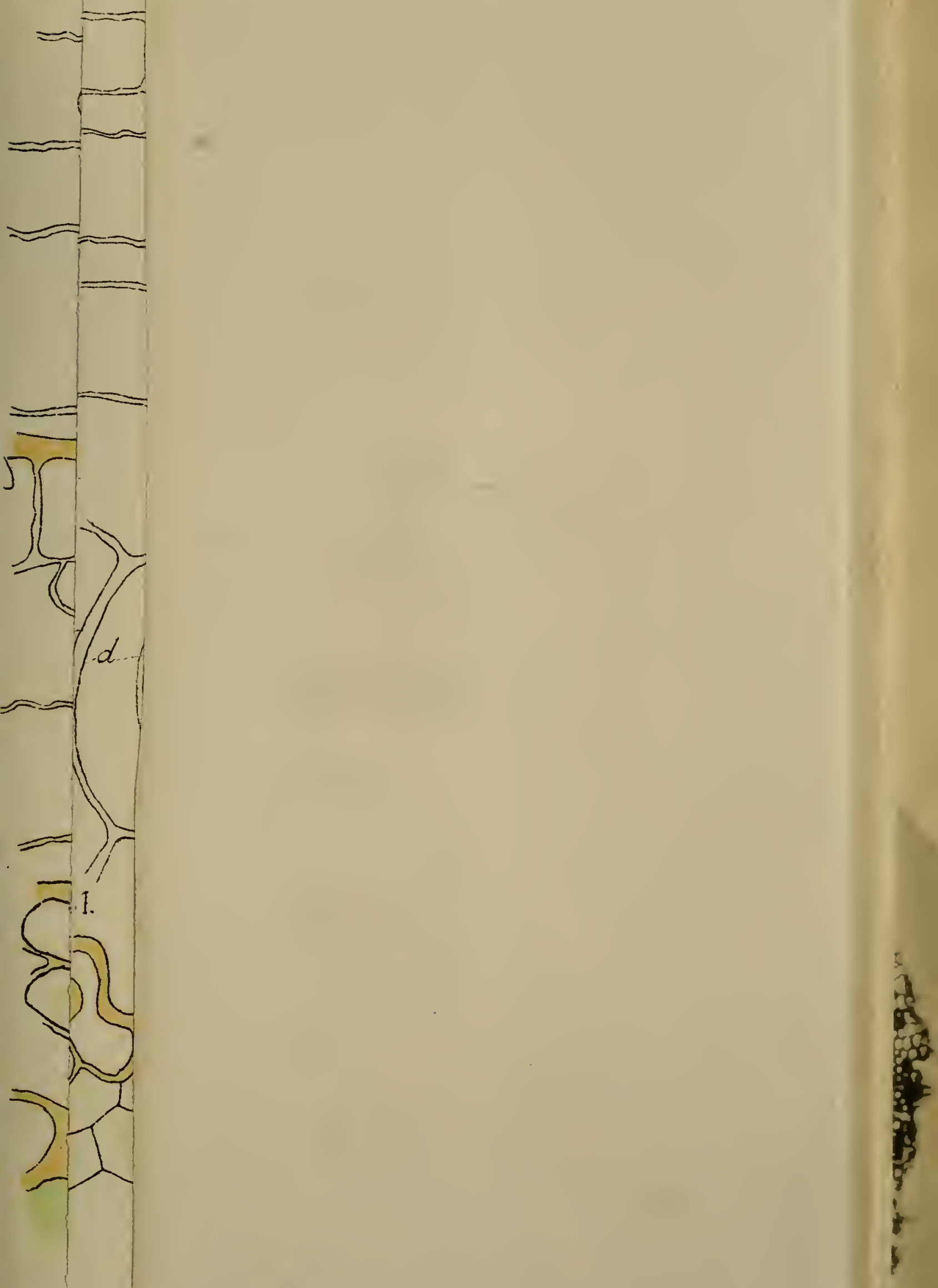




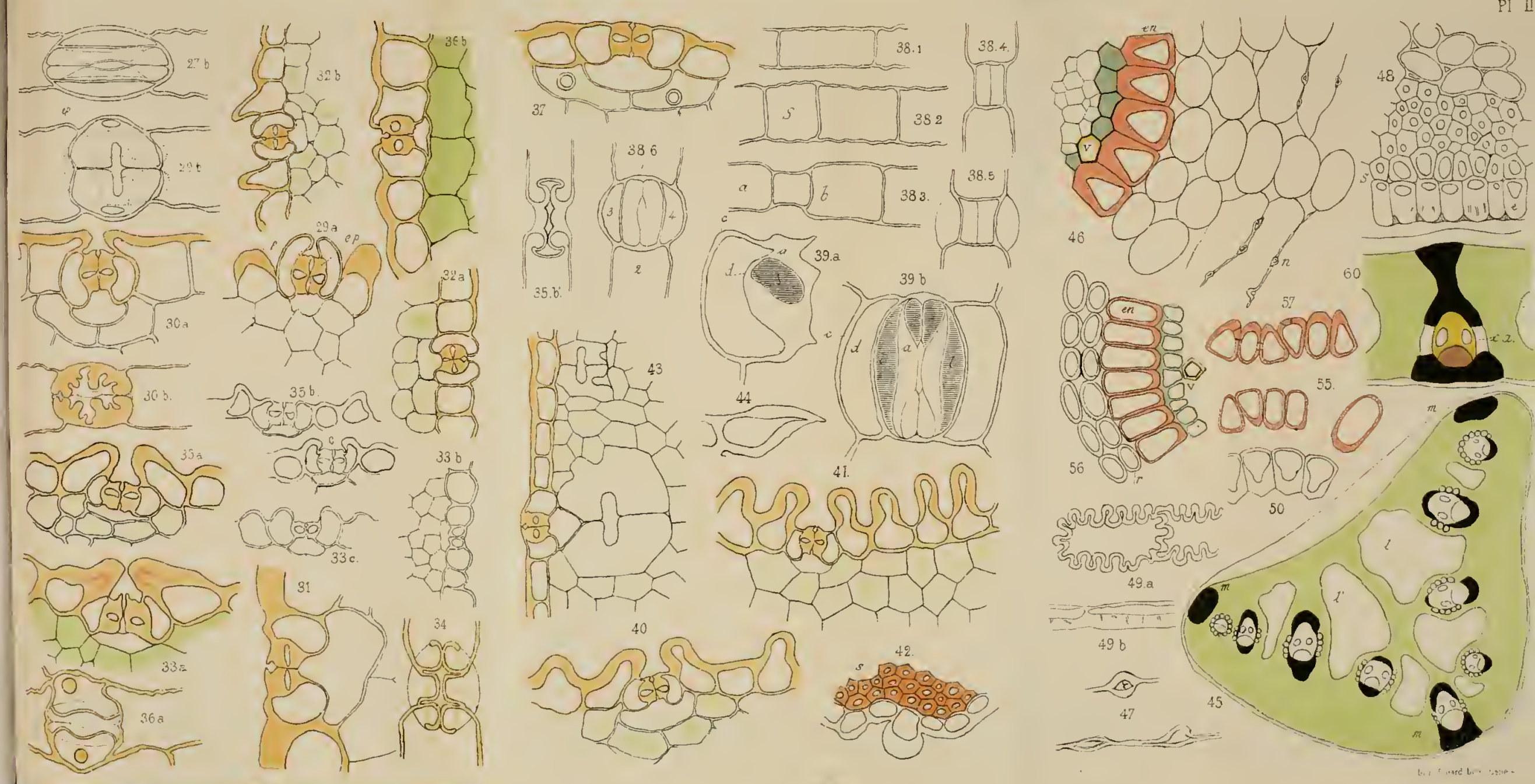









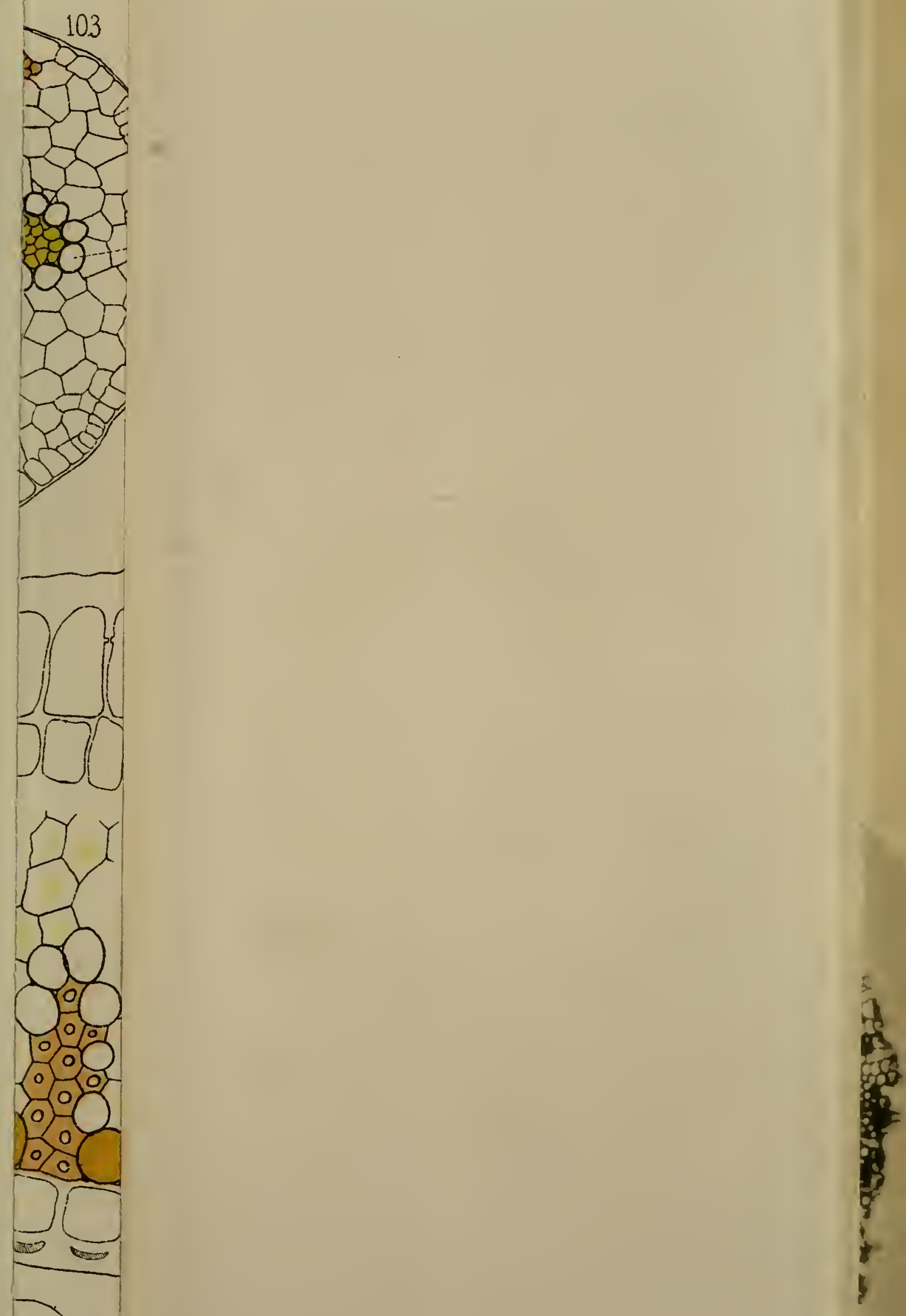


2 0 8.

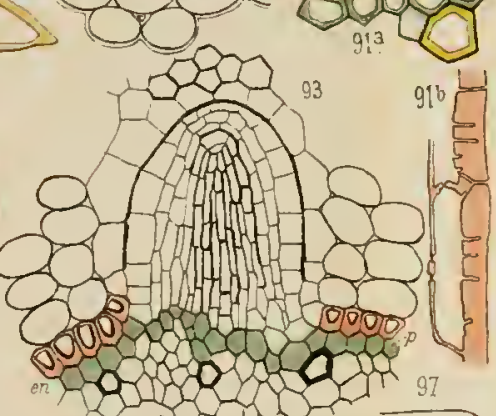

óç유os

(10)

(4)

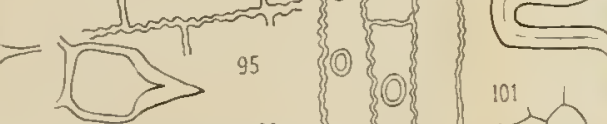

$\prod_{0 \rightarrow 00 \% 000}$

$a_{0} \theta$

(1) 000 \%
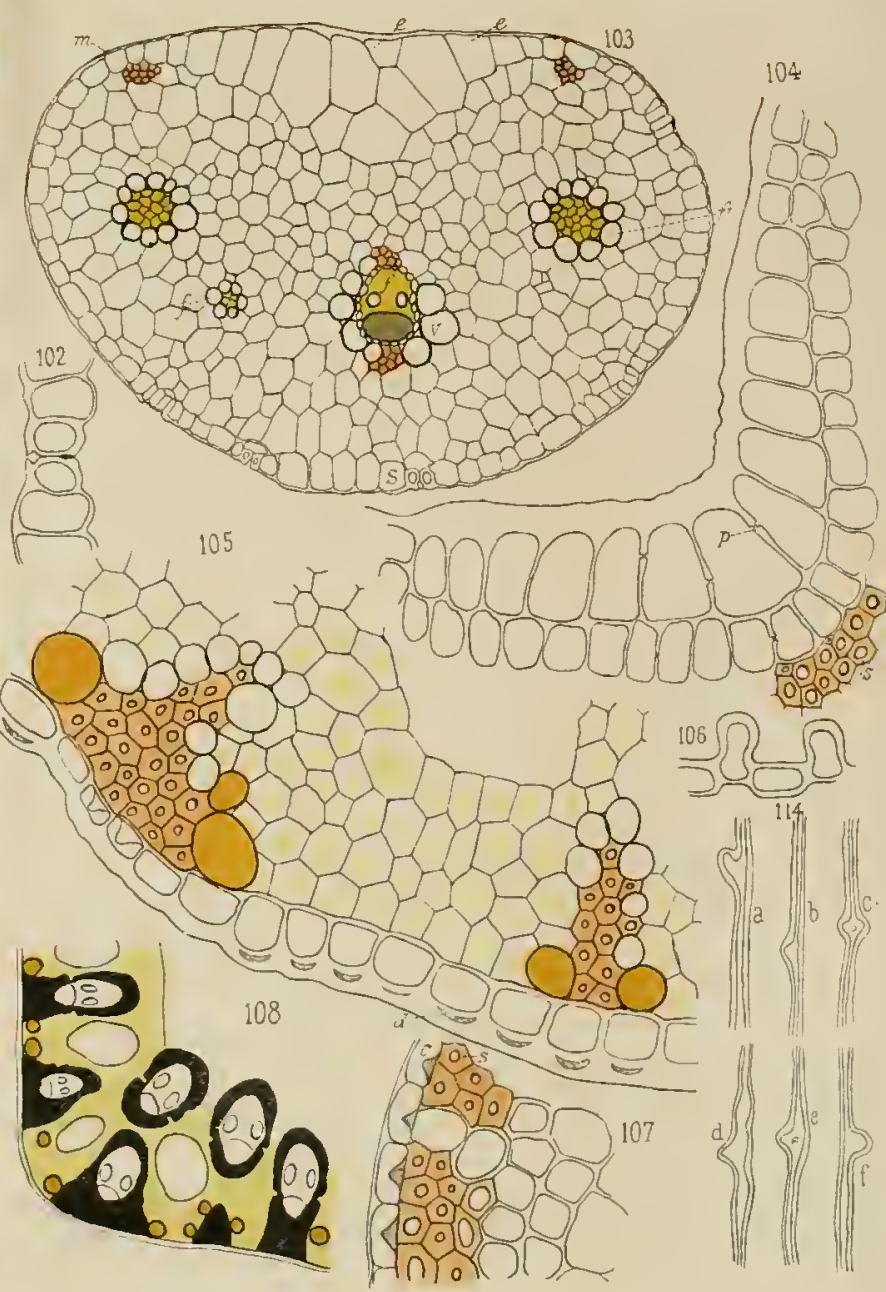

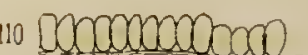
vn
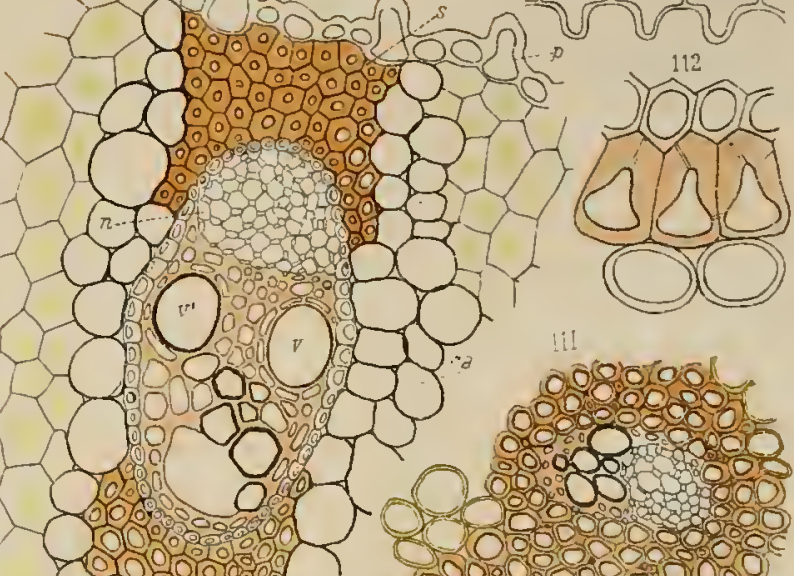

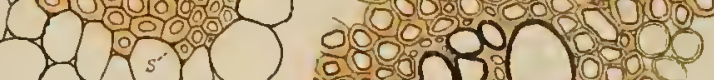
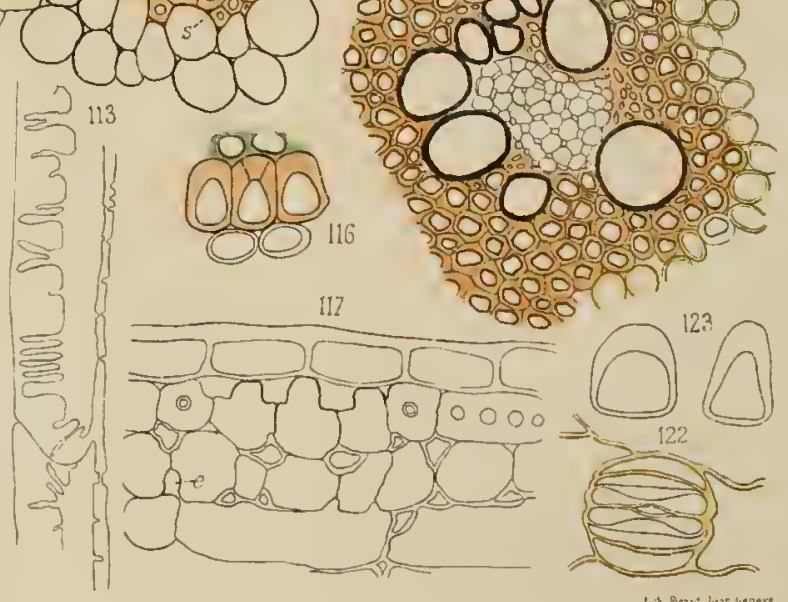

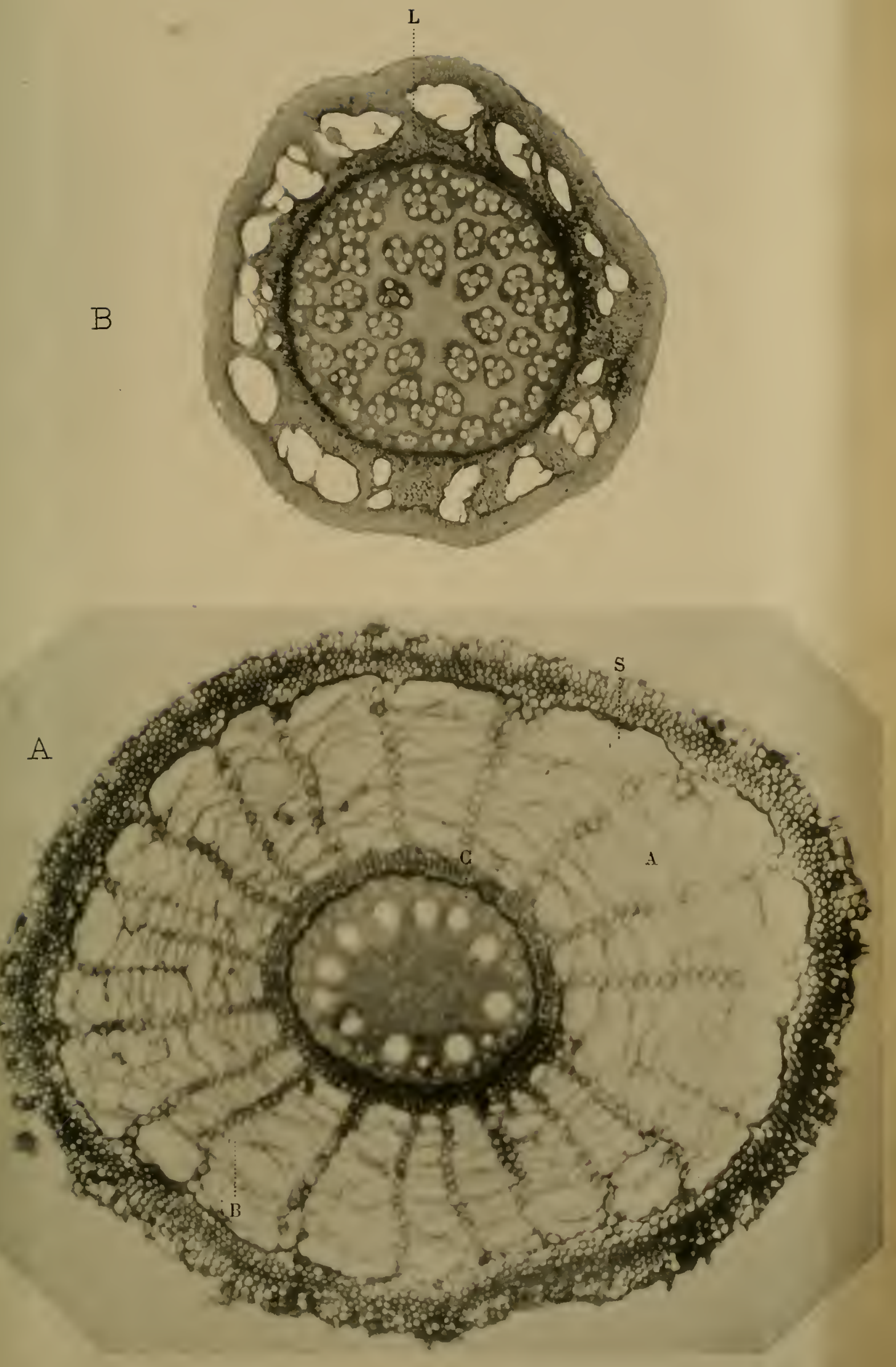



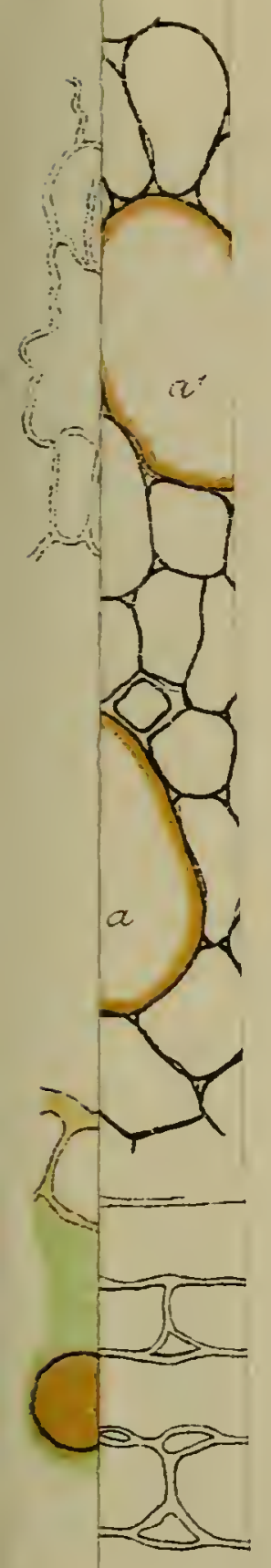




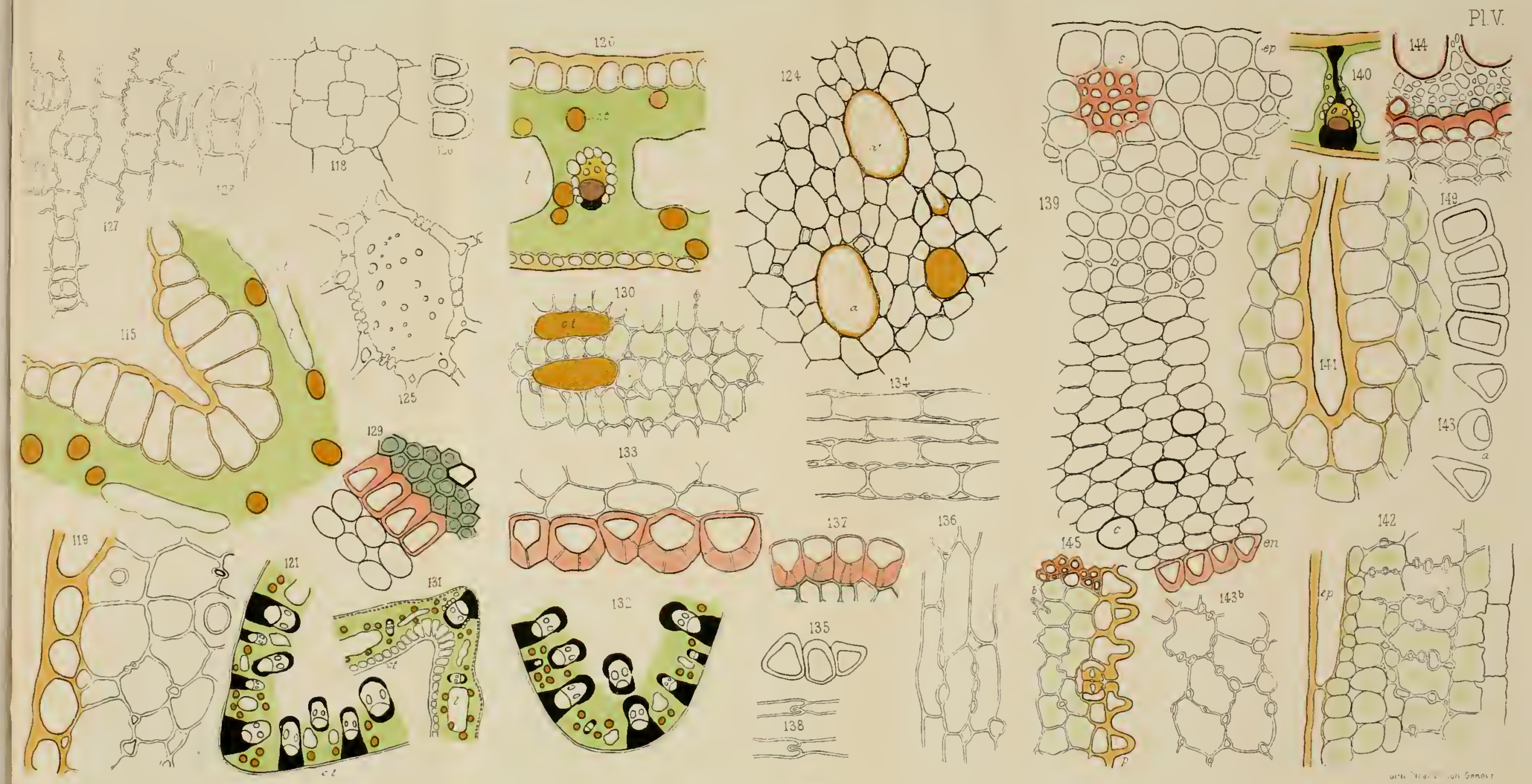



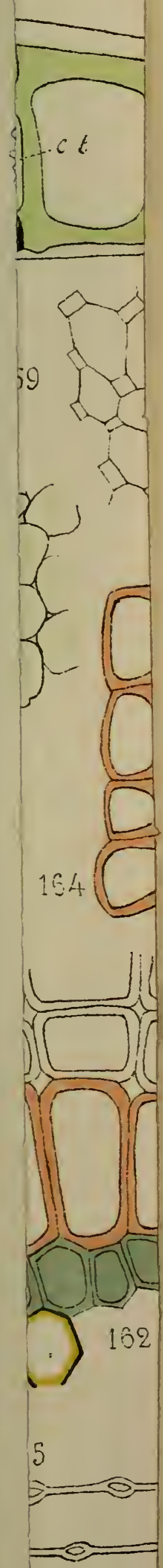

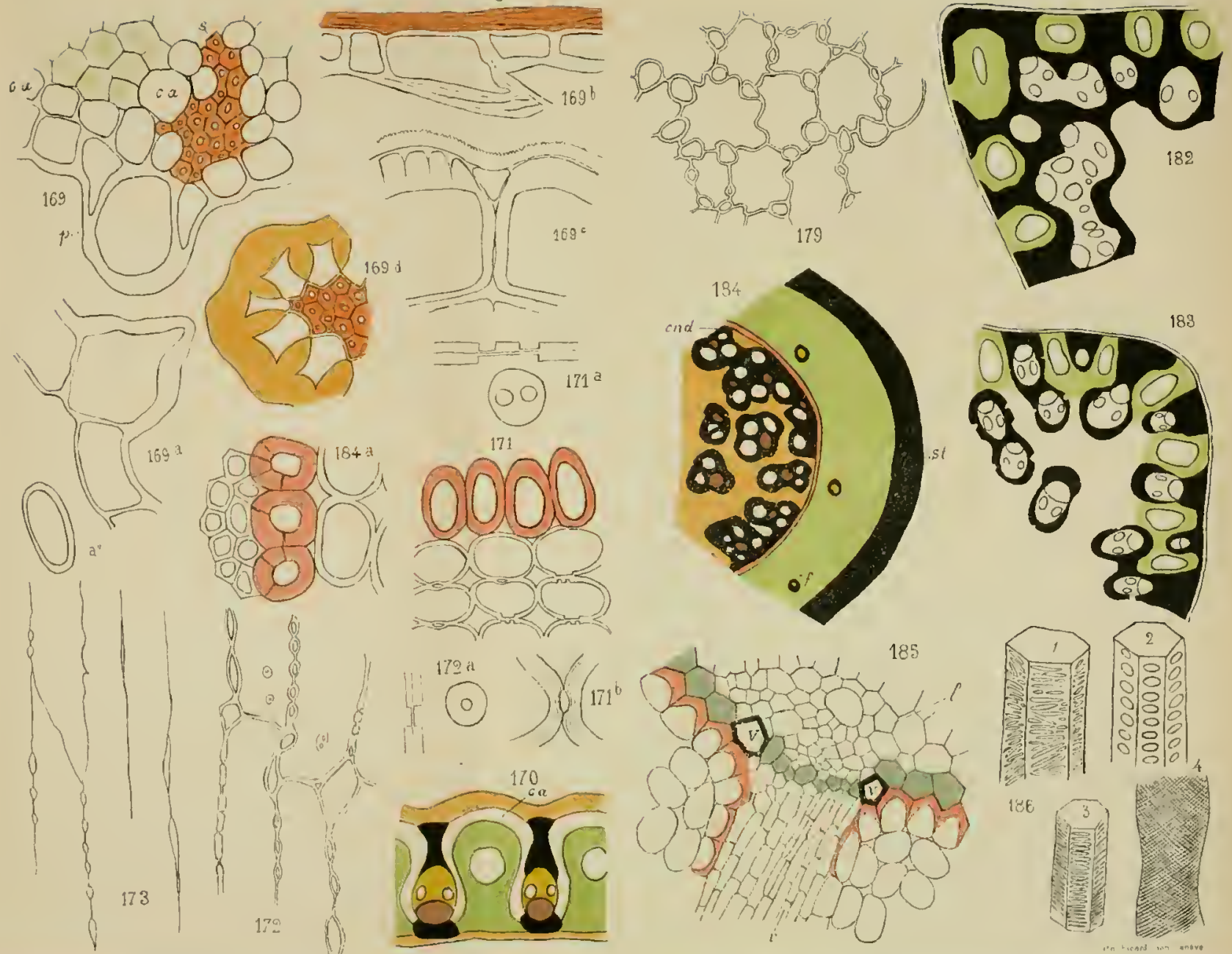


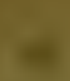

$-$

tats
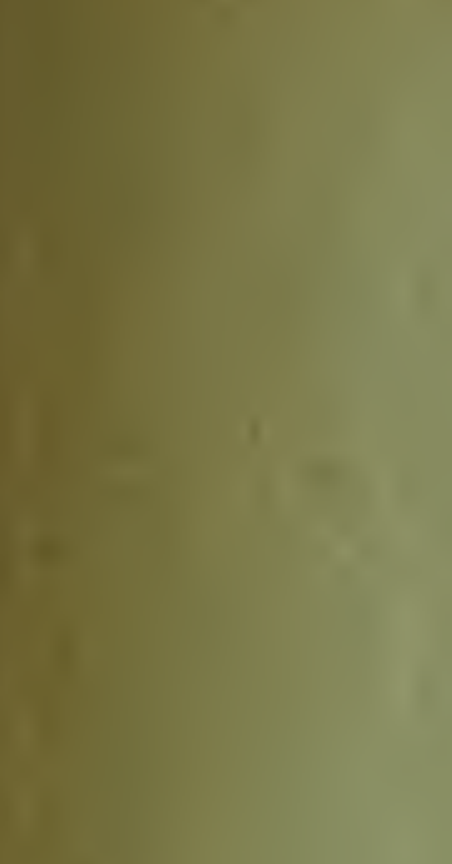

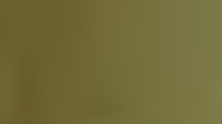

$\sqrt{2}+2+2$

1.

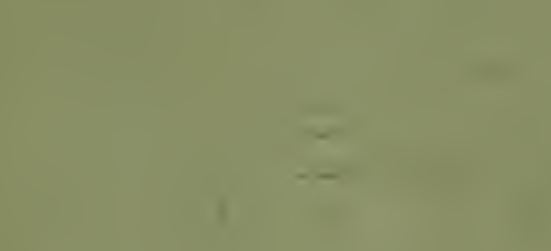

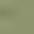

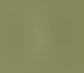

$-$

ters
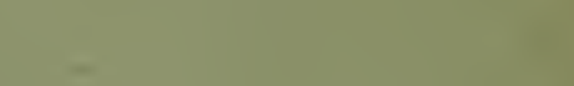

- +1

$-$

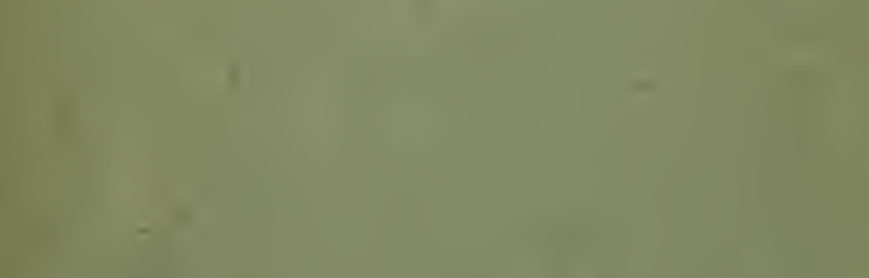

$$
\begin{aligned}
& -
\end{aligned}
$$$$
-
$$ 

